Supporting Information for

\title{
Oxetane intermediate during a direct aldol reaction: Stereoselective [5+1] annulation affording tetralines
}

Takeshi Kuri, Yoshihiko Mizukami, Mio Shimogaki, Morifumi Fujita*

Graduate School of Material Science, University of Hyogo, Kohto, Kamigori, Hyogo 678-1297, JAPAN

fuji@sci.u-hyogo.ac.jp

\section{Contents of the Supporting Information}

\section{Experimental Section}

Table S1. Effect of substituent of the substrate 3

Table S2. Crystal data and structure refinement for 4 al

Figure S1. An ORTEP view (50\% probability level) of 4 al

Scheme S1. Energy profile (in $\mathrm{kcal} / \mathrm{mol}$ ) for formation of trans tetraline product

Scheme S2. Comparison of energy profiles (in $\mathrm{kcal} / \mathrm{mol}$ ) for the trans tetraline formation and Grob elimination

Scheme S3. Energy profile (in kcal/mol) for Grob elimination

Scheme S4. Energy profile (in $\mathrm{kcal} / \mathrm{mol}$ ) for formation of cis tetraline product 


\section{Experimental Section}

General. Proton NMR, ${ }^{13} \mathrm{C}\left\{{ }^{1} \mathrm{H}\right\} \mathrm{NMR}$, and ${ }^{13} \mathrm{C}\left\{{ }^{1} \mathrm{H},{ }^{19} \mathrm{~F}\right\}$ NMR spectra were measured on a JEOL JNM-ECZ600R spectrometer as solutions in $\mathrm{CDCl}_{3}$. Proton NMR spectra were recorded using the residual $\mathrm{CHCl}_{3}$ as an internal reference (7.24 ppm) and ${ }^{13} \mathrm{C}$ NMR using $\mathrm{CDCl}_{3}$ as an internal reference (77.00 ppm). For mass spectra measurements was used JEOL JMS-T100LC.

Material. Unless otherwise noted, the reagents were commercially available and were used without further purification. Dichloromethane was purified by distillation over calcium hydride. The

${ }^{18} \mathrm{O}$-labeled benzaldehyde was prepared by nucleophilic substitution of benzyl chloride with ${ }^{18} \mathrm{OH}_{2}$ and subsequent oxidation with Dess-Martin periodinane according to the reported procedures. ${ }^{\mathrm{S}}{ }^{\mathrm{O}}$

Preparation of Substrates 3a-e. Substrate 3b was prepared by the reaction of acetoacetate with phenethyl bromide according to the literature. ${ }^{\mathrm{S} 2}$ Substrates 3a and 3d were prepared by Grignard reaction of $N$-methoxy- $N$-methyl-4-arylbutanamide with phenylmagnesium bromide according to the literature. ${ }^{\mathrm{S} 3}$ Similarly, substrate 3e was prepared from 4-(4-(trifluoromethyl)phenyl)butanenitrile ${ }^{\mathrm{S} 4}$ with phenylmagnesium bromide. Selected data for $3 \mathrm{e}:{ }^{1} \mathrm{H}$ NMR $\left(600 \mathrm{MHz}, \mathrm{CDCl}_{3}\right) \delta 7.91(\mathrm{~d}, J=7.6$ $\mathrm{Hz}, 2 \mathrm{H}), 7.54(\mathrm{t}, J=7.6 \mathrm{~Hz}, 1 \mathrm{H}), 7.52(\mathrm{~d}, J=8.3 \mathrm{~Hz}, 2 \mathrm{H}), 7.44(\mathrm{t}, J=7.6 \mathrm{~Hz}, 2 \mathrm{H}), 7.30(\mathrm{~d}, J=8.3$ $\mathrm{Hz}, 2 \mathrm{H}$ ), $2.98(\mathrm{t}, J=7.6 \mathrm{~Hz}, 2 \mathrm{H}$ ), $2.76(\mathrm{t}, J=7.6 \mathrm{~Hz}, 2 \mathrm{H}$ ), 2.08 (quint, $J=7.6 \mathrm{~Hz}, 2 \mathrm{H}$ ); ${ }^{13} \mathrm{C}\left\{{ }^{1} \mathrm{H},{ }^{19} \mathrm{~F}\right\}$ NMR $\left(150 \mathrm{MHz}, \mathrm{CDCl}_{3}\right) \delta 199.7,145.8,136.9,133.1,128.8,128.6,128.0,125.3,125.3$, 37.4, 35.0, 25.3; HRMS (ESI-TOF) $\mathrm{m} / z$ calcd for $\mathrm{C}_{17} \mathrm{H}_{15} \mathrm{~F}_{3} \mathrm{NaO}(\mathrm{M}+\mathrm{Na})^{+} 315.0973$, found 315.0980 .

Substrate 3c was also prepared from $N$-methoxy- $N$-methyl-4-phenylbutanamide as follows. To a stirred solution of $N$-methyl imidazole $(1.23 \mathrm{~g}, 15 \mathrm{mmol})$ in THF $(25 \mathrm{~mL})$ at $-78{ }^{\circ} \mathrm{C}$ was added $\mathrm{n}$-BuLi (2.6 $\mathrm{M}$ in hexane, $5.2 \mathrm{~mL}, 14 \mathrm{mmol}$ ). After the mixture was refluxed for $30 \mathrm{~min}$, $N$-methoxy- $N$-methyl-4-phenylbutanamide $(1.04 \mathrm{~g}, 5 \mathrm{mmol})$ was added to the mixture at $-78{ }^{\circ} \mathrm{C}$. After the mixture was refluxed for $9 \mathrm{~h}$, the reaction mixture was then quenched by aqueous acetic acid, and extracted by ethyl acetate. The extracts were dried with $\mathrm{Na}_{2} \mathrm{SO}_{4}$, and concentrated in vacuo. The crude mixture was purified by $\mathrm{SiO}_{2}$ column (eluent: $5 \%$ ethyl acetate in hexane) to give $3 \mathbf{c}$ (1.09 g, 96\% yield) as an oil. Rf: 0.34 (10\% ethyl acetate in hexane), Selected data for $3 \mathrm{c}:{ }^{1} \mathrm{H}$ NMR (600 $\left.\mathrm{MHz}, \mathrm{CDCl}_{3}\right) \delta 7.24(\mathrm{t}, J=7.6 \mathrm{~Hz}, 2 \mathrm{H}), 7.19(\mathrm{~d}, J=7.6 \mathrm{~Hz}, 2 \mathrm{H}), 7.15(\mathrm{t}, J=7.6 \mathrm{~Hz}, 1 \mathrm{H}), 7.11$ (s, $1 \mathrm{H}), 6.99(\mathrm{~s}, 1 \mathrm{H}), 3.96(\mathrm{~s}, 3 \mathrm{H}), 3.14(\mathrm{t}, J=7.6 \mathrm{~Hz}, 2 \mathrm{H}), 2.69(\mathrm{t}, J=7.6 \mathrm{~Hz}, 2 \mathrm{H}), 2.03$ (quint, $J=7.6$

(S1) Du, C.; Wang, X.; Jin, S.; Shi, H.; Li, Y.; Pang, Y.; Liu, Y.; Cheng, M.; Guo, C.; Liu, Y. Asian J. Org. Chem. 2016, 5, 755.

(S2) Harikrishna, M.; Mohan, H. R.; Dubey, P. K.; Shankar, M.; Subbaraju, G. V. Synth. Commun. 2012, 42, 1288-1305.

(S3) Torres-Ochoa, R. O.; Wang, Q.; Zhu, J. Chem. Eur. J. 2019, 25, 9477-9484.

(S4) Shimogaki, M.; Fujita, M.; Sugimura, T. Angew. Chem. Int. Ed. 2016, 55, 15797-1580 
$\mathrm{Hz}, 2 \mathrm{H}) ;{ }^{13} \mathrm{C}\left\{{ }^{1} \mathrm{H}\right\} \mathrm{NMR}\left(150 \mathrm{MHz}, \mathrm{CDCl}_{3}\right) \delta 192.8,142.9,141.8,128.8,128.5,128.3,126.8,125.8$, 38.5, 36.2, 35.3, 25.8; HRMS (ESI-TOF) $\mathrm{m} / z$ calcd for $\mathrm{C}_{14} \mathrm{H}_{16} \mathrm{~N}_{2} \mathrm{NaO}(\mathrm{M}+\mathrm{Na})^{+} 251.1160$, found 251.1155 .

Table S1. Effect of substituent of the substrate 3<smiles>[X]c1ccc(CCCC(=O)c2ccccc2)cc1</smiles>

3

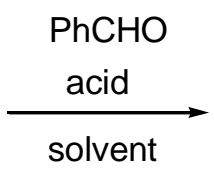

reflux<smiles>[X]c1ccc2c(c1)C(c1ccccc1)[C@H](C(=O)c1ccccc1)C[C@H]2C</smiles>

a: $\mathrm{X}=\mathrm{H}, \mathbf{d}: \mathrm{X}=\mathrm{CH}_{3}, \mathbf{e}: \mathrm{X}=\mathrm{CF}_{3}$

\begin{tabular}{lllcc}
\hline entry & substrate & \multicolumn{1}{c}{ acid } & time $/ \mathrm{h}$ & yield $/ \%$ \\
\hline 1 & $\mathbf{3 a}$ & $\mathrm{BF}_{3} \cdot \mathrm{OEt}_{2}$ & 2 & $89^{\mathrm{a}}$ \\
2 & $\mathbf{3 a}$ & $\mathrm{Me}_{3} \mathrm{SiOTf}_{1}$ & 2 & $88^{\mathrm{a}}$ \\
3 & $\mathbf{3 b}$ & $\mathrm{BF}_{3} \cdot \mathrm{OEt}_{2}$ & 1.5 & $94^{\mathrm{b}}$ \\
4 & $\mathbf{3 c}$ & $\mathrm{Me}_{3} \mathrm{SiOTf}^{\mathrm{c}}$ & 18 & $74^{\mathrm{b}}$ \\
\hline
\end{tabular}

${ }^{\mathrm{a}}$ The data has been described in Table $1 .{ }^{\mathrm{b}}$ The data has been described in Table 2.

\section{trans-(1,2,3,4-Tetrahydro-1-phenylnaphthalen-2-yl)(phenyl)methanone (4aa)}

To a stirred solution of $\mathbf{3 a}(112 \mathrm{mg}, 0.5 \mathrm{mmol})$ and benzaldehyde $(63 \mathrm{mg}$, $0.6 \mathrm{mmol})$ in a mixed solvent of hexane and dichloromethane $(9: 1(\mathrm{v} / \mathrm{v}), 2$ $\mathrm{mL})$ was added $\mathrm{BF}_{3} \cdot \mathrm{OEt}_{2} \quad(50 \mu \mathrm{L}, 0.4 \mathrm{mmol})$. The mixture was refluxed<smiles>O=C(c1ccccc1)[C@H]1CCc2ccccc2C1c1ccccc1</smiles>
with an oil bath for $2 \mathrm{~h}$. The reaction mixture was then quenched by $\mathrm{Na}_{2} \mathrm{CO}_{3}$ aqueous solution, and extracted by dichloromethane. The extracts were dried with $\mathrm{Na}_{2} \mathrm{SO}_{4}$, and concentrated in vacuo. The crude mixture was purified by column chromatography $\left(\mathrm{SiO}_{2}\right.$, eluent: $5 \%$ ethyl acetate in hexane) to give 4aa (180 mg, $0.44 \mathrm{mmol}, 88 \%$ yield); Rf: 0.41 (10\% ethyl acetate in hexane); ${ }^{1} \mathrm{H} \mathrm{NMR}\left(600 \mathrm{MHz}, \mathrm{CDCl}_{3}\right) \delta 7.73(\mathrm{~d}, J=7.6 \mathrm{~Hz}, 2 \mathrm{H}), 7.46(\mathrm{t}, J=7.6 \mathrm{~Hz}, 1 \mathrm{H}), 7.34(\mathrm{t}, J=$ $7.6 \mathrm{~Hz}, 2 \mathrm{H}), 7.18(\mathrm{t}, J=7.6 \mathrm{~Hz}, 2 \mathrm{H}), 7.14-7.09(\mathrm{~m}, 5 \mathrm{H}), 7.03(\mathrm{t}, J=7.6 \mathrm{~Hz}, 1 \mathrm{H}), 6.81(\mathrm{~d}, J=7.6 \mathrm{~Hz}$, $1 \mathrm{H}), 4.67(\mathrm{~d}, J=9.0 \mathrm{~Hz}, 1 \mathrm{H}), 3.91(\mathrm{td}, J=10.2,3.0 \mathrm{~Hz}, 1 \mathrm{H}), 3.07(\mathrm{ddd}, J=16.5,11.7,5.5 \mathrm{~Hz}, 1 \mathrm{H})$, $2.90(\mathrm{dt}, J=16.5,4.3 \mathrm{~Hz}, 1 \mathrm{H}), 2.15(\mathrm{~m}, 1 \mathrm{H}), 1.96(\mathrm{~m}, 1 \mathrm{H}) ;{ }^{13} \mathrm{C}\left\{{ }^{1} \mathrm{H}\right\} \mathrm{NMR}\left(150 \mathrm{MHz}, \mathrm{CDCl}_{3}\right) \delta$ 202.5, 145.4, 139.2, 136.7, 136.0, 132.8, 130.1, 129.3, 128.4, 128.4, 128.1, 126.3, 126.1, 125.8, 50.9, 47.2, 29.2, 26.9; HRMS (ESI-TOF) $\mathrm{m} / z$ calcd for $\mathrm{C}_{23} \mathrm{H}_{20} \mathrm{NaO}(\mathrm{M}+\mathrm{Na})^{+} 335.1412$, found 335.1409. 
cis-(1,2,3,4-Tetrahydro-1-phenylnaphthalen-2-yl)(phenyl)methanone (cis-4aa)

To a stirred solution of 3a (112 mg, $0.5 \mathrm{mmol})$ and benzaldehyde (63 mg, $0.6 \mathrm{mmol})$ in a mixed solvent of hexane and dichloromethane $(9: 1(\mathrm{v} / \mathrm{v}), 2$ $\mathrm{mL}$ ) was added $\mathrm{BF}_{3} \cdot \mathrm{OEt}_{2}(50 \mu \mathrm{L}, 0.4 \mathrm{mmol})$. The mixture was refluxed with an oil bath for $2 \mathrm{~h}$. The reaction mixture was then quenched by<smiles>O=C(c1ccccc1)C1CCc2ccccc2C1c1ccccc1</smiles>
$\mathrm{Na}_{2} \mathrm{CO}_{3}$ aqueous solution, and extracted by dichloromethane. The extracts were dried with $\mathrm{Na}_{2} \mathrm{SO}_{4}$, and concentrated in vacuo. The crude mixture containing 3aa as major trans-isomer and cis-isomer of 3aa $(9: 1)$ was purified by $\mathrm{SiO}_{2}$ column (eluent: $5 \%$ ethyl acetate in hexane) to elute 4aa. Continued column chromatography with $30 \%$ ethyl acetate in hexane as an eluent gave cis-4aa (15.5 mg, $9 \%$ yield). Rf: 0.41 (10\% ethyl acetate in hexane). The Rf value of cis-4aa is same as that of 4aa, but the successful separation of these isomers may be owing to difference in solubility in the eluent used. Selected data for $c i s-4 a a:{ }^{1} \mathrm{H}$ NMR $\left(600 \mathrm{MHz}, \mathrm{CDCl}_{3}\right) \delta 7.95(\mathrm{~d}, J=7.6 \mathrm{~Hz}, 2 \mathrm{H}), 7.61$ (t, $J=7.6 \mathrm{~Hz}, 1 \mathrm{H}), 7.51(\mathrm{t}, J=7.6 \mathrm{~Hz}, 2 \mathrm{H}), 7.23(\mathrm{~d}, J=7.6 \mathrm{~Hz}, 1 \mathrm{H}), 7.18(\mathrm{t}, J=7.6 \mathrm{~Hz}, 1 \mathrm{H})$, 7.09-7.05 (m, 4H), $6.90(\mathrm{~d}, J=7.5 \mathrm{~Hz}, 1 \mathrm{H}), 6.56(\mathrm{~d}, J=7.6 \mathrm{~Hz}, 2 \mathrm{H}), 4.68(\mathrm{~d}, J=5.5 \mathrm{~Hz}, 1 \mathrm{H}), 4.01$ (ddd, $J=12.4,5.5,2.7 \mathrm{~Hz}, 1 \mathrm{H}), 3.13(\mathrm{dd}, J=17.2,5.5 \mathrm{~Hz}, 1 \mathrm{H}), 2.99(\mathrm{ddd}, J=18.6,12.4,6.2 \mathrm{~Hz}$, 1H), $2.20(\mathrm{~m}, 1 \mathrm{H}), 1.93(\mathrm{~m}, 1 \mathrm{H}) ;{ }^{13} \mathrm{C}\left\{{ }^{1} \mathrm{H}\right\} \mathrm{NMR}\left(150 \mathrm{MHz}, \mathrm{CDCl}_{3}\right) \delta 201.3,141.8,138.2,137.2$, 136.5, 132.9, 130.5, 129.6, 129.0, 128.9, 128.4, 127.6, 126.7, 126.6, 126.1, 47.8, 47.4, 28.5, 19.0; HRMS (ESI-TOF) $m / z$ calcd for $\mathrm{C}_{23} \mathrm{H}_{20} \mathrm{NaO}(\mathrm{M}+\mathrm{Na})^{+} 335.1412$, found 335.1411.

\section{(1-(4-Bromophenyl)-1,2,3,4-tetrahydronaphthalen-2-yl)(phenyl)methanone (4ab).}

To a stirred solution of $\mathbf{3 a}(56 \mathrm{mg}, 0.25 \mathrm{mmol})$ and 4-bromobenzaldehyde (56 $\mathrm{mg}, 0.3 \mathrm{mmol})$ in a mixed solvent of hexane and dichloromethane $(9: 1(\mathrm{v} / \mathrm{v})$, $1 \mathrm{~mL}$ ) was added $\mathrm{BF}_{3} \cdot \mathrm{OEt}_{2}(25 \mu \mathrm{L}, 0.2 \mathrm{mmol})$. The mixture was refluxed with an oil bath for $2 \mathrm{~h}$. The reaction mixture was then quenched by $\mathrm{Na}_{2} \mathrm{CO}_{3}$ aqueous solution, and extracted by dichloromethane. The extracts were dried with $\mathrm{Na}_{2} \mathrm{SO}_{4}$, and concentrated in vacuo. The crude mixture was purified by column chromatography $\left(\mathrm{SiO}_{2}\right.$, eluent: $5 \%$ ethyl acetate in hexane) to give<smiles>O=C(c1ccccc1)[C@H]1CCc2ccccc2C1c1ccc(Br)cc1</smiles>
4ab (70.4 mg, $0.18 \mathrm{mmol}, 72 \%$ yield); Rf: 0.46 (10\% ethyl acetate in hexane); ${ }^{1} \mathrm{H}$ NMR (600 MHz, $\left.\mathrm{CDCl}_{3}\right) \delta 7.75(\mathrm{~d}, J=7.6 \mathrm{~Hz}, 2 \mathrm{H}), 7.49(\mathrm{t}, J=7.6 \mathrm{~Hz}, 1 \mathrm{H}), 7.37(\mathrm{t}, J=7.6 \mathrm{~Hz}, 2 \mathrm{H}), 7.30(\mathrm{~d}, J=8.3$ $\mathrm{Hz}, 2 \mathrm{H}), 7.14-7.10(\mathrm{~m}, 2 \mathrm{H}), 7.05-7.02(\mathrm{~m}, 3 \mathrm{H}), 6.77(\mathrm{~d}, J=7.6 \mathrm{~Hz}, 1 \mathrm{H}), 4.66(\mathrm{~d}, J=9.6 \mathrm{~Hz}, 1 \mathrm{H})$, $3.85(\mathrm{td}, J=10.3,2.3 \mathrm{~Hz}, 1 \mathrm{H}), 3.07(\mathrm{~m}, 1 \mathrm{H}), 2.91(\mathrm{dt}, J=16.5,4.1 \mathrm{~Hz}, 1 \mathrm{H}), 2.16(\mathrm{~m}, 1 \mathrm{H}), 1.93(\mathrm{~m}$, $1 \mathrm{H}) ;{ }^{13} \mathrm{C}\left\{{ }^{1} \mathrm{H}\right\} \mathrm{NMR}\left(150 \mathrm{MHz}, \mathrm{CDCl}_{3}\right) \delta 201.9,144.5,138.6,136.3,135.9,133.0,131.4,131.0$, 130.0, 128.6, 128.1, 126.1, 126.0, 120.2, 50.8, 46.5, 29.2, 27.1; HRMS (ESI-TOF) $\mathrm{m} / \mathrm{z}$ calcd for $\mathrm{C}_{23} \mathrm{H}_{19} \mathrm{BrNaO}(\mathrm{M}+\mathrm{Na})^{+} 413.0517$, found 413.0513. 


\section{1-(4-(Trifluoromethyl)phenyl)-1,2,3,4-tetrahydronaphthalen-2-yl)(phenyl)methanone (4ac).}

To a stirred solution of $\mathbf{3 a}(112 \mathrm{mg}, 0.5 \mathrm{mmol})$ and 4-trifluorobenzaldehyde $(0.13 \mathrm{~mL}, 1.0 \mathrm{mmol})$ in a mixed solvent of hexane and dichloromethane $(9: 1$ (v/v), $2 \mathrm{~mL}$ ) was added $\mathrm{BF}_{3} \cdot \mathrm{OEt}_{2}(180 \mu \mathrm{L}, 1.4 \mathrm{mmol})$. The mixture was refluxed with an oil bath for $1 \mathrm{~h}$. The reaction mixture was then quenched by $\mathrm{Na}_{2} \mathrm{CO}_{3}$ aqueous solution, and extracted by dichloromethane. The extracts were dried with $\mathrm{Na}_{2} \mathrm{SO}_{4}$, and concentrated in vacuo. The crude mixture was purified<smiles>O=C(c1ccccc1)[C@H]1CCc2ccccc2C1c1ccc(C(F)(F)F)cc1</smiles>
by column chromatography ( $\mathrm{SiO}_{2}$, eluent: $5 \%$ ethyl acetate in hexane) to give 4ac (177.2 mg, 0.47 mmol. 93\% yield); Rf: 0.5 (10\% ethyl acetate in hexane); ${ }^{1} \mathrm{H}$ NMR (600 MHz, $\left.\mathrm{CDCl}_{3}\right) \delta 7.74(\mathrm{~d}, J=7.6 \mathrm{~Hz}, 2 \mathrm{H}), 7.49(\mathrm{t}, J=7.6 \mathrm{~Hz}, 1 \mathrm{H}), 7.44(\mathrm{~d}, J=8.3 \mathrm{~Hz}, 2 \mathrm{H}), 7.36(\mathrm{t}, J=7.6$ $\mathrm{Hz}, 2 \mathrm{H}), 7.26(\mathrm{~d}, J=8.3 \mathrm{~Hz}, 2 \mathrm{H}), 7.16-7.12(\mathrm{~m}, 2 \mathrm{H}), 7.04(\mathrm{t}, J=7.6 \mathrm{~Hz}, 1 \mathrm{H}), 6.73(\mathrm{~d}, J=7.6 \mathrm{~Hz}$, $1 \mathrm{H}), 4.77(\mathrm{~d}, \mathrm{~J}=9.6 \mathrm{~Hz}, 1 \mathrm{H}), 3.88(\mathrm{~m}, 1 \mathrm{H}), 3.10(\mathrm{ddd}, J=16.5,11.0,4.8, \mathrm{~Hz}, 1 \mathrm{H}), 2.93(\mathrm{dt}, J=16.5$, $4.1 \mathrm{~Hz}, 1 \mathrm{H}), 2.19(\mathrm{~m}, 1 \mathrm{H}), 1.95(\mathrm{~m}, 1 \mathrm{H}) ;{ }^{13} \mathrm{C}\left\{{ }^{1} \mathrm{H},{ }^{19} \mathrm{~F}\right\} \mathrm{NMR}\left(150 \mathrm{MHz}, \mathrm{CDCl}_{3}\right) \delta$ 201.8, 149.7, 138.3, 136.3, 136.0, 133.1, 130.0, 129.7, 128.7, 128.6, 128.1, 126.3, 126.2, 125.4, 124.2, 50.8, 46.9, 29.3, 27.1; HRMS (ESI-TOF) $\mathrm{m} / z$ calcd for $\mathrm{C}_{24} \mathrm{H}_{19} \mathrm{~F}_{3} \mathrm{NaO}(\mathrm{M}+\mathrm{Na})^{+} 403.1286$, found 403.1284 .

\section{(1,2,3,4-Tetrahydro-1-(4-nitrophenyl)naphthalen-2-yl)(phenyl)meth} anone (4ad).

To a stirred solution of $\mathbf{3 a}$ ( $56 \mathrm{mg}, 0.25 \mathrm{mmol})$ and 4-nitroaldehyde (45 $\mathrm{mg}, 0.3 \mathrm{mmol})$ in a mixed solvent of hexane and dichloromethane (9 : $1(\mathrm{v} / \mathrm{v}), 1 \mathrm{~mL})$ was added $\mathrm{BF}_{3} \cdot \mathrm{OEt}_{2}(25 \mu \mathrm{L}, 0.2 \mathrm{mmol})$. The mixture was refluxed with an oil bath for $2 \mathrm{~h}$. The reaction mixture was then quenched by $\mathrm{Na}_{2} \mathrm{CO}_{3}$ aqueous solution, and extracted by<smiles>O=C(c1ccccc1)[C@H]1CCc2ccccc2C1c1ccc([N+](=O)[O-])cc1</smiles>
dichloromethane. The extracts were dried with $\mathrm{Na}_{2} \mathrm{SO}_{4}$, and concentrated in vacuo. The crude mixture purified by column chromatography ( $\mathrm{SiO}_{2}$, eluent: $5 \%$ ethyl acetate in hexane) to give 4ad (72.5 mg, $0.20 \mathrm{mmol}, 81 \%$ yield); Rf: 0.34 (10\% ethyl acetate in hexane); ${ }^{1} \mathrm{H}$ NMR (600 MHz, $\left.\mathrm{CDCl}_{3}\right) \delta 8.05(\mathrm{~d}, J=8.2 \mathrm{~Hz}, 2 \mathrm{H}), 7.76(\mathrm{~d}, J=7.6 \mathrm{~Hz}, 2 \mathrm{H}), 7.50(\mathrm{t}, J=7.6 \mathrm{~Hz}, 1 \mathrm{H}), 7.38(\mathrm{t}, J=7.6$ $\mathrm{Hz}, 2 \mathrm{H}), 7.33(\mathrm{~d}, J=8.2 \mathrm{~Hz}, 2 \mathrm{H}), 7.18-7.13(\mathrm{~m}, 2 \mathrm{H}), 7.05$ (t, $J=7.6 \mathrm{~Hz}, 1 \mathrm{H}), 6.69$ (d, $J=7.6 \mathrm{~Hz}$, $1 \mathrm{H}), 4.85(\mathrm{~d}, J=9.6 \mathrm{~Hz}, 1 \mathrm{H}), 3.88(\mathrm{~m}, 1 \mathrm{H}), 3.12(\mathrm{ddd}, J=16.5,11.7,5.5 \mathrm{~Hz}, 1 \mathrm{H}), 2.95(\mathrm{dt}, J=16.5$, $4.1 \mathrm{~Hz}, 1 \mathrm{H}), 2.22(\mathrm{~m}, 1 \mathrm{H}), 1.95(\mathrm{~m}, 1 \mathrm{H}) ;{ }^{13} \mathrm{C}\left\{{ }^{1} \mathrm{H}\right\} \mathrm{NMR}\left(150 \mathrm{MHz}, \mathrm{CDCl}_{3}\right) \delta 201.3,153.3,146.5$, 137.7, 136.0, 136.0, 133.3, 130.3, 129.9, 128.9, 128.7, 128.1, 126.5, 126.4, 123.7, 50.8, 46.8, 29.3, 27.2; HRMS (ESI-TOF) $\mathrm{m} / z$ calcd for $\mathrm{C}_{23} \mathrm{H}_{19} \mathrm{NNaO}_{3}(\mathrm{M}+\mathrm{Na})^{+} 380.1263$, found 380.1268 . 
(1-(4-Cyanophenyl)-1,2,3,4-tetrahydronaphthalen-2-yl)(phenyl)methanone (4ae).

To a stirred solution of $\mathbf{3 a}(112 \mathrm{mg}, 0.5 \mathrm{mmol})$ and 4-cyanobenzaldehyde $(131 \mathrm{mg}, 1.0 \mathrm{mmol})$ in a mixed solvent of hexane and dichloromethane $(9$ : $1(\mathrm{v} / \mathrm{v}), 2 \mathrm{~mL})$ was added $\mathrm{BF}_{3} \cdot \mathrm{OEt}_{2}(180 \mu \mathrm{L}, 1.4 \mathrm{mmol})$. The mixture was refluxed with an oil bath for $1 \mathrm{~h}$. The reaction mixture was then quenched by $\mathrm{Na}_{2} \mathrm{CO}_{3}$ aqueous solution, and extracted by dichloromethane. The extracts were dried with $\mathrm{Na}_{2} \mathrm{SO}_{4}$, and concentrated in vacuo. The crude<smiles>N#Cc1ccc(C2c3ccccc3CC[C@H]2C(=O)c2ccccc2)cc1</smiles>
mixture was purified by column chromatography $\left(\mathrm{SiO}_{2}\right.$, eluent: $10 \%$ ethyl acetate in hexane) to give 4 ae $(164.5 \mathrm{mg}, 0.49 \mathrm{mmol}$, 97\% yield); Rf: 0.26 (10\% ethyl acetate in hexane); ${ }^{1} \mathrm{H}$ NMR (600 MHz, $\left.\mathrm{CDCl}_{3}\right) \delta 7.74(\mathrm{~d}, J=7.6 \mathrm{~Hz}, 2 \mathrm{H}), 7.52-7.47(\mathrm{~m}, 3 \mathrm{H}), 7.38(\mathrm{t}, J=7.9$ $\mathrm{Hz}, 2 \mathrm{H}), 7.29-7.26(\mathrm{~m}, 2 \mathrm{H}), 7.14(\mathrm{~m}, 2 \mathrm{H}), 7.04(\mathrm{~m}, 1 \mathrm{H}), 6.69(\mathrm{~d}, J=8.3 \mathrm{~Hz}, 1 \mathrm{H}), 4.78(\mathrm{~d}, J=9.6$ $\mathrm{Hz}, 1 \mathrm{H}), 3.85(\mathrm{td}, J=10.3,3.0 \mathrm{~Hz}, 1 \mathrm{H}), 3.10(\mathrm{~m}, 1 \mathrm{H}), 2.93(\mathrm{dt}, J=17.2,4.1 \mathrm{~Hz}, 1 \mathrm{H}), 2.21-2.17(\mathrm{~m}$, $1 \mathrm{H}), 1.94(\mathrm{~m}, 1 \mathrm{H}) ;{ }^{13} \mathrm{C}\left\{{ }^{1} \mathrm{H}\right\} \mathrm{NMR}\left(150 \mathrm{MHz}, \mathrm{CDCl}_{3}\right) \delta 201.5,151.2,137.8,136.1,136.0,133.3$, 132.2, 130.2, 129.9, 128.8, 128.7, 128.0, 126.4, 126.3, 118.8, 110.3, 50.8, 47.1, 29.3, 27.1; HRMS (ESI-TOF) $m / z$ calcd for $\mathrm{C}_{24} \mathrm{H}_{19} \mathrm{NNaO}(\mathrm{M}+\mathrm{Na})^{+} 360.1364$, found 360.1363 .

\section{(1,2,3,4-Tetrahydro-1-(4-methoxyphenyl)naphthalen-2-yl)(phenyl)meth} anone (4af).

To a stirred solution of 3a (112 mg, $0.5 \mathrm{mmol})$ and 4-methoxybenzaldehyde $(75 \mathrm{mg}, 0.55 \mathrm{mmol})$ in a mixed solvent of hexane and dichloromethane $(9$ : $1(\mathrm{v} / \mathrm{v}), 2 \mathrm{~mL})$ was added $\mathrm{BF}_{3} \cdot \mathrm{OEt}_{2}(50 \mu \mathrm{L}, 0.4 \mathrm{mmol})$. The mixture was refluxed with an oil bath for $2 \mathrm{~h}$. The reaction mixture was then quenched<smiles>COc1ccc(C2c3ccccc3CC[C@H]2C(=O)c2ccccc2)cc1</smiles>
by $\mathrm{Na}_{2} \mathrm{CO}_{3}$ aqueous solution, and extracted by dichloromethane. The extracts were dried with $\mathrm{Na}_{2} \mathrm{SO}_{4}$, and concentrated in vacuo. The crude mixture was purified by column chromatography ( $\mathrm{SiO}_{2}$, eluent: $10 \%$ ethyl acetate in hexane) to give 4 af $(82.9 \mathrm{mg}, 0.24 \mathrm{mmol}, 48 \%$ yield); Rf: 0.39 (10\% ethyl acetate in hexane); ${ }^{1} \mathrm{H}$ NMR $\left(600 \mathrm{MHz}, \mathrm{CDCl}_{3}\right) \delta 7.74(\mathrm{~d}, J=7.6 \mathrm{~Hz}, 2 \mathrm{H}), 7.47(\mathrm{t}, J=$ $7.6 \mathrm{~Hz}, 1 \mathrm{H}), 7.35(\mathrm{t}, J=6.9 \mathrm{~Hz}, 2 \mathrm{H}), 7.13-7.09(\mathrm{~m}, 2 \mathrm{H}), 7.06-7.02(\mathrm{~m}, 3 \mathrm{H}), 6.83(\mathrm{~d}, J=7.6 \mathrm{~Hz}, 1 \mathrm{H})$, $6.72(\mathrm{~d}, J=8.3 \mathrm{~Hz}, 2 \mathrm{H}), 4.61(\mathrm{~d}, J=9.6 \mathrm{~Hz}, 1 \mathrm{H}), 3.88(\mathrm{~m}, 1 \mathrm{H}), 3.71(\mathrm{~s}, 3 \mathrm{H}), 3.06(\mathrm{~m}, 1 \mathrm{H}), 2.90(\mathrm{~m}$, $1 \mathrm{H}), 2.14(\mathrm{~m}, 1 \mathrm{H}), 1.96(\mathrm{~m}, 1 \mathrm{H}) ;{ }^{13} \mathrm{C}\left\{{ }^{1} \mathrm{H}\right\} \mathrm{NMR}\left(150 \mathrm{MHz}, \mathrm{CDCl}_{3}\right) \delta 202.7,158.0,139.6,137.5$, 136.7, 135.9, 132.8, 130.2, 130.1, 128.5, 128.4, 128.1, 126.0, 125.8, 113.7, 55.1, 51.0, 46.4, 29.3, 27.1; HRMS (ESI-TOF) $m / z$ calcd for $\mathrm{C}_{24} \mathrm{H}_{22} \mathrm{NaO}_{2}(\mathrm{M}+\mathrm{Na})^{+} 365.1517$, found 365.1516 . 
(1-(4-(Dimethylamino)phenyl)-1,2,3,4-tetrahydronaphthalen-2-yl)(phenyl)methanone (4ag).

To a stirred solution of $\mathbf{3 a} \quad\left(\begin{array}{lllll}56 & \mathrm{mg}, & 0.25 & \mathrm{mmol}\end{array}\right)$ and 4-dimethylaminobenzaldehyde ( $45 \mathrm{mg}, 0.3 \mathrm{mmol})$ in dichloromethane $(1 \mathrm{~mL})$ was added TMSOTf ( $90 \mu \mathrm{L}, 0.5 \mathrm{mmol})$. The mixture was refluxed with an oil bath for $4 \mathrm{~h}$. The reaction mixture was then quenched by $\mathrm{Na}_{2} \mathrm{CO}_{3}$ aqueous solution, and extracted by dichloromethane. The extracts were dried with $\mathrm{Na}_{2} \mathrm{SO}_{4}$, and concentrated in vacuo. The crude mixture was purified by column chromatography ( $\mathrm{SiO}_{2}$, eluent: $20 \%$ ethyl acetate in hexane) to give $\mathbf{4 a g}$ (26.7<smiles>CN(C)c1ccc(C2c3ccccc3CC[C@H]2C(=O)c2ccccc2)cc1</smiles>
$\mathrm{mg}, 0.075 \mathrm{mmol}, 30 \%$ yield); Rf: 0.35 (20\% ethyl acetate in hexane); ${ }^{1} \mathrm{H}$ NMR (600 MHz, $\left.\mathrm{CDCl}_{3}\right) \delta$ $7.76(\mathrm{~d}, J=7.6 \mathrm{~Hz}, 2 \mathrm{H}), 7.46$ (t, $J=7.6 \mathrm{~Hz}, 1 \mathrm{H}), 7.35$ (t, $J=7.6 \mathrm{~Hz}, 2 \mathrm{H}), 7.11-7.08$ (m, 2H), 7.02 (t, $J=7.6 \mathrm{~Hz}, 1 \mathrm{H}), 6.99(\mathrm{~d}, J=9.0 \mathrm{~Hz}, 2 \mathrm{H}), 6.88(\mathrm{~d}, J=7.6 \mathrm{~Hz}, 1 \mathrm{H}), 6.58(\mathrm{~d}, J=9.0 \mathrm{~Hz}, 2 \mathrm{H}), 4.57(\mathrm{~d}$, $J=8.3 \mathrm{~Hz}, 1 \mathrm{H}), 3.89(\mathrm{td}, J=10.3,3.4 \mathrm{~Hz}, 1 \mathrm{H}), 3.04(\mathrm{ddd}, J=16.5,11.0,5.5 \mathrm{~Hz}, 1 \mathrm{H}), 2.88(\mathrm{dt}, J=$ 16.5, $4.1 \mathrm{~Hz}, 1 \mathrm{H}), 2.84(\mathrm{~s}, 6 \mathrm{H}), 2.14(\mathrm{~m}, 1 \mathrm{H}), 1.95(\mathrm{~m}, 1 \mathrm{H}) ;{ }^{13} \mathrm{C}\left\{{ }^{1} \mathrm{H}\right\} \mathrm{NMR}\left(150 \mathrm{MHz}, \mathrm{CDCl}_{3}\right) \delta$ 202.9, 148.9, 139.9, 136.8, 135.9, 132.7, 130.1, 129.8, 128.4, 128.3, 128.2, 125.9, 125.6, 112.7, 50.9, 46.1, 40.7, 29.2, 26.9; HRMS (ESI-TOF) $\mathrm{m} / z$ calcd for $\mathrm{C}_{25} \mathrm{H}_{25} \mathrm{NNaO}(\mathrm{M}+\mathrm{Na})^{+} 378.1834$, found 378.1828 .

\section{(1,2,3,4-Tetrahydro-1-(4-hydroxyphenyl)naphthalen-2-yl)(phenyl)methanone (4ah).}

To a stirred solution of 3a (56 mg, $0.25 \mathrm{mmol})$ and 4-hydroxybenzaldehyde (37 mg, $0.3 \mathrm{mmol})$ in a mixed solvent of hexane and dichloromethane $(9: 1$ $(\mathrm{v} / \mathrm{v}), 1 \mathrm{~mL})$ was added $\mathrm{BF}_{3} \cdot \mathrm{OEt}_{2}(50 \mu \mathrm{L}, 0.4 \mathrm{mmol})$. The mixture was refluxed with an oil bath for $4 \mathrm{~h}$. The reaction mixture was then quenched by $\mathrm{Na}_{2} \mathrm{CO}_{3}$ aqueous solution, and extracted by dichloromethane. The extracts were dried with $\mathrm{Na}_{2} \mathrm{SO}_{4}$, and concentrated in vacuo. The crude mixture was<smiles>O=C(c1ccccc1)[C@H]1CCc2ccccc2C1c1ccc(O)cc1</smiles>
purified by column chromatography $\left(\mathrm{SiO}_{2}\right.$, eluent: $10-20 \%$ ethyl acetate in hexane) to give $4 \mathbf{a h}\left(61.0 \mathrm{mg}, 0.19 \mathrm{mmol}, 74 \%\right.$ yield); Rf: 0.26 (20\% ethyl acetate in hexane); ${ }^{1} \mathrm{H}$ NMR (600 MHz, $\left.\mathrm{CDCl}_{3}\right) \delta 7.74(\mathrm{~d}, J=7.6 \mathrm{~Hz}, 2 \mathrm{H}), 7.47(\mathrm{t}, J=7.6 \mathrm{~Hz}, 1 \mathrm{H}), 7.35(\mathrm{t}, J=7.6 \mathrm{~Hz}, 2 \mathrm{H})$, 7.13-7.09 (m, 2H), 7.03 (t, $J=7.6 \mathrm{~Hz}, 1 \mathrm{H}), 7.00(\mathrm{~d}, J=9.0 \mathrm{~Hz}, 2 \mathrm{H}), 6.82(\mathrm{~d}, J=7.6 \mathrm{~Hz}, 1 \mathrm{H}), 6.64(\mathrm{~d}$, $J=9.0 \mathrm{~Hz}, 2 \mathrm{H}), 4.60(\mathrm{~d}, J=9.6 \mathrm{~Hz}, 1 \mathrm{H}), 3.87(\mathrm{~m}, 1 \mathrm{H}), 3.06(\mathrm{~m}, 1 \mathrm{H}), 2.90(\mathrm{dt}, J=17.2,4.8 \mathrm{~Hz}, 1 \mathrm{H})$, $2.13(\mathrm{~m}, 1 \mathrm{H}), 1.95(\mathrm{~m}, 1 \mathrm{H}) \quad{ }^{13} \mathrm{C}\left\{{ }^{1} \mathrm{H}\right\} \mathrm{NMR}\left(150 \mathrm{MHz}, \mathrm{CDCl}_{3}\right) \delta 202.8,153.9,139.5,137.6,136.7$, 135.9, 132.9, 130.4, 130.0, 128.6, 128.5, 128.1, 126.0, 125.8, 115.2, 51.0, 46.4, 29.3, 27.1; HRMS (ESI-TOF) $m / z$ calcd for $\mathrm{C}_{23} \mathrm{H}_{20} \mathrm{NaO}_{2}(\mathrm{M}+\mathrm{Na})^{+} 351.1361$, found 351.1354 . 
(1-(3-Bromophenyl)-1,2,3,4-tetrahydronaphthalen-2-yl)(phenyl)methanone (4ai).

To a stirred solution of 3a $(56 \mathrm{mg}, 0.25 \mathrm{mmol})$ and 3-bromobenzaldehyde (56 $\mathrm{mg}, 0.3 \mathrm{mmol})$ in a mixed solvent of hexane and dichloromethane $(9: 1$ $(\mathrm{v} / \mathrm{v}), 1 \mathrm{~mL})$ was added $\mathrm{BF}_{3} \cdot \mathrm{OEt}_{2}(25 \mu \mathrm{L}, 0.2 \mathrm{mmol})$. The mixture was refluxed with an oil bath for $2 \mathrm{~h}$. The reaction mixture was then quenched by $\mathrm{Na}_{2} \mathrm{CO}_{3}$ aqueous solution, and extracted by dichloromethane. The extracts<smiles>O=C(c1ccccc1)[C@H]1CCc2ccccc2C1c1cccc(Br)c1</smiles>
were dried with $\mathrm{Na}_{2} \mathrm{SO}_{4}$, and concentrated in vacuo. The crude mixture was purified by column chromatography ( $\mathrm{SiO}_{2}$, eluent: $5 \%$ ethyl acetate in hexane) to give 4ai $(75.2 \mathrm{mg}$, 0.19 mmol, $77 \%$ yield); Rf: 0.52 (10\% ethyl acetate in hexane); ${ }^{1} \mathrm{H}$ NMR (600 MHz, $\left.\mathrm{CDCl}_{3}\right) \delta 7.76$ $(\mathrm{d}, J=7.6 \mathrm{~Hz}, 2 \mathrm{H}), 7.49(\mathrm{t}, J=7.6 \mathrm{~Hz}, 1 \mathrm{H}), 7.38(\mathrm{t}, J=7.6 \mathrm{~Hz}, 2 \mathrm{H}), 7.27(\mathrm{~s}, 1 \mathrm{H}), 7.23(\mathrm{~d}, J=7.6$ Hz, 1H), 7.14-7.11 (m, 2H), 7.09-7.03 (m, 3H), $6.78(\mathrm{~d}, J=7.6 \mathrm{~Hz}, 1 \mathrm{H}), 4.65$ (d, $J=9.6 \mathrm{~Hz}, 1 \mathrm{H})$, $3.86(\mathrm{~m}, 1 \mathrm{H}), 3.06(\mathrm{~m}, 1 \mathrm{H}), 2.90(\mathrm{dt}, J=16.5,4.1 \mathrm{~Hz}, 1 \mathrm{H}), 2.16(\mathrm{~m}, 1 \mathrm{H}), 1.93(\mathrm{~m}, 1 \mathrm{H})$; ${ }^{13} \mathrm{C}\left\{{ }^{1} \mathrm{H}\right\}$ NMR $\left(150 \mathrm{MHz}, \mathrm{CDCl}_{3}\right) \delta 201.9,147.9,138.3,136.4,135.9,133.0,132.1,130.0,129.9$, 129.5, 128.6, 128.6, 128.2, 128.1, 126.2, 126.1, 122.5, 50.7, 46.8, 29.1, 27.0; HRMS (ESI-TOF) $\mathrm{m} / \mathrm{z}$ calcd for $\mathrm{C}_{23} \mathrm{H}_{19} \mathrm{BrNaO}(\mathrm{M}+\mathrm{Na})^{+} 413.0517$, found 413.0514.

\section{(1,2,3,4-Tetrahydro-1-(3-nitrophenyl)naphthalen-2-yl)(phenyl)methanone (4aj).}

To a stirred solution of $\mathbf{3 a}(112 \mathrm{mg}, 0.5 \mathrm{mmol})$ and 3-nitroaldehyde $(83.1 \mathrm{mg}$, $0.55 \mathrm{mmol})$ in a mixed solvent of hexane and dichloromethane $(4: 1(\mathrm{v} / \mathrm{v}), 2$ $\mathrm{mL}$ ) was added $\mathrm{BF}_{3} \cdot \mathrm{OEt}_{2}(50 \mu \mathrm{L}, 0.4 \mathrm{mmol})$. The mixture was stirred refluxed with an oil bath for $2 \mathrm{~h}$. The reaction mixture was then quenched by $\mathrm{Na}_{2} \mathrm{CO}_{3}$ aqueous solution, and extracted by dichloromethane. The extracts<smiles>O=C(c1ccccc1)[C@H]1CCc2ccccc2C1c1cccc([N+](=O)[O-])c1</smiles>
were dried with $\mathrm{Na}_{2} \mathrm{SO}_{4}$, and concentrated in vacuo. The crude mixture was purified by column chromatography $\left(\mathrm{SiO}_{2}\right.$, eluent: $5 \%$ ethyl acetate in hexane) to give 4aj (171.6 $\mathrm{mg}$, $0.46 \mathrm{mmol}, 96 \%$ yield); Rf: 0.31 (10\% ethyl acetate in hexane); ${ }^{1} \mathrm{H} \mathrm{NMR}\left(600 \mathrm{MHz}, \mathrm{CDCl}_{3}\right) \delta 8.00$ $7.97(\mathrm{~m}, 2 \mathrm{H}), 7.77$ (d, $J=7.6 \mathrm{~Hz}, 2 \mathrm{H}), 7.54$ (d, $J=7.6 \mathrm{~Hz}, 1 \mathrm{H}), 7.49$ (t, $J=7.6 \mathrm{~Hz}, 1 \mathrm{H}), 7.39-7.35$ (m, 3H), 7.18-7.13 (m, 2H), 7.04 (t, $J=7.6 \mathrm{~Hz}, 1 \mathrm{H}), 6.69(\mathrm{~d}, J=7.6 \mathrm{~Hz}, 1 \mathrm{H}), 4.84(\mathrm{~d}, J=10.3 \mathrm{~Hz}$, $1 \mathrm{H}), 3.90(\mathrm{t}, J=10.3 \mathrm{~Hz}, 1 \mathrm{H}), 3.14(\mathrm{~m}, 1 \mathrm{H}), 2.95(\mathrm{~m}, 1 \mathrm{H}), 2.22(\mathrm{~m}, 1 \mathrm{H}), 1.95(\mathrm{~m}, 1 \mathrm{H})$; ${ }^{13} \mathrm{C}\left\{{ }^{1} \mathrm{H}\right\}$ NMR $\left(150 \mathrm{MHz}, \mathrm{CDCl}_{3}\right) \delta 201.4,148.4,147.6,137.7,136.04,136.02,133.3,129.8,129.2$, 128.9, 128.7, 128.1, 126.5, 126.4, 123.9, 121.6, 50.8, 46.7, 29.3, 27.2; HRMS (ESI-TOF) $\mathrm{m} / \mathrm{z}$ calcd for $\mathrm{C}_{23} \mathrm{H}_{19} \mathrm{NNaO}_{3}(\mathrm{M}+\mathrm{Na})^{+} 380.1263$, found 380.1269 . 


\section{(1,2,3,4-Tetrahydro-1-(3-methoxyphenyl)naphthalen-2-yl)(phenyl)methanone (4ak).}

To a stirred solution of $\mathbf{3 a} \quad(112 \mathrm{mg}, \quad 0.5 \quad \mathrm{mmol})$ and 3-methoxybenzaldehyde ( $75 \mathrm{mg}, 0.55 \mathrm{mmol}$ ) in a mixed solvent of hexane and dichloromethane $(9: 1(\mathrm{v} / \mathrm{v}), 2 \mathrm{~mL})$ was added $\mathrm{BF}_{3} \cdot \mathrm{OEt}_{2}(50 \mu \mathrm{L}, 0.4$ $\mathrm{mmol})$. The mixture was refluxed with an oil bath for $2 \mathrm{~h}$. The reaction mixture was then quenched by $\mathrm{Na}_{2} \mathrm{CO}_{3}$ aqueous solution, and extracted by dichloromethane. The extracts were dried with $\mathrm{Na}_{2} \mathrm{SO}_{4}$, and concentrated<smiles>COc1cccc(C2c3ccccc3CC[C@H]2C(=O)c2ccccc2)c1</smiles>
in vacuo. The crude mixture was purified by column chromatography $\left(\mathrm{SiO}_{2}\right.$, eluent: $10 \%$ ethyl acetate in hexane) to give $\mathbf{4 a k}(103.1 \mathrm{mg}, 0.29 \mathrm{mmol} .58 \%$ yield); Rf: 0.38 (10\% ethyl acetate in hexane); ${ }^{1} \mathrm{H} \mathrm{NMR}\left(600 \mathrm{MHz}, \mathrm{CDCl}_{3}\right) \delta 7.75(\mathrm{~d}, J=7.6 \mathrm{~Hz}, 2 \mathrm{H}), 7.47$ (t, $\left.J=7.6 \mathrm{~Hz}, 1 \mathrm{H}\right), 7.35$ (t, $J=$ $7.6 \mathrm{~Hz}, 2 \mathrm{H}), 7.13-7.08(\mathrm{~m}, 3 \mathrm{H}), 7.03$ (t, $J=7.6 \mathrm{~Hz}, 1 \mathrm{H}), 6.84(\mathrm{~d}, J=8.3 \mathrm{~Hz}, 1 \mathrm{H}), 6.72$ (d, $J=7.6$ $\mathrm{Hz}, 1 \mathrm{H}), 6.67$ (t, $J=2.1 \mathrm{~Hz}, 1 \mathrm{H}), 6.64(\mathrm{dd}, J=8.3,2.1 \mathrm{~Hz}, 1 \mathrm{H}), 4.64(\mathrm{~d}, J=9.0 \mathrm{~Hz}, 1 \mathrm{H}), 3.91(\mathrm{td}, J$ $=10.3,2.8 \mathrm{~Hz}, 1 \mathrm{H}), 3.69$ (s, 3H), 3.05 (ddd, $J=16.8,11.0,4.8 \mathrm{~Hz}, 1 \mathrm{H}), 2.89$ (dt, $J=16.8,4.5 \mathrm{~Hz}$, 1H), $2.15(\mathrm{~m}, 1 \mathrm{H}), 1.95(\mathrm{~m}, 1 \mathrm{H}) ;{ }^{13} \mathrm{C}\left\{{ }^{1} \mathrm{H}\right\} \mathrm{NMR}\left(150 \mathrm{MHz}, \mathrm{CDCl}_{3}\right) \delta 202.5,159.8,147.2,139.1$, 137.1, 136.0, 132.7, 130.2, 129.3, 128.5, 128.1, 126.1, 125.9, 121.8, 115.4, 111.8, 55.2, 50.9, 47.4, 29.2, 27.0; HRMS (ESI-TOF) $\mathrm{m} / z$ calcd for $\mathrm{C}_{24} \mathrm{H}_{22} \mathrm{NaO}_{2}(\mathrm{M}+\mathrm{Na})^{+} 365.1517$, found 365.1514 .

\section{(1-(2-Bromophenyl)-1,2,3,4-tetrahydronaphthalen-2-yl)(phenyl)methanone (4al).}

To a stirred solution of $\mathbf{3 a}(56 \mathrm{mg}, 0.25 \mathrm{mmol})$ and 2-bromobenzaldehyde (56 $\mathrm{mg}, 0.3 \mathrm{mmol})$ in a mixed solvent of hexane and dichloromethane $(9: 1(\mathrm{v} / \mathrm{v})$, $1 \mathrm{~mL}$ ) was added $\mathrm{BF}_{3} \cdot \mathrm{OEt}_{2}(25 \mu \mathrm{L}, 0.2 \mathrm{mmol})$. The mixture was refluxed with an oil bath for $2 \mathrm{~h}$. The reaction mixture was then quenched by $\mathrm{Na}_{2} \mathrm{CO}_{3}$ aqueous solution, and extracted by dichloromethane. The extracts were dried with $\mathrm{Na}_{2} \mathrm{SO}_{4}$, and concentrated in vacuo. The crude mixture was purified by<smiles>O=C(c1ccccc1)[C@H]1CCc2ccccc2C1c1ccccc1Br</smiles>
column chromatography ( $\mathrm{SiO}_{2}$, eluent: $5 \%$ ethyl acetate in hexane) to give 4al ( $85.1 \mathrm{mg}, 0.22 \mathrm{mmol}$, $87 \%$ yield) as a white solid; Rf: $0.6\left(10 \%\right.$ ethyl acetate in hexane); $\mathrm{mp}=129.1-131.7{ }^{\circ} \mathrm{C} ;{ }^{1} \mathrm{H}$ NMR $\left(600 \mathrm{MHz}, \mathrm{CDCl}_{3}\right) \delta 7.82(\mathrm{~d}, J=7.6 \mathrm{~Hz}, 2 \mathrm{H}), 7.50-7.46(\mathrm{~m}, 2 \mathrm{H}), 7.37$ (t, $\left.J=7.6 \mathrm{~Hz}, 2 \mathrm{H}\right), 7.14-7.11$ (m, 3H), 7.06-6.98 (m, 3H), $6.80(\mathrm{~d}, J=7.6 \mathrm{~Hz}, 1 \mathrm{H}), 5.06(\mathrm{~d}, J=6.9 \mathrm{~Hz}, 1 \mathrm{H}), 4.15(\mathrm{~m}, 1 \mathrm{H}), 3.00(\mathrm{~m}$, $1 \mathrm{H}), 2.81(\mathrm{dt}, J=17.2,5.5 \mathrm{~Hz}, 1 \mathrm{H}), 2.15(\mathrm{~m}, 1 \mathrm{H}), 2.03(\mathrm{~m}, 1 \mathrm{H}) ;{ }^{13} \mathrm{C}\left\{{ }^{1} \mathrm{H}\right\}$ NMR $\left(150 \mathrm{MHz}, \mathrm{CDCl}_{3}\right.$, at $\left.50{ }^{\circ} \mathrm{C}\right) \delta 201.9,144.5,137.9,136.7,136.1,133.4,132.8,132.0,129.5,128.5,128.3,128.2,128.0$, $127.4,126.3,126.0,124.7,47.9,46.3,28.2,25.6$; HRMS (ESI-TOF) $m / z$ calcd for $\mathrm{C}_{23} \mathrm{H}_{19} \mathrm{BrNaO}(\mathrm{M}$ $+\mathrm{Na})^{+} 413.0517$, found 413.0526 . 
Table S2. Crystal data and structure refinement for $\mathbf{4 a l}$.

\begin{tabular}{llll}
\hline Formula & $\mathrm{C}_{23} \mathrm{H}_{19} \mathrm{OBr}$ & $V\left(\AA^{3}\right)$ & $1808.49(7)$ \\
Fw & 391.29 & $Z$ & 4 \\
Crystal system & monoclinic & $D_{\text {calcd }}\left(\mathrm{g} / \mathrm{cm}^{-3}\right)$ & 1.437 \\
Space group & $P 2_{1} / \mathrm{n}$ & $\mu\left(\mathrm{mm}^{-1}\right)$ & 2.28 \\
$a(\AA)$ & $9.8918(2)$ & $\theta_{\max }\left({ }^{\circ}\right)$ & 30.51 \\
$b(\AA)$ & $10.5226(2)$ & no. of data collected & 5385 \\
$c(\AA)$ & $17.7799(4)$ & no. of parameters & 226 \\
$\alpha\left({ }^{\circ}\right)$ & 90.000 & $R$ & 0.0584 \\
$\beta\left({ }^{\circ}\right)$ & $102.256(2)$ & $R \mathrm{w}$ & 0.1857 \\
$\gamma\left({ }^{\circ}\right)$ & 90.000 & & \\
\hline
\end{tabular}

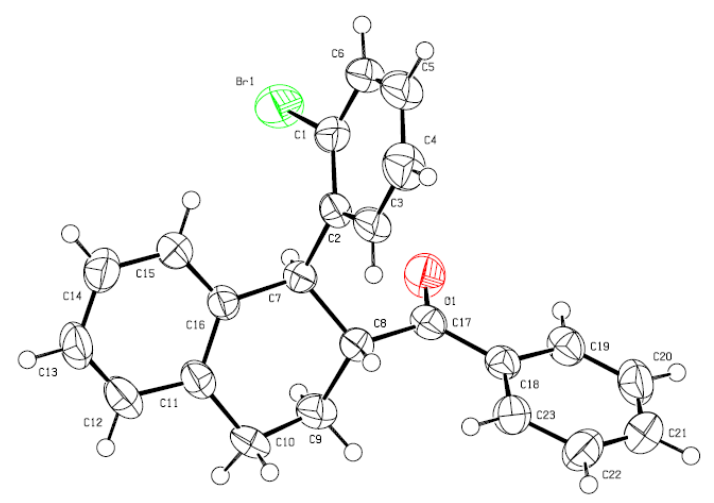

Figure S1. An ORTEP view (50\% probability level) of 4al

\section{(1,2,3,4-Tetrahydro-1-(2-methoxyphenyl)naphthalen-2-yl)(phenyl)methanone (4am).}

To stirred solution of $\mathbf{3 a}(112 \mathrm{mg}, 0.5 \mathrm{mmol})$ and 2-methoxybenzaldehyde (75 mg, $0.55 \mathrm{mmol}$ ) in a mixed solvent of hexane and dichloromethane (9 : $1(\mathrm{v} / \mathrm{v}), 2 \mathrm{~mL})$ was added $\mathrm{BF}_{3} \cdot \mathrm{OEt}_{2}(50 \mu \mathrm{L}, 0.4 \mathrm{mmol})$. The mixture was refluxed with an oil bath for $2 \mathrm{~h}$. The reaction mixture was then quenched by $\mathrm{Na}_{2} \mathrm{CO}_{3}$ aqueous solution, and extracted by dichloromethane.<smiles>COc1ccccc1C1c2ccccc2CC[C@H]1C(=O)c1ccccc1</smiles>
The extracts were dried with $\mathrm{Na}_{2} \mathrm{SO}_{4}$, and concentrated in vacuo. The crude mixture was purified by column chromatography ( $\mathrm{SiO}_{2}$, eluent: $30 \%$ ethyl acetate in hexane) to give 4am (119.9 mg, $0.35 \mathrm{mmol} .70 \%$ yield) as a colorless oil; Rf: 0.39 (10\% ethyl acetate in hexane); ${ }^{1} \mathrm{H}$ NMR (600 MHz, $\left.\mathrm{CDCl}_{3}\right) \delta 7.79(\mathrm{~d}, J=7.6 \mathrm{~Hz}, 2 \mathrm{H}), 7.47(\mathrm{t}, J=7.6 \mathrm{~Hz}, 1 \mathrm{H}), 7.35(\mathrm{t}, J=$ $7.6 \mathrm{~Hz}, 2 \mathrm{H}), 7.13-7.07(\mathrm{~m}, 3 \mathrm{H}), 7.01(\mathrm{t}, J=7.2 \mathrm{~Hz}, 1 \mathrm{H}), 6.89$ (d, $J=7.6 \mathrm{~Hz}, 1 \mathrm{H}), 6.82(\mathrm{~d}, J=8.3$ 
Hz, 1H), 6.79-6.76 (m, 2H), 4.89 (d, $J=7.6 \mathrm{~Hz}, 1 \mathrm{H}), 4.07$ (m, 1H), $3.63(\mathrm{~s}, 3 \mathrm{H}), 2.96$ (ddd, $J=16.5$, 9.0, $4.8 \mathrm{~Hz}, 1 \mathrm{H}), 2.83(\mathrm{dt}, J=16.5,5.5 \mathrm{~Hz}, 1 \mathrm{H}), 2.11(\mathrm{~m}, 1 \mathrm{H}), 1.98(\mathrm{~m}, 1 \mathrm{H}) ;{ }^{13} \mathrm{C}\left\{{ }^{1} \mathrm{H}\right\} \mathrm{NMR}(150$ $\mathrm{MHz}, \mathrm{CDCl}_{3}$ at $\left.50{ }^{\circ} \mathrm{C}\right) \delta 202.9,157.4,139.2,137.2,136.3,134.1,132.5,131.0,129.3,128.4,128.3$, 128.2, 127.6, 126.0, 125.5, 120.7, 111.5, 55.5, 47.9, 42.2, 28.7, 26.2; HRMS (ESI-TOF) $\mathrm{m} / \mathrm{z}$ calcd for $\mathrm{C}_{24} \mathrm{H}_{22} \mathrm{NaO}_{2}(\mathrm{M}+\mathrm{Na})^{+}$365.1517, found 365.1522 .

\section{(1,2,3,4-Tetrahydro-1-(naphthalen-1-yl)naphthalen-2-yl)(phenyl)methanone (4an).}

To a stirred solution of 3a $(56 \mathrm{mg}, 0.25 \mathrm{mmol})$ and 1-naphthaldehyde (47 mg, $0.3 \mathrm{mmol})$ in a mixed solvent of hexane and dichloromethane $(9: 1(\mathrm{v} / \mathrm{v}), 1$ $\mathrm{mL})$ was added $\mathrm{BF}_{3} \cdot \mathrm{OEt}_{2}(25 \mu \mathrm{L}, 0.2 \mathrm{mmol})$. The mixture was refluxed with an oil bath for $2 \mathrm{~h}$. The reaction mixture was then quenched by $\mathrm{Na}_{2} \mathrm{CO}_{3}$ aqueous solution, and extracted by dichloromethane. The extracts were dried with $\mathrm{Na}_{2} \mathrm{SO}_{4}$, and concentrated in vacuo. The crude mixture purified by column<smiles>O=C(c1ccccc1)[C@H]1CCc2ccccc2C1c1cccc2ccccc12</smiles>
chromatography ( $\mathrm{SiO}_{2}$, eluent: $5 \%$ ethyl acetate in hexane) to give $4 \mathbf{a n}(62.2 \mathrm{mg}, 0.17 \mathrm{mmol}, 69 \%$ yield); Rf: 0.51 (10\% ethyl acetate in hexane); ${ }^{1} \mathrm{H}$ NMR $\left(600 \mathrm{MHz}, \mathrm{CDCl}_{3}\right.$ at $\left.50{ }^{\circ} \mathrm{C}\right) \delta 7.99(\mathrm{~m}, 1 \mathrm{H})$, $7.79(\mathrm{~m}, 1 \mathrm{H}), 7.64-7.59(\mathrm{~m}, 3 \mathrm{H}), 7.41-7.37(\mathrm{~m}, 3 \mathrm{H}), 7.27(\mathrm{t}, J=7.6 \mathrm{~Hz}, 1 \mathrm{H}), 7.24-7.22(\mathrm{~m}, 2 \mathrm{H})$, $7.18-7.16(\mathrm{~m}, 2 \mathrm{H}), 7.11(\mathrm{t}, J=7.6 \mathrm{~Hz}, 1 \mathrm{H}), 6.97(\mathrm{t}, J=7.6 \mathrm{~Hz}, 1 \mathrm{H}), 6.80(\mathrm{~d}, J=7.6 \mathrm{~Hz}, 1 \mathrm{H}), 5.38$ (d, $J=6.9 \mathrm{~Hz}, 1 \mathrm{H}), 4.23(\mathrm{~m}, 1 \mathrm{H}), 3.10(\mathrm{~m}, 1 \mathrm{H}), 2.92(\mathrm{dt}, J=16.5,4.8 \mathrm{~Hz}, 1 \mathrm{H}), 2.21(\mathrm{~m}, 1 \mathrm{H}), 2.11(\mathrm{~m}$, $1 \mathrm{H}) ;{ }^{13} \mathrm{C}\left\{{ }^{1} \mathrm{H}\right\} \mathrm{NMR}\left(150 \mathrm{MHz}, \mathrm{CDCl}_{3}\right.$ at $\left.50{ }^{\circ} \mathrm{C}\right) \delta 202.7,139.4,136.8,135.7,134.3,132.6,131.7$, 129.8, 129.0, 128.6, 128.4, 128.0, 127.9, 127.3, 126.4, 126.0, 125.9, 125.4, 125.3, 124.2, 49.1, 29.7, 28.6, 26.5; HRMS (ESI-TOF) $\mathrm{m} / z$ calcd for $\mathrm{C}_{27} \mathrm{H}_{22} \mathrm{NaO}(\mathrm{M}+\mathrm{Na})^{+} 385.1568$, found 385.1571 .

\section{(1,2,3,4-Tetrahydro-1-styrylnaphthalen-2-yl)(phenyl)methanone (4ao).}

To a stirred solution of $\mathbf{3 a}(112 \mathrm{mg}, 0.5 \mathrm{mmol})$ and trans-cinnamilaldehyde $(0.19 \mathrm{~mL}, 1.5 \mathrm{mmol})$ in a mixed solvent of hexane and dichloromethane $(9$ : 1 (v/v), $2 \mathrm{~mL})$ was added $\mathrm{BF}_{3} \cdot \mathrm{OEt}_{2}(0.15 \mathrm{~mL}, 1.5 \mathrm{mmol})$. The mixture was stirred refluxed with an oil bath for $6 \mathrm{~h}$. The reaction mixture was then quenched by water, and extracted by dichloromethane. The extracts were<smiles>O=C(c1ccccc1)[C@H]1CCc2ccccc2C1/C=C/c1ccccc1</smiles>
dried with $\mathrm{Na}_{2} \mathrm{SO}_{4}$, and concentrated in vacuo. The crude mixture was purified by column chromatography ( $\mathrm{SiO}_{2}$, eluent: 5-10\% ethyl acetate in hexane) to give $4 \mathbf{a o}$ (89.7 $\mathrm{mg}, 53 \%$ yield) as an oil; Rf: $0.39,10 \%$ ethyl acetate in hexane; ${ }^{1} \mathrm{H}$ NMR (600 MHz, $\left.\mathrm{CDCl}_{3}\right) \delta 7.92$ (d, $J=7.6 \mathrm{~Hz}, 2 \mathrm{H}), 7.51(\mathrm{t}, J=7.6 \mathrm{~Hz}, 1 \mathrm{H}), 7.42(\mathrm{t}, J=7.6 \mathrm{~Hz}, 2 \mathrm{H}), 7.29-7.13(\mathrm{~m}, 9 \mathrm{H}), 6.50$ (d, $J=$ $15.1 \mathrm{~Hz}, 1 \mathrm{H}), 6.07(\mathrm{dd}, J=15.1,9.0 \mathrm{~Hz}, 1 \mathrm{H}), 4.20(\mathrm{t}, J=9.0 \mathrm{~Hz}, 1 \mathrm{H}), 3.74(\mathrm{t}, J=9.0 \mathrm{~Hz}, 1 \mathrm{H}), 2.98$ $(\mathrm{m}, 1 \mathrm{H}), 2.89(\mathrm{~m}, 1 \mathrm{H}), 2.16(\mathrm{~m}, 1 \mathrm{H}), 1.95(\mathrm{~m}, 1 \mathrm{H}) ;{ }^{13} \mathrm{C}\left\{{ }^{1} \mathrm{H}\right\} \mathrm{NMR}\left(150 \mathrm{MHz}, \mathrm{CDCl}_{3}\right) \delta 202.3,137.6$, 137.1, 136.8, 135.5, 133.0, 132.8, 131.8, 129.4, 128.8, 128.7, 128.4, 128.3, 127.2, 126.2, 126.1, 47.8, 44.7, 29.0, 26.5; HRMS (ESI-TOF) $\mathrm{m} / z$ calcd for $\mathrm{C}_{25} \mathrm{H}_{22} \mathrm{NaO}(\mathrm{M}+\mathrm{Na})^{+} 361.1568$, found 361.1574. 


\section{(1-(1-Bromo-2-phenylvinyl)-1,2,3,4-tetrahydronaphthalen-2-yl)(phenyl)methanone (4ap).}

To a stirred solution of $\mathbf{3 a}(112 \mathrm{mg}, 0.5 \mathrm{mmol})$ and $\alpha$-bromocinnamaldehyde $(211 \mathrm{mg}, 1.0 \mathrm{mmol})$ in dichloromethane $(2 \mathrm{~mL})$ was added $\mathrm{Me}_{3} \operatorname{SiOTf}(0.18$ $\mathrm{mL}, 1.0 \mathrm{mmol})$. The mixture was refluxed with an oil bath for $3 \mathrm{~h}$. The reaction mixture was then quenched by water, and extracted by dichloromethane. The extracts were dried with $\mathrm{Na}_{2} \mathrm{SO}_{4}$, and concentrated in<smiles>O=C(c1ccccc1)[C@H]1CCc2ccccc2C1/C(Br)=C/c1ccccc1</smiles>
vacuo. The crude mixture was purified by column chromatography $\left(\mathrm{SiO}_{2}\right.$, eluent: 3\% ethyl acetate in hexane) to give $\mathbf{4 a p}(135 \mathrm{mg}, 65 \%$ yield) as an oil; Rf: 0.4 (10\% ethyl acetate in hexane); ${ }^{1} \mathrm{H} \mathrm{NMR}\left(600 \mathrm{MHz}, \mathrm{CDCl}_{3}\right) \delta 8.01(\mathrm{~d}, J=7.6 \mathrm{~Hz}, 2 \mathrm{H}), 7.57(\mathrm{t}, J=7.6 \mathrm{~Hz}, 1 \mathrm{H})$, 7.48-7.43 (m, 4H), $7.33(\mathrm{~m}, 1 \mathrm{H}), 7.26(\mathrm{t}, J=7.6 \mathrm{~Hz}, 2 \mathrm{H}), 7.24-7.18(\mathrm{~m}, 3 \mathrm{H}), 7.14(\mathrm{~m}, 1 \mathrm{H}), 7.01(\mathrm{~s}$, $1 \mathrm{H}), 4.60(\mathrm{~d}, J=9.6 \mathrm{~Hz}, 1 \mathrm{H}), 4.22(\mathrm{td}, J=9.6,3.4 \mathrm{~Hz}, 1 \mathrm{H}), 3.01(\mathrm{ddd}, J=16.5,12.4,4.8 \mathrm{~Hz}, 1 \mathrm{H})$, $2.87(\mathrm{dt}, J=16.5,3.4 \mathrm{~Hz}, 1 \mathrm{H}), 2.18(\mathrm{~m}, 1 \mathrm{H}), 1.93(\mathrm{qd}, J=12.4,4.8 \mathrm{~Hz}, 1 \mathrm{H}) ;{ }^{13} \mathrm{C}\left\{{ }^{1} \mathrm{H}\right\} \mathrm{NMR}(150$ $\left.\mathrm{MHz}, \mathrm{CDCl}_{3}\right) \delta 202.2,136.7,136.4,135.6,135.5,133.3,132.3,129.2,129.0,128.9,128.7,128.4$, 128.0, 127.8, 126.6, 126.4, 52.7, 46.3, 29.2, 26.5; HRMS (ESI-TOF) $\mathrm{m} / \mathrm{z}$ calcd for $\mathrm{C}_{25} \mathrm{H}_{21} \mathrm{BrNaO}(\mathrm{M}$ $+\mathrm{Na})^{+} 439.0673$, found 439.0668 .

\section{1-(1,2,3,4-Tetrahydro-1-phenylnaphthalen-2-yl)ethanone (4b).}

To a stirred solution of $\mathbf{3 b}(81 \mathrm{mg}, 0.5 \mathrm{mmol})$ and benzaldehyde $(90 \mathrm{mg}, 0.85$ $\mathrm{mmol})$ in hexane $(2 \mathrm{~mL})$ was added $\mathrm{BF}_{3} \cdot \mathrm{OEt}_{2}(50 \mu \mathrm{L}, 0.4 \mathrm{mmol})$. The mixture was refluxed with an oil bath for $4 \mathrm{~h}$. The reaction mixture was then<smiles>CC(=O)[C@H]1CCc2ccccc2C1c1ccccc1</smiles>
quenched by $\mathrm{Na}_{2} \mathrm{CO}_{3}$ aqueous solution, and extracted by dichloromethane. The extracts were dried with $\mathrm{Na}_{2} \mathrm{SO}_{4}$, and concentrated in vacuo. The crude mixture was purified by column chromatography $\left(\mathrm{SiO}_{2}\right.$, eluent: $5 \%$ ethyl acetate in hexane) to give $\mathbf{4 b + 4 b}$ ' $(100.2 \mathrm{mg})$; $\mathrm{Rf} \mathbf{4 b}$ : 0.31 (10\% ethyl acetate in hexane); ${ }^{1} \mathrm{H}$ NMR $\left(600 \mathrm{MHz}, \mathrm{CDCl}_{3}\right) \delta$ 7.27-7.24 (m, 2H), 7.21-7.18 (m, 1H), 7.10-7.07 (m, 4H), 7.01-6.99 (m, 1H), 6.75 (d, $J=7.6 \mathrm{~Hz}, 1 \mathrm{H}), 4.37$ (d, $J=9.6 \mathrm{~Hz}, 1 \mathrm{H}), 3.06-2.97$ (m, 2H), 2.90 (dt, $J$ $=16.5,4.8 \mathrm{~Hz}, 1 \mathrm{H}), 2.09(\mathrm{~m}, 1 \mathrm{H}), 1.93(\mathrm{~s}, 3 \mathrm{H}), 1.93-1.88(\mathrm{~m}, 1 \mathrm{H}) ;{ }^{13} \mathrm{C}\left\{{ }^{1} \mathrm{H}\right\} \mathrm{NMR}(150 \mathrm{MHz}$, $\left.\mathrm{CDCl}_{3}\right) \delta 211.0,145.1,138.7,135.8,129.9,129.2,128.8$ 128.5, 126.5, 126.0, 125.9, 56.4, 47.3, 30.0, 29.0, 25.4; HRMS (ESI-TOF) $m / z$ calcd for $\mathrm{C}_{18} \mathrm{H}_{18} \mathrm{NaO}(\mathrm{M}+\mathrm{Na})^{+} 273.1255$, found 273.1264.

\section{1-(1,2,3,4-Tetrahydro-1-phenylnaphthalen-2-yl)-3-phenylprop-2-en-1-one (4b').}

To a stirred solution of $\mathbf{3 b}(41 \mathrm{mg}, 0.25 \mathrm{mmol})$ and benzaldehyde (80 $\mathrm{mg}, 0.75 \mathrm{mmol})$ in dichloromethane $(1 \mathrm{~mL})$ was added TMSOTf ( $45 \mu \mathrm{L}, 0.25 \mathrm{mmol})$. The mixture was refluxed with an oil bath for $6 \mathrm{~h}$. The reaction mixture was then quenched by $\mathrm{Na}_{2} \mathrm{CO}_{3}$<smiles>O=C(/C=C/c1ccccc1)[C@H]1CCc2ccccc2C1c1ccccc1</smiles> 
aqueous solution, and extracted by dichloromethane. The extracts were dried with $\mathrm{Na}_{2} \mathrm{SO}_{4}$, and concentrated in vacuo. The crude mixture was purified by column chromatography $\left(\mathrm{SiO}_{2}\right.$, eluent: $4 \%$ ethyl acetate in hexane) to give $\mathbf{4 b}$ ' $(63.2 \mathrm{mg}, 0.19 \mathrm{mmol}, 75 \%$ yield); Rf $\mathbf{4} \mathbf{b}$ ': 0.32 (10\% ethyl acetate in hexane); ${ }^{1} \mathrm{H}$ NMR (600 MHz, $\left.\mathrm{CDCl}_{3}\right) \delta$ 7.41-7.37 (m, 3H), 7.35-7.30 (m, 3H), 7.22 (d, $J$ $=7.6 \mathrm{~Hz}, 2 \mathrm{H}), 7.15-7.09(\mathrm{~m}, 5 \mathrm{H}), 7.03-7.00(\mathrm{~m}, 1 \mathrm{H}), 6.79(\mathrm{~d}, J=8.3 \mathrm{~Hz}, 1 \mathrm{H}), 6.56(\mathrm{~d}, J=15.8 \mathrm{~Hz}$, $1 \mathrm{H}), 4.51(\mathrm{~d}, J=9.6 \mathrm{~Hz}, 1 \mathrm{H}), 3.33$ (ddd, $J=11.0,9.6,2.8 \mathrm{~Hz}, 1 \mathrm{H}), 3.05$ (ddd, $J=16.5,11.0,4.1 \mathrm{~Hz}$, $1 \mathrm{H}), 2.93(\mathrm{dt}, J=16.5,4.5 \mathrm{~Hz}, 1 \mathrm{H}), 2.17-2.13(\mathrm{~m}, 1 \mathrm{H}), 2.01-1.92(\mathrm{~m}, 1 \mathrm{H}) ;{ }^{13} \mathrm{C}\left\{{ }^{1} \mathrm{H}\right\} \mathrm{NMR}(150$ $\left.\mathrm{MHz}, \mathrm{CDCl}_{3}\right) \delta 201.7,145.4,142.7,139.1,136.1,134.5,130.4,130.1,129.3,128.8,128.5,128.4$, 128.3, 126.4, 126.1, 125.9, 125.4, 54.4, 47.3, 29.2, 26.1; HRMS (ESI-TOF) $\mathrm{m} / \mathrm{z}$ calcd for $\mathrm{C}_{25} \mathrm{H}_{22} \mathrm{NaO}(\mathrm{M}+\mathrm{Na})^{+} 361.1568$, found 361.1566 .

\section{(1,2,3,4-Tetrahydro-1-phenylnaphthalen-2-yl)(1-methyl-1H-imidazol-2-yl)methanone (4c)}

To a stirred solution of $\mathbf{3 c}$ (114 $\mathrm{mg}, 0.5 \mathrm{mmol})$ and benzaldehyde (160 $\mathrm{mg}, 1.5 \mathrm{mmol})$ in dichloromethane $(2 \mathrm{~mL})$ was added TMSOTf $(270$ $\mu \mathrm{L}, 1.5 \mathrm{mmol})$. The mixture was refluxed with an oil bath for $48 \mathrm{~h}$. The reaction mixture was then quenched by $\mathrm{Na}_{2} \mathrm{CO}_{3}$ aqueous solution, and<smiles>Cn1ccnc1C(=O)[C@H]1CCc2ccccc2C1c1ccccc1</smiles>
extracted by dichloromethane. The extracts were dried with $\mathrm{Na}_{2} \mathrm{SO}_{4}$, and concentrated in vacuo. The crude mixture was purified by column chromatography $\left(\mathrm{SiO}_{2}\right.$, eluent: dichloromethane) to give $\mathbf{4 c}$ (143.5 mg, $0.45 \mathrm{mmol}, 90 \%$ yield). Rf: 0.61 (dichloromethane); ${ }^{1} \mathrm{H} \mathrm{NMR}\left(600 \mathrm{MHz}, \mathrm{CDCl}_{3}\right) \delta 7.21-$ $7.15(\mathrm{~m}, 4 \mathrm{H}), 7.12-7.07$ (m, 3H), $7.04(\mathrm{~s}, 1 \mathrm{H}), 7.00$ (t, $J=7.6 \mathrm{~Hz}, 1 \mathrm{H}), 6.91(\mathrm{~s}, 1 \mathrm{H}), 6.76$ (d, $J=7.6$ $\mathrm{Hz}, 1 \mathrm{H}), 4.59$ (d, $J=10.3 \mathrm{~Hz}, 1 \mathrm{H}), 4.39$ (td, $J=10.3,2.8 \mathrm{~Hz}, 1 \mathrm{H}), 3.87$ (s, 3H), 3.18 (ddd, $J=16.5$, 11.0, $5.5 \mathrm{~Hz}, 1 \mathrm{H}), 2.90(\mathrm{dt}, J=16.5,4.1 \mathrm{~Hz}, 1 \mathrm{H}), 2.30(\mathrm{~m}, 1 \mathrm{H}), 1.94(\mathrm{~m}, 1 \mathrm{H}) ;{ }^{13} \mathrm{C}\left\{{ }^{1} \mathrm{H}\right\} \mathrm{NMR}(150$ $\left.\mathrm{MHz}, \mathrm{CDCl}_{3}\right) \delta 194.3,145.2,142.5,139.3,136.1,129.9,129.5,128.8,128.5,128.2,126.9,126.1$, $125.9,125.7,50.7,46.6,36.2,29.2,27.0$; HRMS (ESI-TOF) $m / z$ calcd for $\mathrm{C}_{21} \mathrm{H}_{20} \mathrm{~N}_{2} \mathrm{NaO}(\mathrm{M}+\mathrm{Na})^{+}$ 339.1473 , found 339.1472 .

\section{(1,2,3,4-Tetrahydro-7-methyl-1-phenylnaphthalen-2-yl)(phenyl)methanone (4d).}

To a stirred solution of $\mathbf{3 d}$ (119 mg, $0.5 \mathrm{mmol})$ and benzaldehyde $(63 \mathrm{mg}$, $0.6 \mathrm{mmol})$ in hexane $(2 \mathrm{~mL})$ was added $\mathrm{BF}_{3} \cdot \mathrm{OEt}_{2}(50 \mu \mathrm{L}, 0.4 \mathrm{mmol})$. The mixture was refluxed with an oil bath for $1.5 \mathrm{~h}$. The reaction mixture was then quenched by $\mathrm{Na}_{2} \mathrm{CO}_{3}$ aqueous solution, and extracted by<smiles>Cc1ccc2c(c1)C(c1ccccc1)[C@H](C(=O)c1ccccc1)CC2</smiles>
dichloromethane. The extracts were dried with $\mathrm{Na}_{2} \mathrm{SO}_{4}$, and concentrated in vacuo. The crude mixture was purified by column chromatography $\left(\mathrm{SiO}_{2}\right.$, eluent: $5 \%$ ethyl acetate in hexane) to give 4d (154.2 mg, $0.47 \mathrm{mmol}, 94 \%$ yield); Rf: 0.49 (10\% ethyl acetate in hexane); ${ }^{1} \mathrm{H}$ NMR (600 MHz, $\left.\mathrm{CDCl}_{3}\right) \delta 7.72(\mathrm{~d}, J=7.6 \mathrm{~Hz}, 2 \mathrm{H}), 7.46(\mathrm{t}, J=7.6 \mathrm{~Hz}, 1 \mathrm{H}), 7.34(\mathrm{t}, J=7.6 \mathrm{~Hz}, 2 \mathrm{H}), 7.21-7.16(\mathrm{~m}$, 2H), 7.14-7.08 (m, 3H), $7.02(\mathrm{~d}, J=7.6 \mathrm{~Hz}, 1 \mathrm{H}), 6.93(\mathrm{~d}, J=7.6 \mathrm{~Hz}, 1 \mathrm{H}), 6.63(\mathrm{~s}, 1 \mathrm{H}), 4.62(\mathrm{~d}, J=$ 
$8.9 \mathrm{~Hz}, 1 \mathrm{H}), 3.88(\mathrm{td}, J=10.3,3.4 \mathrm{~Hz}, 1 \mathrm{H}), 3.00(\mathrm{ddd}, J=16.5,10.3,4.8 \mathrm{~Hz}, 1 \mathrm{H}), 2.84(\mathrm{dt}, J=16.5$, $4.8 \mathrm{~Hz}, 1 \mathrm{H}), 2.15(\mathrm{~s}, 3 \mathrm{H}), 2.15-2.11(\mathrm{~m}, 1 \mathrm{H}), 1.97-1.90(\mathrm{~m}, 1 \mathrm{H}) ;{ }^{13} \mathrm{C}\left\{{ }^{1} \mathrm{H}\right\} \mathrm{NMR}\left(150 \mathrm{MHz}, \mathrm{CDCl}_{3}\right)$ $\delta$ 202.7, 145.6, 138.8, 136.7, 135.5, 133.0 132.8, 130.5, 129.3, 128.5, 128.4, 128.1, 126.8, 126.2, 51.1, 47.1, 28.8, 26.9, 21.0; HRMS (ESI-TOF) $m / z$ calcd for $\mathrm{C}_{24} \mathrm{H}_{22} \mathrm{NaO}(\mathrm{M}+\mathrm{Na})^{+} 349.1568$, found 349.1563.

\section{(7-(Trifluoromethyl)-1,2,3,4-tetrahydro-1-phenylnaphthalen-2-yl)(phenyl)methanone (4e).}

To a stirred solution of $\mathbf{3 e}(119 \mathrm{mg}, 0.5 \mathrm{mmol})$ and benzaldehyde $(106 \mathrm{mg}, 1.0 \mathrm{mmol})$ in dichloromethane $(2 \mathrm{~mL})$ was added TMSOTf (90 $\mu \mathrm{L}, 0.5 \mathrm{mmol})$. The mixture was refluxed with an oil bath for 18<smiles>O=C(c1ccccc1)[C@H]1CCc2ccc(C(F)(F)F)cc2C1c1ccccc1</smiles>
h. The reaction mixture was then quenched by $\mathrm{Na}_{2} \mathrm{CO}_{3}$ aqueous solution, and extracted by dichloromethane. The extracts were dried with $\mathrm{Na}_{2} \mathrm{SO}_{4}$, and concentrated in vacuo. The crude mixture was purified by column chromatography $\left(\mathrm{SiO}_{2}\right.$, eluent: $5 \%$ ethyl acetate in hexane) to give $4 \mathbf{e}$ (140 mg, $0.37 \mathrm{mmol}, 74 \%$ yield); Rf: 0.35 (10\% ethyl acetate in hexane); ${ }^{1} \mathrm{H}$ NMR $\left(600 \mathrm{MHz}, \mathrm{CDCl}_{3}\right) \delta 7.73(\mathrm{~d}, J=7.6 \mathrm{~Hz}, 2 \mathrm{H}), 7.48(\mathrm{t}, J=7.6 \mathrm{~Hz}, 1 \mathrm{H}), 7.36(\mathrm{t}, J=7.6 \mathrm{~Hz}, 3 \mathrm{H})$, 7.23-7.19 (m, 3H), 7.13 (t, $J=7.6 \mathrm{~Hz}, 1 \mathrm{H}), 7.10-7.09$ (m, 3H), 4.68 (d, $J=8.3 \mathrm{~Hz}, 1 \mathrm{H}), 3.91$ (ddd, $J=10.3,8.3,3.4 \mathrm{~Hz}, 1 \mathrm{H}), 3.06(\mathrm{ddd}, J=17.2,10.3,4.8 \mathrm{~Hz}, 1 \mathrm{H}), 2.92(\mathrm{dt}, J=17.2,4.8 \mathrm{~Hz}, 1 \mathrm{H})$, $2.16(\mathrm{~m}, 1 \mathrm{H}), 1.98(\mathrm{~m}, 1 \mathrm{H}) ;{ }^{13} \mathrm{C}\left\{{ }^{1} \mathrm{H},{ }^{19} \mathrm{~F}\right\} \mathrm{NMR}\left(150 \mathrm{MHz}, \mathrm{CDCl}_{3}\right) \delta 202.1,144.5,140.0,139.8$, 136.4, 133.0, 129.1, 129.0, 128.6, 128.6, 128.5, 128.1, 127.0, 126.7, 124.2, 122.6, 50.4, 46.9, 28.8, 26.0; HRMS (ESI-TOF) $\mathrm{m} / z$ calcd for $\mathrm{C}_{24} \mathrm{H}_{19} \mathrm{~F}_{3} \mathrm{NaO}(\mathrm{M}+\mathrm{Na})^{+} 403.1286$, found 403.1295 .

\section{Methyl 1,2,3,4-tetrahydro-1-phenylnaphthalene-2-carboxylate (5).}

The dichloromethane solution $(1 \mathrm{~mL})$ containing $4 \mathbf{c}(31 \mathrm{mg}, 0.1 \mathrm{mmol})$ and MS4A (70 mg) was stirred at room temperature under nitrogen for $1.5 \mathrm{~h}$. To the mixture was added MeOTf $(53 \mu \mathrm{L}, 0.5 \mathrm{mmol})$ at $0{ }^{\circ} \mathrm{C}$, and resulting mixture was stirred at room temperature until consumption of<smiles>COC(=O)[C@H]1CCc2ccccc2C1c1ccccc1</smiles>
$\mathbf{4} \mathbf{c}$ monitored by TLC. Upon consumption of $\mathbf{4} \mathbf{c}$, DBU (50 $\mu \mathrm{L}, 0.33 \mathrm{mmol})$ and $\mathrm{MeOH}(1 \mathrm{~mL})$ add to the solution. After $1 \mathrm{~h}$ the reaction mixture was quenched by $\mathrm{HCl}$ aq, and solids were removed by filtration. The filtrate was extracted with dichloromethane. The extracts were dried with $\mathrm{Na}_{2} \mathrm{SO}_{4}$, and concentrated in vacuo. The crude mixture was purified by column chromatography $\left(\mathrm{SiO}_{2}\right.$, eluent: $15 \%$ ethyl acetate in hexane) to give 5 ( $22 \mathrm{mg}, 0.083 \mathrm{mmol}, 83 \%$ yield) as a colorless oil; Rf $\mathbf{5}: 0.35$ (10\% ethyl acetate in hexane); ${ }^{1} \mathrm{H}$ NMR $\left(600 \mathrm{MHz}, \mathrm{CDCl}_{3}\right) \delta 7.27(\mathrm{t}, J=7.6 \mathrm{~Hz}, 2 \mathrm{H}), 7.20(\mathrm{~m}, 1 \mathrm{H})$, 7.12-7.09 (m, 4H), $7.02(\mathrm{~m}, 1 \mathrm{H}), 6.77(\mathrm{~d}, J=7.6 \mathrm{~Hz}, 1 \mathrm{H}), 4.43(\mathrm{~d}, J=9.0 \mathrm{~Hz}, 1 \mathrm{H}), 3.55(\mathrm{~s}, 3 \mathrm{H})$, $3.00(\mathrm{~m}, 1 \mathrm{H}), 2.95-2.89(\mathrm{~m}, 2 \mathrm{H}), 2.16(\mathrm{~m}, 1 \mathrm{H}), 2.03(\mathrm{~m}, 1 \mathrm{H}) ;{ }^{13} \mathrm{C}\left\{{ }^{1} \mathrm{H}\right\} \mathrm{NMR}\left(150 \mathrm{MHz}, \mathrm{CDCl}_{3}\right) \delta$ 175.2, 144.8, 138.2, 135.9, 130.0, 129.1, 128.6, 128.4, 126.5, 126.0, 126.0, 51.6, 49.3, 47.8, 28.7, 25.7; HRMS (ESI-TOF) $m / z$ calcd for $\mathrm{C}_{18} \mathrm{H}_{18} \mathrm{NaO}_{2}(\mathrm{M}+\mathrm{Na})^{+} 289.1204$, found 289.1208 . 
Mechanistic investigation: Examination of a reaction pathway via the aldol condensation product. As a preliminary examination for the reaction pathway, we subjected the aldol condensation product to the acidic reaction conditions for the $[5+1]$ annulation as follows. Enone 6 was separately prepared by the base-catalyzed aldol reaction, and treated with $\mathrm{BF}_{3} \cdot \mathrm{OEt}_{2}$. The Nazarov cyclization product, 7, was obtained instead of the tetraline product, 4ae. This suggests that the conventional aldol condensation is not involved in the reaction pathway of the [5+1] annulation.

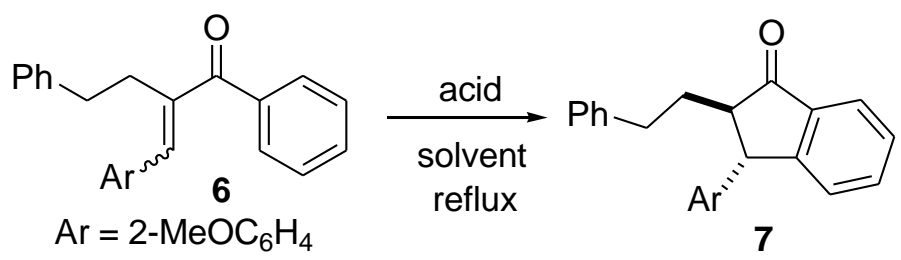

Enone 6 was prepared by aldol condensation reaction of 3a with 2-methoxyabenzaldehyde as follows. To a stirred solution of 3a (673 mg, $3 \mathrm{mmol})$ and 2-methoxybenzaldehyde (1.23 g, 4.5 $\mathrm{mmol})$ in ethanol $(5 \mathrm{~mL})$ was added $\mathrm{NaOH}(2.4 \mathrm{~g} 6 \mathrm{mmol})$. The mixture was refluxed with an oil bath for $12 \mathrm{~h}$. The reaction mixture was then quenched by $\mathrm{HCl}$ aqueous solution, and extracted by dichloromethane. The extracts were dried with $\mathrm{Na}_{2} \mathrm{SO}_{4}$, and concentrated in vacuo. The crude mixture was purified by $\mathrm{SiO}_{2}$ column (eluent: 5\% ethyl acetate in hexane) to give 6 (294 mg, 29\% yield) as a mixture of $E / Z$ isomers (8 : 2). Rf: 0.45 (10\% ethyl acetate in hexane); ${ }^{1} \mathrm{H}$ NMR (600 $\left.\mathrm{MHz}, \mathrm{CDCl}_{3}\right)$ of the major isomer $\delta 7.78(\mathrm{~d}, J=7.6 \mathrm{~Hz}, 2 \mathrm{H}), 7.53(\mathrm{t}, J=7.6 \mathrm{~Hz}, 1 \mathrm{H}), 7.43(\mathrm{t}, J=7.6$ $\mathrm{Hz}, 2 \mathrm{H}), 7.29$ (t, $J=7.6 \mathrm{~Hz}, 1 \mathrm{H}), 7.25$ (s, 1H), 7.23-7.20 (m,2H), 7.18 (t, $J=7.6 \mathrm{~Hz}, 2 \mathrm{H}), 7.14$ (d, $J$ $=7.6 \mathrm{~Hz}, 2 \mathrm{H}), 6.94(\mathrm{t}, J=7.6 \mathrm{~Hz}, 1 \mathrm{H}), 6.85(\mathrm{~d}, J=7.6 \mathrm{~Hz}, 1 \mathrm{H}), 3.74(\mathrm{~s}, 3 \mathrm{H}) 2.95(\mathrm{t}, J=7.6 \mathrm{~Hz}, 2 \mathrm{H})$, $2.82(\mathrm{t}, J=7.6 \mathrm{~Hz}, 2 \mathrm{H})$; selected ${ }^{1} \mathrm{H} \mathrm{NMR}\left(600 \mathrm{MHz}, \mathrm{CDCl}_{3}\right)$ of the minor isomer $\delta 7.34(\mathrm{t}, J=7.6$ Hz, 1H), 7.00 (t, $J=7.6 \mathrm{~Hz}, 1 \mathrm{H}), 6.63(\mathrm{~d}, J=7.6 \mathrm{~Hz}, 1 \mathrm{H}), 6.58$ (t, $J=7.6 \mathrm{~Hz}, 1 \mathrm{H}), 3.75(\mathrm{~s}, 3 \mathrm{H})$, 2.88-2.86 (m, 2H); ${ }^{13} \mathrm{C}\left\{{ }^{1} \mathrm{H}\right\} \mathrm{NMR}\left(150 \mathrm{MHz}, \mathrm{CDCl}_{3}\right)$ of the mixture $\delta 200.3,199.0,157.2,156.4$, 141.6, 141.3, 140.5, 140.0, 138.72, 138.67, 136.4, 132.7, 131.8, 130.2, 129.92, 129.88, 129.4, 129.2, 129.0, 128.5, 128.30, 128.28, 128.1, 127.6, 125.93, 125.88, 125.2, 124.6, 120.2, 120.1, 110.5, 110.0, $55.4,55.1,38.1,34.7,34.4,29.7$; HRMS (ESI-TOF) $\mathrm{m} / z$ calcd for $\mathrm{C}_{17} \mathrm{H}_{15} \mathrm{~F}_{3} \mathrm{NaO}(\mathrm{M}+\mathrm{Na})^{+}$ 365.1517 , found 365.1514 .

To a stirred solution of $\mathbf{6}(76.6 \mathrm{mg}, 0.224 \mathrm{mmol})$ in a mixed solvent of hexane and dichloromethane (9: $1(\mathrm{v} / \mathrm{v}), 2 \mathrm{~mL})$ was added $\mathrm{BF}_{3} \cdot \mathrm{OEt}_{2}(30 \mu \mathrm{L}, 0.269 \mathrm{mmol})$. The mixture was refluxed with an oil bath for $12 \mathrm{~h}$. The reaction mixture was then quenched by $\mathrm{Na}_{2} \mathrm{CO}_{3}$ aqueous solution, and extracted by dichloromethane. The extracts were dried with $\mathrm{Na}_{2} \mathrm{SO}_{4}$, and concentrated in vacuo. The crude mixture was purified by $\mathrm{SiO}_{2}$ column (eluent: $5 \%$ ethyl acetate in hexane) to give 7 (66.9 $\mathrm{mg}, 87 \%$ yield) as an oil. Rf: 0.34 (10\% ethyl acetate in hexane), ${ }^{1} \mathrm{H}$ NMR $\left(600 \mathrm{MHz}, \mathrm{CDCl}_{3}\right) \delta 7.76(\mathrm{~d}, J=$ $7.6 \mathrm{~Hz}, 1 \mathrm{H}), 7.50(\mathrm{t}, J=7.6 \mathrm{~Hz}, 1 \mathrm{H}), 7.35(\mathrm{t}, J=7.6 \mathrm{~Hz}, 1 \mathrm{H}), 7.23(\mathrm{t}, J=7.6 \mathrm{~Hz}, 1 \mathrm{H}), 7.18(\mathrm{t}, J=$ 
$7.6 \mathrm{~Hz}, 2 \mathrm{H}), 7.17(\mathrm{~d}, J=7.6 \mathrm{~Hz}, 1 \mathrm{H}), 7.11(\mathrm{t}, J=7.6 \mathrm{~Hz}, 1 \mathrm{H}), 7.04(\mathrm{~d}, J=7.6 \mathrm{~Hz}, 2 \mathrm{H}), 6,94(\mathrm{~d}, J=$ $7.6 \mathrm{~Hz}, 1 \mathrm{H}), 6.89$ (d, $J=7.6 \mathrm{~Hz}, 1 \mathrm{H}), 6.87$ (t, $J=7.6 \mathrm{~Hz}, 1 \mathrm{H}), 4.63$ (s, 1H), 3.71 (s, 3H), 2.77-2.71 (m, 3H), $2.25(\mathrm{~m}, 1 \mathrm{H}), 2.00(\mathrm{~m}, 1 \mathrm{H}) ;{ }^{13} \mathrm{C}\left\{{ }^{1} \mathrm{H}\right\} \mathrm{NMR}\left(150 \mathrm{MHz}, \mathrm{CDCl}_{3}\right) \delta 208.0,157.5,157.1,141.9$, 136.6, 134.7, 131.7, 129.2, 128.5, 128.2, 128.1, 127.4, 126.2, 125.7, 123.4, 121.0, 111.2, 56.0, 55.4, 46.3, 33.2, 33.1; HRMS (ESI-TOF) $\mathrm{m} / z$ calcd for $\mathrm{C}_{17} \mathrm{H}_{15} \mathrm{~F}_{3} \mathrm{NaO}(\mathrm{M}+\mathrm{Na})^{+} 365.1517$, found 365.1524 .

Theoretical Calculations. All geometrical parameters of the reaction intermediates and the transition states were fully optimized at the level of the hybrid exchange-correlation functional $\mathrm{B}^{2} \mathrm{LYP}^{\mathrm{S} 5}$ with the 6-31G(d) basis set. All calculations were carried out using the Gaussian 09, Revision C.01 package. ${ }^{\mathrm{S} 6}$

(S5) (a) Becke, A. D. J. Chem. Phys. 1993, 98, 1372-1377. (b) Becke, A. D. J. Chem. Phys. 1993, 98, 5648-5652. (c) Becke, A. D. Phys. Rev. 1998, A38, 3098-3100.

(S6) Frisch, M. J.; Trucks, G. W.; Schlegel, H. B.; Scuseria, G. E.; Robb, M. A.; Cheeseman, J. R.; Scalmani, G.; Barone, V.; Mennucci, B.; Petersson, G. A.; Nakatsuji, H.; Caricato, M.; Li, X.; Hratchian, H. P.; Izmaylov, A. F.; Bloino, J.; Zheng, G.; Sonnenberg, J. L.; Hada, M.; Ehara, M.; Toyota, K.; Fukuda, R.; Hasegawa, J.; Ishida, M.; Nakajima, T.; Honda, Y.; Kitao, O.; Nakai, H.; Vreven, T.; Montgomery, Jr., J. A.; Peralta, J. E.; Ogliaro, F.; Bearpark, M.; Heyd, J. J.; Brothers, E.; Kudin, K. N.; Staroverov, V. N.; Keith, T.; Kobayashi, R.; Normand, J.; Raghavachari, K.; Rendell, A.; Burant, J. C.; Iyengar, S. S.; Tomasi, J.; Cossi, M.; Rega, N.; Millam, J. M.; Klene, M.; Knox, J. E.; Cross, J. B.; Bakken, V.; Adamo, C.; Jaramillo, J.; Gomperts, R.; Stratmann, R. E.; Yazyev, O.; Austin, A. J.; Cammi, R.; Pomelli, C.; Ochterski, J. W.; Martin, R. L.; Morokuma, K.; Zakrzewski, V. G.; Voth, G. A.; Salvador, P.; Dannenberg, J. J.; Dapprich, S.; Daniels, A. D.; Farkas, O.; Foresman, J. B.; Ortiz, J. V.; Cioslowski, J.; Fox, D. J.; Gaussian, Inc., Wallingford CT, 2010. 


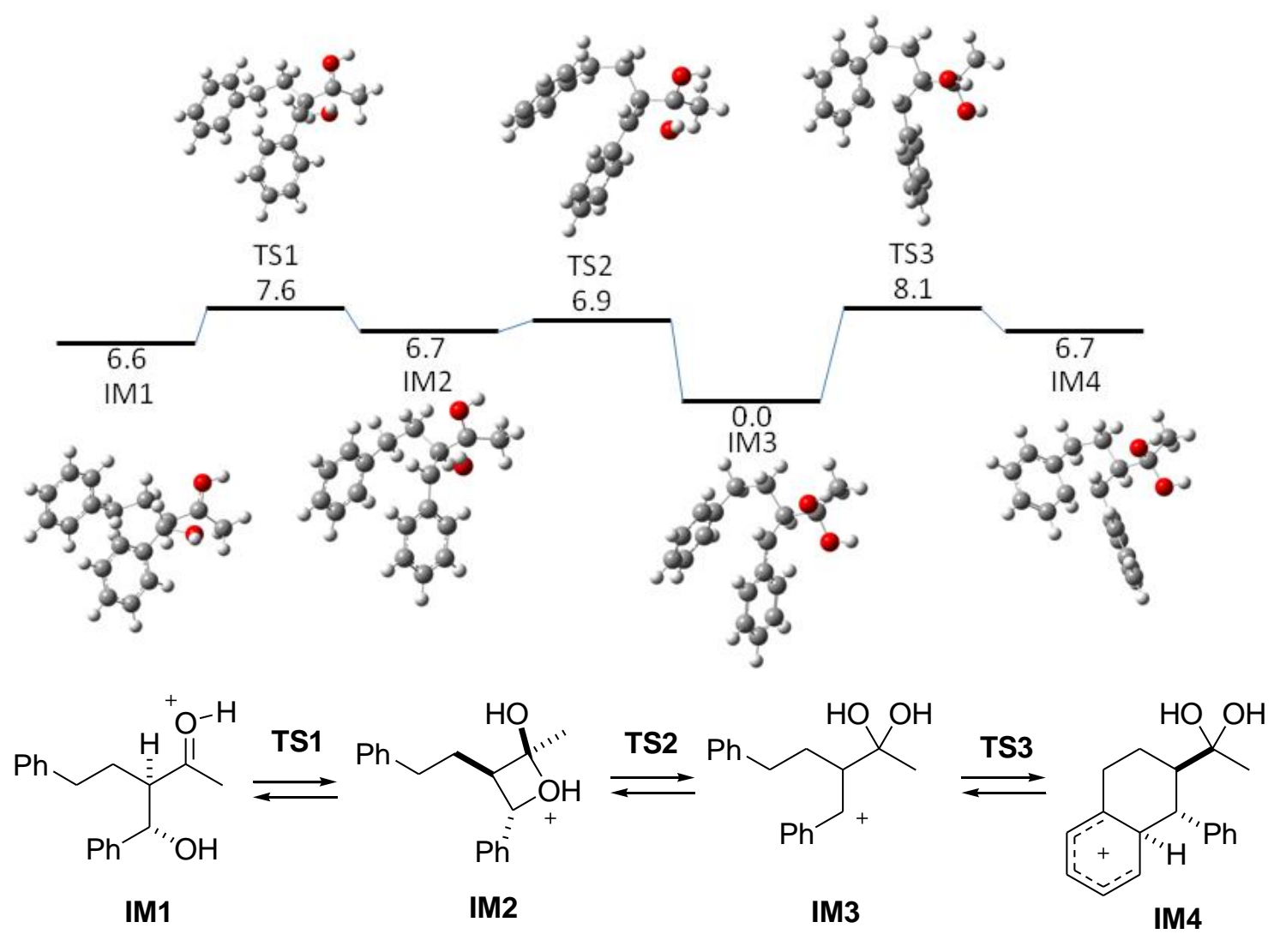

Scheme S1. Energy profile (in $\mathrm{kcal} / \mathrm{mol}$ ) for formation of trans tetraline product 




Scheme S2. Comparison of energy profiles (in $\mathrm{kcal} / \mathrm{mol}$ ) for the trans tetraline formation and Grob elimination 

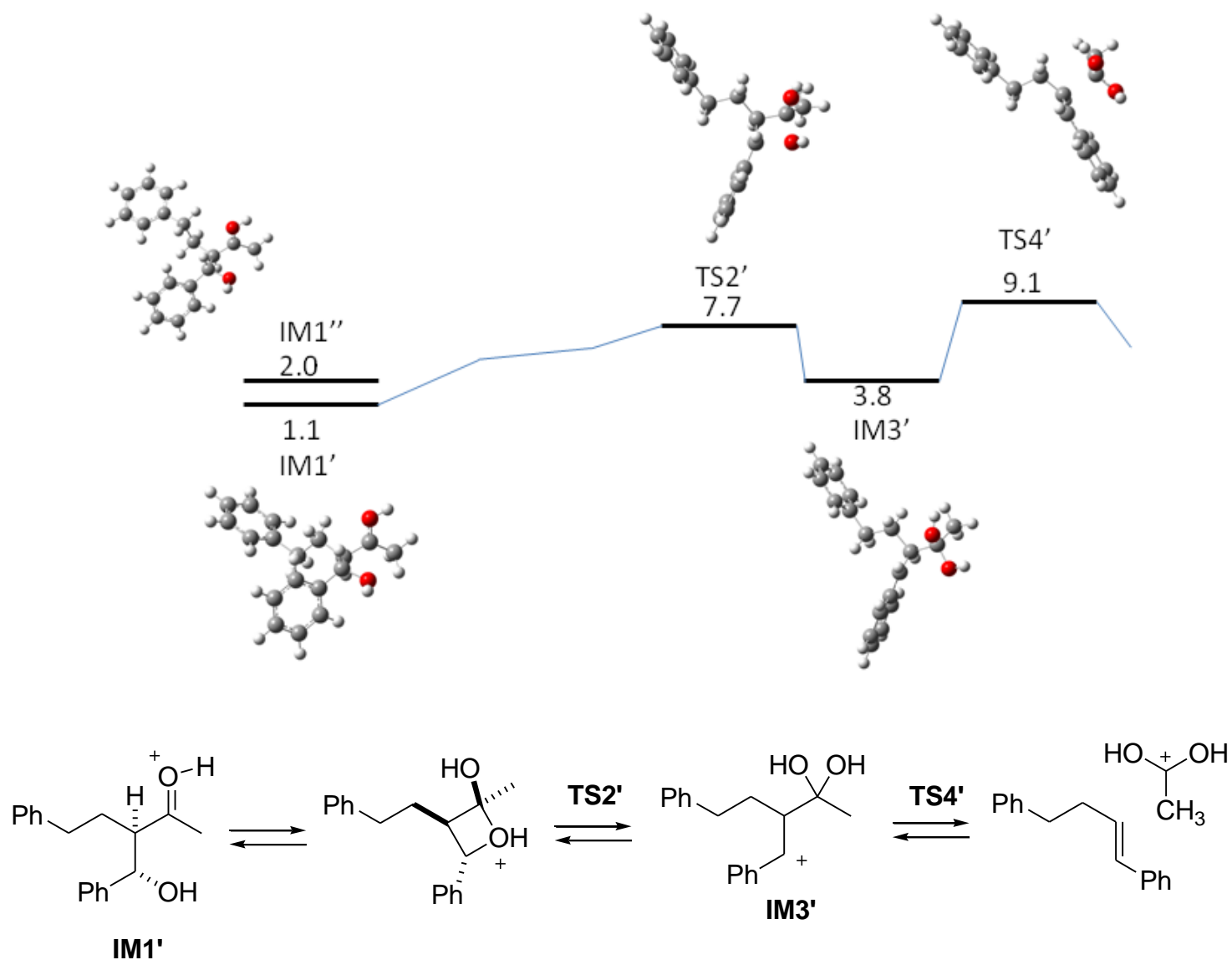

(a conformer of IM1)

Scheme S3. Energy profile (in $\mathrm{kcal} / \mathrm{mol}$ ) for Grob elimination 

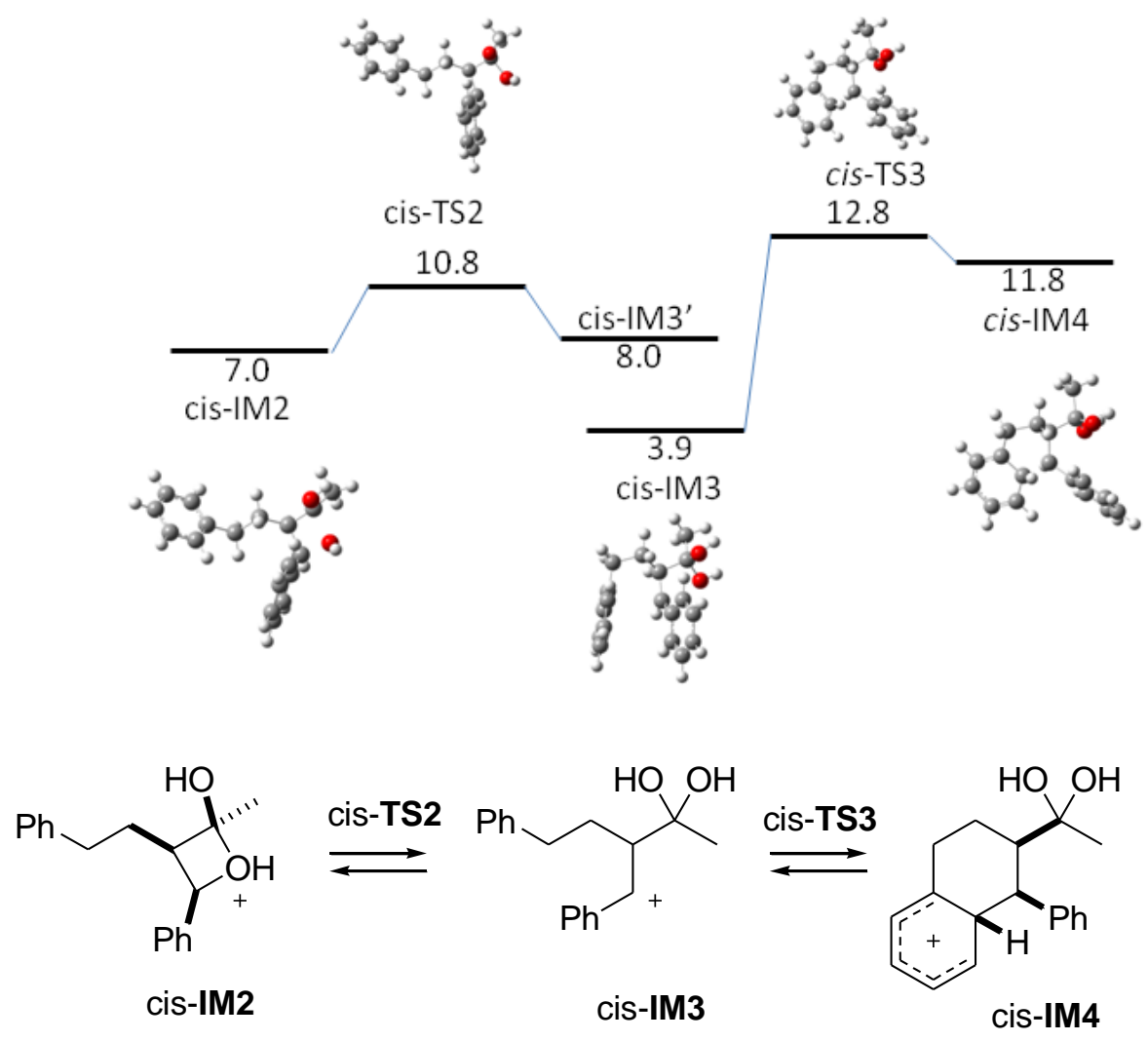

Scheme S4. Energy profile (in $\mathrm{kcal} / \mathrm{mol}$ ) for formation of cis tetraline product 


\section{IM1}

Calculation Method $=$ RB3LYP

Basis Set $=6-31 \mathrm{G}(\mathrm{d})$

Charge $=1$

Spin $=$ Singlet

$\mathrm{E}(\mathrm{RB} 3 \mathrm{LYP})=-848.73993792$ a.u.

RMS Gradient Norm $=0.00000505$ a.u.

Imaginary Freq =

Dipole Moment $=10.9202$ Debye

11

$\mathrm{C}$

$\mathrm{H}$

1.09810100

$-1.80647100$

0.80201000

0.96433700

$-2.39812700$

$-0.10787300$

$\mathrm{H}$

1.76356600

$-2.39431100$

$-0.50255700$

1.44658000

1.86551200

$\mathrm{H}$

1.96316000

0.13219700

0.44244700

C

3.24808400

$-0.84047700$

1.32906100

C

1.28418400

0.38166100

$-0.01131800$

C

0.26205700

0.59926200

1.42059400

$-0.72651700$

2.46146600

$-0.35347300$

$-0.99642800$

1.42409100

0.52897600

C

$-0.31175900$

3.47848100

$-0.96797300$

C

1.58433700

2.48887800

0.79862000

$-1.90501700$

2.44959000

0.98839300

C

$-1.27192200$

0.61993900

$-0.70445800$

$\mathrm{H}$

$-1.56612500$

3.47445300

$-1.64462900$

0.17923300

$-0.04361700$

4.27897600

2.44311600

1.48168900

$-2.87699000$

$-2.27429600$

4.27175600

$-1.18773400$

0.38503000

2.48811000

1.00978700

$-1.22759700$

3.33710100

$-1.79144400$

$-0.87835200$

4.25692500

$-1.96631500$

$-1.16589700$

4.46122200

$-0.21742100$

0.56096400

4.54363500

$-0.52966400$

1.61285000

5.37938400

$-0.49180800$

0.03258200

4.33532000

0.86928000

0.55433500

2.25014600

1.61919900

$-1.94785400$

0.87758100

$-0.30247600$

$-1.48187200$

$-0.24120400$

$-1.63493400$

1.55296900

$-0.17607200$

$-0.77931100$

2.23629900

$-0.34215400$

$-2.51603400$

2.20138100

$-1.50849100$

$-1.52035000$

0.71994200

$-2.55501000$

$-0.70603600$

1.17270000

$-1.71216100$

$-2.27784400$

$-0.44196300$

$-3.76546000$

$-0.64025500$

0.48290600

$-2.42104200$

$-0.11620600$

2.07649800

$-2.91801700$

$-2.20538300$

$-1.14332800$

$-0.94022900$

$-2.95345400$

$-0.80299800$

$-3.94932500$

$-1.38490400$

$-0.68369300$

$-4.56424700$

$-0.00592100$

0.85690900

$-3.05533800$

$-2.80311800$

$-2.04020400$

$-4.89072100$

$-1.33556100$

$-1.22320500$ 


\section{IM1'}

Calculation Method = RB3LYP

Basis Set $=6-31 \mathrm{G}(\mathrm{d})$

Charge $=1$

Spin $=$ Singlet

$\mathrm{E}(\mathrm{RB} 3 \mathrm{LYP})=-848.74426356$ a.u.

RMS Gradient Norm $=0.00000305$ a.u.

Imaginary Freq $=$

Dipole Moment $=6.8061$ Debye 11

C

$\mathrm{H}$

0.82290200

$\begin{array}{ll}0.84430800 & 0.01588500\end{array}$

$\begin{array}{lll}1.02427100 & 1.06408900 & -1.03762700\end{array}$

$\mathrm{H}$

1.41816700

$1.54558700 \quad 0.61169600$

C

$-0.68093100$

1.14687300

0.32206400

$\mathrm{H}$

$-0.87509100$

0.96619200

1.38289500

$-1.05616000$

2.64200300

0.00094600

$-1.55644500$

0.33519300

$-0.54059100$

C

$-2.39270600$

$-0.73510400$

$-0.20943300$

$-2.55564400$

$-1.21385900$

1.12807500

$-3.11976200$

$-1.37100700$

$-1.26520600$

$-3.39934800$

$-2.27612100$

1.38377400

$-2.01970800$

$-0.73865500$

1.94165800

$-3.96101700$

$-2.43411900$

$-0.99690000$

$-3.00097600$

$-1.00443300$

$-2.28088400$

$-4.09871600$

$-2.88436200$

0.32527200

$-3.52794600$

$-2.64254300$

$-4.51210400$

$-2.91683400$

2.39684100

$-4.76115000$

$-3.71907200$

$-1.79702300$

$-2.46511100$

2.62353300

0.53730400

$-0.75437600$

2.93233400

0.11896700

0.04872900

3.47397800

$-1.35066600$

$-0.42161100$

3.64778400

$-1.40132300$

0.67099900

3.66123900

0.95699000

$-0.79092500$

4.65174600

0.88344900

$-0.69775100$

3.42141100

0.71750800

$-2.80400600$

3.48099500

1.99015100

$\mathrm{H}$

$-1.52137100$

0.61963200

$-0.19426100$

1.26253500

$-0.59322100$

$-1.59275200$

0.99734900

$-0.82518600$

0.34928900

0.71281500

$-1.30389800$

1.38903200

2.75295900

$-0.76901700$

$-0.28313300$

3.63816300

$-0.65898600$

0.14934200

3.27694600

$-1.00491500$

1.23021100

5.01481100

$-0.77954100$

$-1.12993800$

3.24707300

$-0.48628000$

1.03844900

4.65215700

$-1.12705400$

2.23086100

2.60299500

$-1.32470100$

$\mathrm{H}$

$-1.10595000$

$-1.97868700$

5.52491700

$-1.01255900$

$-0.24003900$

$\mathrm{H}$

5.68763400

$-0.69777200$

1.88730200 


$\begin{array}{llll}\mathrm{H} & 5.04253000 & -1.31780600 & -2.32032700 \\ \mathrm{H} & 6.59605700 & -1.11116400 & -0.39002200\end{array}$

\section{IM1"}

Calculation Method $=$ RB3LYP

Basis Set $=6-31 \mathrm{G}(\mathrm{d})$

Charge $=1$

Spin $=$ Singlet

$\mathrm{E}(\mathrm{RB} 3 \mathrm{LYP})=-848.74713035$ a.u.

RMS Gradient Norm $=0.00000581$ a.u.

Imaginary Freq =

Dipole Moment $=9.8520$ Debye

11

C

$\mathrm{H}$

C

$\mathrm{H}$

C

C

$\mathrm{H}$

C

$\mathrm{C}$

C

C

$\mathrm{H}$

$\mathrm{C}$

$\mathrm{H}$

C

$\mathrm{H}$

$\mathrm{H}$

$\mathrm{H}$

$\mathrm{O}$

$\mathrm{O}$

$\mathrm{H}$

C

$\mathrm{H}$

$\mathrm{H}$

$\mathrm{H}$

$\mathrm{H}$

$\mathrm{H}$

C

$\mathrm{H}$

$\mathrm{H}$

C

C

C

C

$\mathrm{H}$

C

$\mathrm{H}$

C
$-0.41405000$

$-0.90022900$

1.04400000

1.54842600

1.14783100

1.92123100

1.42043700

2.14048800

1.50517000

2.99338300

1.71227400

0.86247100

3.20072000

3.50807200

2.55755800

1.22402600

3.86595300

2.72139600

3.12994600

0.59278800

0.72413200

1.87223700

1.48431900

1.82861300

2.91767000

$-0.29016000$

3.78982700

$-1.33684000$

$-1.50236500$

$-0.86378900$

$-2.67178600$

$-2.80306400$

$-3.78606300$

$-4.02091300$

$-1.94832500$

$-5.00634200$

$-3.70368500$

$-5.12586600$
$-0.75568800$

$-1.30641900$

$-1.29536600$

$-1.14944200$

$-2.73666100$

$-0.54514000$

$-0.63911800$

0.90615800

1.90868900

1.26932400

3.25368300

1.63834200

2.61320800

0.49893000

3.60651800

4.02388100

2.88740800

4.65347400

$-1.31537500$

$-3.13313700$

$-4.08806600$

$-3.68712300$

$-3.62109000$

$-4.72353600$

$-3.34961300$

0.27102100

$-0.86121400$

$-0.75231600$

$-1.77317900$

$-0.20134500$

$-0.11586300$

1.27877000

$-0.90875600$

1.86672900

1.90997800

$-0.32358500$

$-1.99307700$

1.06601800
0.61452400

1.42863800

0.46165600

1.42209700

0.14732100

$-0.61073000$

$-1.58273400$

$-0.27646200$

$-1.02342600$

0.77855800

$-0.71671000$

$-1.85806000$

1.08165200

1.34668100

0.33725800

$-1.30614300$

1.89494100

0.57445300

$-0.60405900$

$-0.94910300$

$-1.12463100$

1.01684600

2.04129300

0.66918200

1.05413000

0.97697500

$-1.15521800$

$-0.61931700$

$-0.97897500$

$-1.44057500$

$-0.27846500$

$-0.22788800$

0.02142500

0.11292900

$-0.46398800$

0.36273200

$-0.02235900$

0.41005100 


$\begin{array}{cccc}\mathrm{H} & -4.10927400 & 2.94908700 & 0.14100100 \\ \mathrm{H} & -5.86354100 & -0.95266400 & 0.58520200 \\ \mathrm{H} & -6.07565800 & 1.52315100 & 0.67166400\end{array}$

\section{TS1}

Calculation Method $=$ RB3LYP

Basis Set $=6-31 \mathrm{G}(\mathrm{d})$

Charge $=1$

Spin $=$ Singlet

$\mathrm{E}(\mathrm{RB} 3 \mathrm{LYP})=-848.73829934$ a.u.

RMS Gradient Norm $=0.00000359$ a.u.

Imaginary Freq $=1$

Dipole Moment $=10.2650$ Debye

11

C

$\mathrm{H}$

$$
1.20755700
$$

$-1.90493900$

0.84850400

$\mathrm{H}$

1.15888600

$-2.51776400$

$-0.05682600$

$\mathrm{C}$

1.94652700

$-2.38810700$

1.50068900

1.76411700

$-0.51190100$

0.49198200

$\mathrm{H}$

1.83966200

0.09763000

1.39866400

C

3.15534800

$-0.58260500$

$-0.13214000$

$\begin{array}{ll}\mathrm{C} & 1.07885000\end{array}$

0.31346700

$-0.62770000$

0.13473700

1.42622600

$-0.30161600$

0.49129900

2.43114000

0.61242500

C

$-1.09512100$

1.50878200

$-0.96676700$

C $\quad-0.37135100$

3.49502300

0.85881700

1.45178300

2.39593000

1.12081300

C

$-1.95937000$

2.57500000

$-0.71833900$

$0.72827100-1.66374700$

$-1.38815500$

3.56802100

0.19268000

$-1.59906600$

4.26941300

1.56512500

$-2.91239000$

2.62701200

$-1.23559800$

$\mathrm{H}$

$-2.27090800$

4.39924500

0.38537600

2.36130500

0.87173400

$-1.18002800$

O

3.33891900

$-1.59193200$

$-0.94492900$

4.26014400

$-1.64824400$

$-1.26661000$

4.34325400

0.09303600

0.46210100

4.67153700

$-0.51228800$

1.31996400

5.17342300

0.16614900

$-0.24889900$

4.08851600

1.08935200

0.82383200

2.33079100

0.94246200

$-2.15360700$

0.64949300

$-0.36203000$

$-1.37208800$

$-0.14879900$

$-1.92027000$

1.58188100

$-0.12882800$

$-1.19461000$

2.40446700

$-0.22973600$

$-2.90558600$

2.06071900

$-1.38966200$

$-1.69231400$

0.73428100

$-2.42151400$

$-0.87499800$

1.21291800

$-1.57614400$

$-2.34865600$

$-0.49210100$

$-3.60320000$

$-0.71030700$

0.48924700

$-2.30169800$

$-0.36467800$

2.16569900

$-2.75247900$

$-2.17811800$

$-1.22507300$

$\mathrm{H}$

$-0.81392200$

$-3.02472800$

$-0.87371600$ 


$\begin{array}{lrrr}\mathrm{C} & -3.77097600 & -1.35700000 & -0.73629100 \\ \mathrm{H} & -4.39255800 & -0.07728400 & 0.88467900 \\ \mathrm{H} & -2.88031700 & -2.70236000 & -2.16825000 \\ \mathrm{H} & -4.69131400 & -1.23371300 & -1.29988500\end{array}$

\section{IM2}

Calculation Method $=$ RB3LYP

Basis Set $=6-31 \mathrm{G}(\mathrm{d})$

Charge $=1$

Spin $=$ Singlet

$\mathrm{E}(\mathrm{RB} 3 \mathrm{LYP})=-848.73971640$ a.u.

RMS Gradient Norm $=0.00000248$ a.u.

Imaginary Freq =

Dipole Moment $=8.3079$ Debye

11

$\mathrm{C}$

$\mathrm{H}$

1.32772100

$-1.99790100$

0.74196400

$-2.53347900$

$-0.21268000$

$\mathrm{H}$

1.32437400

$-2.43935200$

1.33303400

C

2.13980400

$-0.52587700$

0.50003700

$\mathrm{H}$

1.69078500

0.01039200

1.45347000

C

1.69889100

$-0.29568700$

$-0.21976500$

0.88544400

0.25962600

$-0.55226000$

0.01001700

1.40802600

$-0.24861600$

$2.29325300 \quad 0.80957000$

$\begin{array}{lr}\mathrm{C} & 0.28176500\end{array}$

1.64230400

$-1.07322300$

$-1.10272700$

3.38870100

1.03529400

C

$-0.54482500$

2.13715000

1.45392700

1.14241900

2.73652300

$-0.84093100$

C

$-1.93271700$

0.95207500

$-1.88073200$

$-1.33171700$

3.61103100

0.21044700

$-0.32884100$

4.07017500

1.85228400

$-2.79568800$

2.90515900

$-1.47740700$

$-2.29754400$

4.46600100

0.39098500

2.29361200

0.80099500

$-1.16223000$

3.40252400

$-1.33595400$

$-1.00662000$

4.34644600

$-1.28519100$

$-1.24130600$

4.15877200

4.57197900

0.42254100

0.48489200

$-0.24184300$

0.69332400

1.25260900

4.95834400

3.80004900

1.33334600

$-0.21458900$

2.39637400

0.58108200

0.96775100

0.47947600

$-0.38257100$

$-2.11227800$

$-0.00057700$

$-2.22610400$

$-1.33378500$

0.00840900

$-1.66315900$

1.48992200

$-0.02783200$

$-3.28606500$

2.43159700

$-1.25781600$

$-1.88703500$

1.77467300

$-2.22705400$

$-1.03368800$

0.70819200

$-1.50277600$

$-2.45890500$

1.25043600

C

$-3.40600200$

$-0.75240000$

$-0.55128800$

$\mathrm{C}$
$\mathrm{H}$

$-2.06273700$

$-0.59202500$

0.55720100

2.23065000 


$\begin{array}{lccc}\mathrm{C} & -2.67631500 & -2.17421100 & -1.25120000 \\ \mathrm{H} & -0.78708100 & -3.15820800 & -0.97935700 \\ \mathrm{C} & -3.63235100 & -1.31800500 & -0.69825800 \\ \mathrm{H} & -4.14833300 & -0.09539900 & 1.00148400 \\ \mathrm{H} & -2.85400700 & -2.63746300 & -2.21796400 \\ \mathrm{H} & -4.55235400 & -1.10742100 & -1.23605100\end{array}$

\section{TS2}

Calculation Method $=$ RB3LYP

Basis Set $=6-31 \mathrm{G}(\mathrm{d})$

Charge $=1$

Spin $=$ Singlet

$\mathrm{E}(\mathrm{RB} 3 \mathrm{LYP})=-848.73938596$ a.u.

RMS Gradient Norm $=0.00000777$ a.u.

Imaginary Freq $=1$

Dipole Moment $=6.9178$ Debye

11

C

$\mathrm{H}$

$$
1.50324700
$$

$-1.90114200$

0.70509000

1.55007000

$-2.41356200$

$-0.26055300$

$\mathrm{H}$

2.35268100

$-2.27806800$

1.28830500

1.73235300

$-0.39676500$

0.49109800

$\mathrm{H}$

1.70392400

3.04800100

0.11721900

1.45631000

C

$-0.02675100$

$-0.23476400$

$\begin{array}{lr}\mathrm{C} & 0.82203400\end{array}$

0.31437500

$-0.14275900$

1.35715300

2.16307400

$-0.51122100$

$-0.21973600$

C $\quad-0.06275900$

1.58627300

$-1.17886500$

3.16715500

0.93579200

$-1.14896500$

C

$-0.99691900$

2.01322100

1.15129900

0.73316800

$-2.11604200$

2.58861800

1.65843900

C

$-1.25607800$

0.95837200

$-0.92537900$

$\mathrm{H}$

$-2.02331600$

3.38023500

$-2.03214100$

$-0.93080300$

3.78743700

0.22215400

$\mathrm{H}$

$-2.91601300$

2.75354300

2.03958000

$-2.75219600$

4.16626400

$-1.64000600$

$\mathrm{H}$

2.35078900

1.07976600

0.39672000

3.44009500

$-1.01359600$

4.39314700

$-0.94884900$

$-1.06105000$

$\mathrm{O}$

$\mathrm{H}$

4.16731100

0.59934800

$-1.10585800$

$-1.28885500$

4.58045300

$-0.14331900$

0.94896600

0.56071400

4.96795000

3.80687800

1.45375800

1.25202200

$-0.10215900$

2.53156200

0.92910200

1.13735800

0.58381200

$-0.28564400$

$-2.01216600$

0.19933000

$-2.25969500$

$-1.38762700$

C

0.17195800

$-1.74661200$

1.44217900

0.24273300

$-3.33252100$

2.41148400

$\mathrm{H}$

$-1.07681700$

$-1.96125900$

1.67168300

C

$-2.10746200$

$-1.21764300$

0.67516200

C

$-1.27602800$

$-2.46197900$

1.26539000

$-0.62271900$ 


$\begin{array}{lrrr}\mathrm{C} & -3.29863100 & -0.97066500 & 0.58126600 \\ \mathrm{H} & -1.98005700 & -0.83636200 & 2.27599300 \\ \mathrm{C} & -2.46353800 & -2.21247600 & -1.31268300 \\ \mathrm{H} & -0.51159500 & -3.07942600 & -1.09011600 \\ \mathrm{C} & -3.47879100 & -1.46329800 & -0.71260100 \\ \mathrm{H} & -4.08774000 & -0.39923600 & 1.06182000 \\ \mathrm{H} & -2.60438100 & -2.62078100 & -2.30981400 \\ \mathrm{H} & -4.40863600 & -1.28020500 & -1.24348000\end{array}$

\section{TS2'}

Calculation Method $=$ RB3LYP

Basis Set $=6-31 \mathrm{G}(\mathrm{d})$

Charge $=1$

Spin $=$ Singlet

$\mathrm{E}(\mathrm{RB} 3 \mathrm{LYP})=-848.73811476$ a.u.

RMS Gradient Norm $=0.00000252$ a.u.

Imaginary Freq $=1$

Dipole Moment $=8.7818$ Debye

11

C

$\mathrm{H}$

$\mathrm{H}$

$\mathrm{C}$

$\mathrm{H}$

C

0.87337400

0.95634300

0.04872900

1.09746900

1.14333600

$-1.00667400$

1.43891200

1.70307900

0.61968600

$-0.62223200$

1.19222100

0.30960700

1.00430000

1.36391900

$-1.18940400$

2.57854800

$-0.06513900$

0.39957000

$-0.57206400$

C $\quad-2.36675700$

$-0.73872400$

$-0.20400300$

C

$-2.65777200$

$-1.06465300$

1.14200600

C

$-2.86215900$

$-1.56969700$

$-1.23784400$

$-3.41167100$

$-2.19085300$

1.43682900

$-2.29918500$

$-0.43491000$

1.94930100

C

$-3.60999600$

$-2.69928300$

$-0.93584800$

$-2.64178300$

$-1.32455400$

$-2.27372800$

$-3.88484700$

$-3.00797900$

0.40097500

$\mathrm{H}$

$-3.63400400$

$-2.44052700$

2.46925700

$-3.97860900$

$-3.33873600$

$-1.73129000$

$-4.47108400$

$-3.89066200$

0.63937800

$\mathrm{O}$

$-2.55118500$

2.00008300

$-0.46113100$

$\mathrm{O}$

$-0.59044500$

3.06014800

$-1.21026900$

$\mathrm{H}$

$-0.63975400$

4.03055100

3.60469500

$-1.23910600$

$-1.37900800$

3.92307100

1.02610400

$-0.40107400$

4.48335400

1.40311300

$-1.91198800$

3.19149600

0.64311300

$-1.95827600$

2.36827600

1.85447000

$-2.80829600$

0.46432600

$-1.33113300$

$-1.33005800$

$-0.45520700$

$-1.63270600$

1.35059100

0.45007600

1.11160300

$-0.63071800$

1.50732600

$\mathrm{H}$

0.79449100

$-1.20850000$

$-0.12425000$

2.83760200

$-0.63423800$

0.22148600

C

3.75660200

$-0.33327900$

1.23485900 


$\begin{array}{lccc}\mathrm{C} & 3.32242900 & -1.06776000 & -1.01925900 \\ \mathrm{C} & 5.12855600 & -0.45927800 & 1.01348500 \\ \mathrm{H} & 3.39676400 & -0.00674900 & 2.20870700 \\ \mathrm{C} & 4.69342900 & -1.19480900 & -1.24455800 \\ \mathrm{H} & 2.62196900 & -1.31765400 & -1.81413600 \\ \mathrm{C} & 5.60017400 & -0.88922300 & -0.22801900 \\ \mathrm{H} & 5.82782400 & -0.22836400 & 1.81216200 \\ \mathrm{H} & 5.05280300 & -1.53848000 & -2.21048500 \\ \mathrm{H} & 6.66761000 & -0.99213100 & -0.40025400\end{array}$

\section{IM3}

Calculation Method $=$ RB3LYP

Basis Set $=6-31 \mathrm{G}(\mathrm{d})$

Charge $=1$

Spin $=$ Singlet

$\mathrm{E}(\mathrm{RB} 3 \mathrm{LYP})=-848.75037659$ a.u.

RMS Gradient Norm $=0.00000344$ a.u.

Imaginary Freq =

Dipole Moment $=3.2044$ Debye

\section{1}

$\mathrm{C}$

$\mathrm{H}$

$\mathrm{H}$

1.73977400

$-1.85417000$

0.38544800

$-2.20235700$

$-0.64918400$

1.82059100

2.66439900

1.64458400

$-2.14971500$

$-0.30775000$

0.06191400

0.88982700

0.38258800

$\mathrm{H}$

1.58015600

0.36220900

1.40958600

C

2.92785100

0.16530700

$-0.24590100$

0.49520500

$-0.41964300$

1.19263700

$-0.43446500$

C

$-0.40092400$

1.94590500

$-0.15443300$

C

$-1.38406400$

1.52207600

1.05714300

$-1.29167800$

2.98493100

$-1.15392600$

C

0.33075000

1.71915400

1.24058300

$\mathrm{H}$

$-2.27603900$

2.56322400

1.82334100

$\mathrm{H}$

$-1.40134700$

0.94972400

$-0.95680100$

C

$-2.22766600$

3.29361100

$-2.07595600$

$-1.26937300$

3.56852200

0.23693400

$-3.00248900$

2.81607100

2.15518600

$\mathrm{H}$

$-2.92261500$

4.11437900

$-1.72207600$

$\mathrm{H}$

2.65234500

1.74797000

0.39096300

2.96044000

$-0.07891300$

$-0.15175800$

$\mathrm{O}$

3.84420300

0.07425100

$-1.58773300$

4.22201000

0.02157600

$-1.95988900$

C

4.48188800

$-1.03499500$

0.49113500

$\mathrm{H}$

5.04626200

0.60518300

0.39686700

4.15365300

0.28401900

0.05776000

$\mathrm{H}$

3.45765900

2.24970400

1.55065500

H

0.45113600

$-0.25514000$

$-0.36141600$

$\mathrm{H}$

0.55285200

$-2.52522800$

$-1.43499300$

$\mathrm{H}$

0.57393500

$-2.26884000$

1.10109700

0.69868900

$-3.61226000$

2.16695000

1.04553800 


$\begin{array}{lrrr}\mathrm{C} & -0.79614400 & -2.15760600 & 0.52426800 \\ \mathrm{C} & -1.79445900 & -1.58684200 & 1.33319600 \\ \mathrm{C} & -1.09957300 & -2.41310600 & -0.83042900 \\ \mathrm{C} & -3.03553300 & -1.24636900 & 0.80431800 \\ \mathrm{H} & -1.58964100 & -1.41064900 & 2.38636600 \\ \mathrm{C} & -2.34543500 & -2.07968200 & -1.36150300 \\ \mathrm{H} & -0.36152200 & -2.90012000 & -1.46367600 \\ \mathrm{C} & -3.31288600 & -1.48486800 & -0.54844400 \\ \mathrm{H} & -3.79466000 & -0.80574800 & 1.44411700 \\ \mathrm{H} & -2.56592100 & -2.29814800 & -2.40252100 \\ \mathrm{H} & -4.28741900 & -1.23252400 & -0.95636400\end{array}$

\section{IM3,}

Calculation Method $=$ RB3LYP

Basis Set $=6-31 \mathrm{G}(\mathrm{d})$

Charge $=1$

Spin $=$ Singlet

$\mathrm{E}(\mathrm{RB} 3 \mathrm{LYP})=-848.74426356$ a.u.

RMS Gradient Norm $=0.00000305$ a.u.

Imaginary Freq =

Dipole Moment $=6.8061$ Debye

11

C

$\mathrm{H}$

0.82290200

0.84430800

0.01588500

1.02427100

1.06408900

$-1.03762700$

$\mathrm{H}$

1.41816700

1.54558700

0.61169600

$\mathrm{C}$

$-0.68093100$

1.14687300

0.32206400

0.96619200

2.64200300

1.38289500

$-0.87509100$

$-1.05616000$

0.33519300

0.00094600

C

$-1.55644500$

$-0.73510400$

$-0.54059100$

$\mathrm{C}$

$-2.39270600$

$-1.21385900$

$-0.20943300$

C

$-2.55564400$

$-1.37100700$

1.12807500

$-3.11976200$

$-2.27612100$

$-1.26520600$

$-3.39934800$

$-0.73865500$

1.38377400

$\mathrm{H}$

$-2.01970800$

$-2.43411900$

1.94165800

$-3.96101700$

$-1.00443300$

$-0.99690000$

$-3.00097600$

$-2.88436200$

$-2.28088400$

$-4.09871600$

$-2.64254300$

0.32527200

$-3.52794600$

$-2.91683400$

2.39684100

$-4.51210400$

$-3.71907200$

$-1.79702300$

$-4.76115000$

2.62353300

0.53730400

$-2.46511100$

2.93233400

0.11896700

$-0.75437600$

3.47397800

$-1.35066600$

0.04872900

3.64778400

$-1.40132300$

$-0.42161100$

3.66123900

0.95699000

C

0.67099900

4.65174600

0.88344900

$-0.79092500$

3.42141100

0.71750800

$\mathrm{H}$

$-0.69775100$

3.48099500

1.99015100

$\mathrm{H}$

$-2.80400600$

0.61963200

$-0.19426100$

$-1.52137100$

$-0.59322100$

$-1.59275200$

1.26253500

0.34928900

$\mathrm{H}$

0.99734900

$-0.82518600$

1.38903200 


$\begin{array}{lrrr}\mathrm{H} & 0.71281500 & -1.30389800 & -0.28313300 \\ \mathrm{C} & 2.75295900 & -0.76901700 & 0.14934200 \\ \mathrm{C} & 3.63816300 & -0.65898600 & 1.23021100 \\ \mathrm{C} & 3.27694600 & -1.00491500 & -1.12993800 \\ \mathrm{C} & 5.01481100 & -0.77954100 & 1.03844900 \\ \mathrm{H} & 3.24707300 & -0.48628000 & 2.23086100 \\ \mathrm{C} & 4.65215700 & -1.12705400 & -1.32470100 \\ \mathrm{H} & 2.60299500 & -1.10595000 & -1.97868700 \\ \mathrm{C} & 5.52491700 & -1.01255900 & -0.24003900 \\ \mathrm{H} & 5.68763400 & -0.69777200 & 1.88730200 \\ \mathrm{H} & 5.04253000 & -1.31780600 & -2.32032700 \\ \mathrm{H} & 6.59605700 & -1.11116400 & -0.39002200\end{array}$

\section{TS3}

Calculation Method $=$ RB3LYP

Basis Set $=6-31 \mathrm{G}(\mathrm{d})$

Charge $=1$

Spin $=$ Singlet

$\mathrm{E}(\mathrm{RB} 3 \mathrm{LYP})=-848.73743777$ a.u.

RMS Gradient Norm $=0.00000363$ a.u.

Imaginary Freq $=1$

Dipole Moment $=1.7713$ Debye

11

C $\quad-1.49856300$

$\mathrm{H} \quad-1.84610800$

2.06285600

$-0.49798200$

$2.22324400 \quad 0.52816500$

$\mathrm{H}$

$-1.45958700$

3.04140000

$-0.98301800$

C

$-0.08869900$

1.44750400

$-0.46286800$

$\mathrm{H}$

0.34046700

1.45591800

$-1.47151800$

C

$-0.02642300$

0.03657100

0.11109200

$\mathrm{H}$

$-0.45008500$

$-0.01904800$

1.11097500

C

1.18539700

$-0.77641000$

$-0.05026800$

C

1.64730300

$-1.52277900$

1.05154600

1.09490000

$-1.49206400$

1.98693400

1.90881100

$-0.83045500$

$-1.25878400$

$\mathrm{H}$

1.56096100

$-0.28231300$

$-2.13043600$

3.06754200

$-1.59147000$

$-1.35362200$

$\mathrm{H}$

3.61580000

$-1.62942200$

$-2.29001100$

3.52758800

$-2.30835800$

$-0.24274000$

$\mathrm{H}$

4.43497700

$-2.90016100$

$-0.31856000$

2.81756100

$-2.26937300$

0.96065400

3.17591600

$-2.82216800$

1.82357200

$-2.53200300$

1.19661400

$-1.26184600$

C

$-2.24346400$

1.12150600

$-2.31726000$

$\mathrm{H}$

$-3.51133400$

1.68276500

$-1.21783900$

$-2.57059500$

$-0.15302500$

$-0.62927000$

C

$-1.45256300$

$-1.02320300$

$-0.85269400$

$\mathrm{H}$

$-0.93527500$

$-0.92692200$

$-1.80461600$

C

$-1.44898800$

$-2.32099500$

$-0.23879100$

$\mathrm{H}$

$-0.63775100$

$-3.00578600$

$-0.46427800$

C

$-3.54726100$

$-0.52376400$

0.29567300 


$\begin{array}{lrrr}\mathrm{H} & -4.37045100 & 0.15113500 & 0.51118100 \\ \mathrm{C} & -3.48462100 & -1.76823100 & 0.92187500 \\ \mathrm{H} & -4.26118000 & -2.04852300 & 1.62833500 \\ \mathrm{C} & -2.43649900 & -2.67462900 & 0.65165200 \\ \mathrm{H} & -2.42481200 & -3.64650500 & 1.13431900 \\ \mathrm{C} & 0.87096700 & 2.35674100 & 0.42803900 \\ \mathrm{C} & 0.87620500 & 3.82260600 & -0.02614200 \\ \mathrm{H} & -0.08553600 & 4.30503000 & 0.15434700 \\ \mathrm{H} & 1.63474600 & 4.36152700 & 0.55320600 \\ \mathrm{H} & 1.12352500 & 3.91913900 & -1.09021400 \\ \mathrm{O} & 0.38236400 & 2.21921800 & 1.73987700 \\ \mathrm{H} & 1.03872100 & 2.61761200 & 2.33632600 \\ \mathrm{O} & 2.19108600 & 1.85279300 & 0.41785800 \\ \mathrm{H} & 2.72701500 & 2.35634800 & -0.21463700\end{array}$

\section{IM4}

Calculation Method $=$ RB3LYP

Basis Set $=6-31 \mathrm{G}(\mathrm{d})$

Charge $=1$

Spin $=$ Singlet

$\mathrm{E}(\mathrm{RB} 3 \mathrm{LYP})=-848.73970475$ a.u.

RMS Gradient Norm $=0.00000534$ a.u.

Imaginary Freq =

Dipole Moment $=5.0366$ Debye

11

C

$\mathrm{H}$

$\mathrm{H}$

$\mathrm{C}$

$\mathrm{H}$

C

$\mathrm{C}$

$\mathrm{C}$

$\mathrm{C}$

C

$\mathrm{C}$

$\mathrm{H}$

$\mathrm{C}$

$\mathrm{H}$

C

$\mathrm{H}$

$\mathrm{H}$

$\mathrm{H}$

O

$\mathrm{O}$

$\mathrm{H}$

C

$\mathrm{H}$

$\mathrm{H}$

$\mathrm{H}$
1.46921200

1.78621600

1.43023700

0.07972900

$-0.30950600$

$-0.92235300$

0.09850800

$-1.17054000$

$-1.74111300$

$-1.78512600$

$-2.90948800$

$-1.28498900$

$-2.95644600$

$-1.35644700$

$-3.52083500$

$-3.34371800$

$-3.42844300$

$-4.42987100$

$-2.19906000$

$-0.51607600$

$-0.99730500$

$-0.92230600$

0.01495100

$-1.72596400$

$-1.11899500$
$-2.10020200$

$-2.28229000$

$-3.06954000$

$-1.44385000$

$-1.41681600$

$-2.32190900$

0.00511400

0.80776900

1.05385500

1.34767700

1.80381100

0.64832600

2.09560000

1.15916000

2.32684000

1.97973500

2.49613600

2.91412000

$-1.77257600$

$-2.17743600$

$-2.82066300$

$-3.80091200$

$-4.30186000$

$-4.32057800$

$-3.91595800$
$-0.46266300$

0.57010800

$-0.96592700$

$-0.46186100$

$-1.48645800$

0.36301200

0.10892700

$-0.04592400$

$-1.30360500$

1.09267200

$-1.41864300$

$-2.20487600$

0.97998100

2.07383900

$-0.27608300$

$-2.39838000$

1.87239900

$-0.36605400$

0.13173100

1.71766400

2.26298200

$-0.04423200$

0.20788700

0.49561400

$-1.11453300$ 


$\begin{array}{lrrr}\mathrm{H} & -2.87441300 & -2.38990300 & 0.45716300 \\ \mathrm{H} & 0.37109500 & -0.04949600 & 1.16444800 \\ \mathrm{C} & 2.54122900 & -1.24824200 & -1.19120200 \\ \mathrm{H} & 2.26445500 & -1.15121700 & -2.24937900 \\ \mathrm{H} & 3.51564800 & -1.74253400 & -1.14320800 \\ \mathrm{C} & 2.58029000 & 0.09049600 & -0.54966200 \\ \mathrm{C} & 1.34560400 & 0.88457200 & -0.61559300 \\ \mathrm{C} & 3.66209700 & 0.54330000 & 0.19127100 \\ \mathrm{C} & 1.39687700 & 2.22100500 & -0.00854400 \\ \mathrm{H} & 0.97642700 & 0.94426600 & -1.64775300 \\ \mathrm{C} & 3.62109200 & 1.80583300 & 0.79109900 \\ \mathrm{H} & 4.55316700 & -0.07005100 & 0.28497100 \\ \mathrm{C} & 2.49520200 & 2.65629600 & 0.68126200 \\ \mathrm{H} & 0.51056800 & 2.84190600 & -0.08917900 \\ \mathrm{H} & 4.48522700 & 2.14766300 & 1.35573700 \\ \mathrm{H} & 2.51293100 & 3.63736700 & 1.14416200\end{array}$

\section{TS4}

Calculation Method $=$ RB3LYP

Basis Set $=6-31 \mathrm{G}(\mathrm{d})$

Charge $=1$

Spin $=$ Singlet

$\mathrm{E}(\mathrm{RB} 3 \mathrm{LYP})=-848.73240128$ a.u.

RMS Gradient Norm $=0.00000528$ a.u.

Imaginary Freq $=1$

Dipole Moment $=6.2177$ Debye

11

C $\quad 0.48223200$

$\mathrm{H} \quad 0.71883200$

$-2.36221100$

$-0.43387000$

$-2.55901800 \quad 0.61967300$

$\mathrm{H}$

0.07901000

$-3.28765100$

$-0.86282600$

C

$-0.53795700$

$-1.24105200$

$-0.52452100$

$\mathrm{H} \quad-0.98173700$

$-1.10586400$

$-1.51201200$

C

$-2.25177700$

$-1.94390000$

0.44577300

C $\quad-0.33252800$

$-0.08639800$

0.23253500

C $\quad-0.93340300$

1.20177600

0.05156800

C -1.64435800

1.56871200

$-1.12009900$

C $\quad-0.82665500$

2.14400800

1.10343600

C -2.23999000

2.81682700

0.88652400

$-1.21730200$

$\mathrm{H}$

$-1.69478300$

3.39304000

$-1.96400100$

$-1.42569300$

1.87516500

1.00117000

$\mathrm{H}$

$-0.27575700$

3.72888300

2.00030200

C $\quad-2.13512500$

$-2.77441400$

3.09632100

$-0.15588900$

$\mathrm{H}$

$-1.34227700$

4.10528500

$-2.11999900$

$\mathrm{H}$

$-2.59990600$

4.70687000

1.81578100

$\mathrm{O}$

$-3.01543200$

$-0.89058800$

$-0.23989100$

$\mathrm{O}$

$-1.79368500$

$-2.48824700$

0.76185800

$\mathrm{H}$

$-1.38382100$

$-3.35067500$

1.58118500

$\mathrm{C}$

$-2.78677300$

$-2.87601000$

1.40318900

$-2.06565000$

$-3.64806200$

$-0.62407500$

$-0.89784200$ 


$\begin{array}{lrrr}\mathrm{H} & -3.68317000 & -3.36000400 & -0.21567100 \\ \mathrm{H} & -3.06602300 & -2.32991300 & -1.52868700 \\ \mathrm{H} & -3.47161600 & -0.54402400 & -0.02239800 \\ \mathrm{H} & 0.25081200 & -0.20337700 & 1.14485700 \\ \mathrm{C} & 1.80496300 & -2.02376100 & -1.17906300 \\ \mathrm{H} & 1.58305800 & -1.82469800 & -2.23401000 \\ \mathrm{H} & 2.43213800 & -2.92333100 & -1.15622200 \\ \mathrm{C} & 2.54934000 & -0.85568300 & -0.57194400 \\ \mathrm{C} & 2.54553300 & 0.40585000 & -1.18601200 \\ \mathrm{C} & 3.24670300 & -1.00873600 & 0.63796500 \\ \mathrm{C} & 3.21926500 & 1.48611100 & -0.60989600 \\ \mathrm{H} & 2.03030000 & 0.53725600 & -2.13494200 \\ \mathrm{C} & 3.91683500 & 0.06739900 & 1.21680000 \\ \mathrm{H} & 3.28098900 & -1.98410500 & 1.11941800 \\ \mathrm{C} & 3.90317700 & 1.31998600 & 0.59389600 \\ \mathrm{H} & 3.21725400 & 2.45151300 & -1.10802200 \\ \mathrm{H} & 4.46211400 & -0.07197500 & 2.14597800 \\ \mathrm{H} & 4.43485700 & 2.15539900 & 1.04014500\end{array}$

\section{TS4'}

Calculation Method $=$ RB3LYP

Basis Set $=6-31 \mathrm{G}(\mathrm{d})$

Charge $=1$

Spin $=$ Singlet

$\mathrm{E}(\mathrm{RB} 3 \mathrm{LYP})=-848.73586636$ a.u.

RMS Gradient Norm $=0.00000275$ a.u. Imaginary Freq $=1$

Dipole Moment $=7.1721$ Debye

11

$\mathrm{C}$

$\mathrm{H}$

$-1.04637800$

0.49353000

0.01029300

$0.45676300 \quad 1.09651600$
1.39743600

$-1.20512600$

1.39743600

$-0.35867000$

$\mathrm{H}$

$-1.54066000$

0.52920100

$-0.29039600$

$\mathrm{H} \quad 0.70245700$

0.70095800

$-1.33662800$

1.17425200

2.26172500

0.38291100

C

1.26178000

$-0.38181000$

0.40018000

$-0.83445100$

0.07724600

$\mathrm{C}$

2.57253600

$-0.52802400$

$-1.15082300$

3.26135000

$-1.63278700$

1.03268000

$\begin{array}{ll}\mathrm{C} & 4.50380000\end{array}$

$-0.99174800$

$-1.39680800$

2.71106800

0.06101500

$-1.90396400$

C

4.54204400

$-2.09575800$

0.77681400

2.76959900

$-1.87887400$

1.97011200

5.16362300

$-1.77429400$

$-0.43723000$

C

4.99513200

$-0.75828800$

$-2.33584900$

$\mathrm{H}$

5.05996500

$-2.70557500$

1.50991600

$\mathrm{H}$

6.16553700

$-2.14067700$

$-0.64139200$

2.49807500

2.23551800

0.16153900

$\mathrm{O}$

0.88455000

2.31030000

1.70740400

$\mathrm{H}$

$-0.04741700$

2.54725300

1.85063500 


$\begin{array}{lrrr}\mathrm{C} & 0.49382900 & 3.26698200 & -0.52272700 \\ \mathrm{H} & -0.59195400 & 3.24611900 & -0.42469200 \\ \mathrm{H} & 0.85850400 & 4.25710100 & -0.22487400 \\ \mathrm{H} & 0.76904000 & 3.09808900 & -1.56485500 \\ \mathrm{H} & 2.96024700 & 1.86832600 & 0.93781900 \\ \mathrm{H} & 0.88224400 & -0.72176200 & 1.36568900 \\ \mathrm{C} & -1.75034000 & -0.72875200 & -0.63564500 \\ \mathrm{H} & -1.57037300 & -0.71153600 & -1.71779300 \\ \mathrm{H} & -1.28566900 & -1.64720500 & -0.25370300 \\ \mathrm{C} & -3.23693300 & -0.73419400 & -0.35356900 \\ \mathrm{C} & -4.13399100 & -0.11334900 & -1.23302400 \\ \mathrm{C} & -3.73941300 & -1.32725400 & 0.81279200 \\ \mathrm{C} & -5.50064200 & -0.08349800 & -0.95358600 \\ \mathrm{H} & -3.76245100 & 0.33940900 & -2.15029300 \\ \mathrm{C} & -5.10536200 & -1.30066300 & 1.09463300 \\ \mathrm{H} & -3.05867500 & -1.82699600 & 1.49969200 \\ \mathrm{C} & -5.98923100 & -0.67637600 & 0.21210600 \\ \mathrm{H} & -6.18411000 & 0.39423100 & -1.64982200 \\ \mathrm{H} & -5.48026900 & -1.77374900 & 1.99785900 \\ \mathrm{H} & -7.05359600 & -0.65945400 & 0.42766800\end{array}$

\section{cis-TS3}

Calculation Method $=$ RB3LYP

Basis Set $=6-31 \mathrm{G}(\mathrm{d})$

Charge $=1$

Spin $=$ Singlet

$\mathrm{E}(\mathrm{RB} 3 \mathrm{LYP})=-848.72997920$ a.u.

RMS Gradient Norm $=0.00000096$ a.u.

Imaginary Freq $=1$

Dipole Moment $=2.9101$ Debye

$\begin{array}{crrc}11 & & & \\ \mathrm{C} & -0.74740900 & 1.78978300 & 1.26524800 \\ \mathrm{H} & -0.15701900 & 1.28914500 & 2.03670200 \\ \mathrm{H} & -0.63695200 & 2.85981600 & 1.45436100 \\ \mathrm{C} & -0.22415200 & 1.42920500 & -0.14054000 \\ \mathrm{H} & -0.99064800 & 1.75164100 & -0.85571000 \\ \mathrm{C} & 1.05239200 & 2.22352200 & -0.55378000 \\ \mathrm{C} & -0.07281700 & -0.08684300 & -0.39733500 \\ \mathrm{C} & -2.24294700 & 1.41153900 & 1.36854000 \\ \mathrm{H} & -2.87379500 & 2.21108200 & 0.96442400 \\ \mathrm{H} & -2.52289000 & 1.30223900 & 2.42672100 \\ \mathrm{C} & -2.53248900 & 0.12981100 & 0.64396300\end{array}$




$\begin{array}{lrrr}\mathrm{C} & -3.67955200 & -0.06908100 & -0.11879400 \\ \mathrm{C} & -1.51962400 & -0.89350800 & 0.66530600 \\ \mathrm{C} & -3.88616700 & -1.28645200 & -0.77369800 \\ \mathrm{C} & -1.79343700 & -2.15550200 & 0.03117000 \\ \mathrm{C} & -2.94703700 & -2.33629200 & -0.69733500 \\ \mathrm{H} & -4.79203700 & -1.42647800 & -1.35747200 \\ \mathrm{H} & -1.05341200 & -2.94668400 & 0.10514300 \\ \mathrm{H} & -3.14368000 & -3.27588400 & -1.20325400 \\ \mathrm{H} & -4.42552700 & 0.71705900 & -0.19155300 \\ \mathrm{H} & -0.90143600 & -0.92716000 & 1.55897100 \\ \mathrm{C} & 1.11873500 & -0.88687700 & -0.05966300 \\ \mathrm{H} & -0.43847600 & -0.33868900 & -1.38937300 \\ \mathrm{C} & 1.52131500 & -1.87281400 & -0.98487800 \\ \mathrm{H} & 0.95749500 & -2.00277000 & -1.90544600 \\ \mathrm{C} & 2.64399600 & -2.65910600 & -0.74978300 \\ \mathrm{H} & 2.95030000 & -3.39977200 & -1.48208600 \\ \mathrm{C} & 3.37174200 & -2.49319300 & 0.43182600 \\ \mathrm{H} & 4.24250500 & -3.11278500 & 0.62514900 \\ \mathrm{C} & 2.97610600 & -1.53192800 & 1.36804500 \\ \mathrm{H} & 3.54165100 & -1.40294900 & 2.28582900 \\ \mathrm{C} & 1.86253000 & -0.73482700 & 1.12817500 \\ \mathrm{H} & 1.58547700 & 0.01797000 & 1.85523300 \\ \mathrm{C} & 0.73140200 & 3.68718100 & -0.88112300 \\ \mathrm{H} & 0.31926100 & 4.21721400 & -0.01897400 \\ \mathrm{H} & 1.65658200 & 4.20585800 & -1.16712000 \\ \mathrm{H} & 0.03077200 & 3.76118400 & -1.71820500 \\ \mathrm{O} & 1.54736100 & 1.56901900 & -1.70886700 \\ \mathrm{H} & 2.11577200 & 2.17909700 & -2.20636900 \\ \mathrm{O} & 1.94968400 & 2.14474900 & 0.53427200 \\ \mathrm{H} & 2.76416400 & 2.62300000 & 0.30772500\end{array}$

\section{cis-IM4}

Calculation Method $=$ RB3LYP

Basis Set $=6-31 \mathrm{G}(\mathrm{d})$

Charge $=1$

Spin $=$ Singlet

$\mathrm{E}(\mathrm{RB} 3 \mathrm{LYP})=-848.73156176$ a.u.

RMS Gradient Norm $=0.00000941$ a.u.

Imaginary Freq =

Dipole Moment $=5.6811$ Debye

11

$\mathrm{C}$

$\mathrm{H}$

$\mathrm{H}$

C

$\mathrm{H}$

$\mathrm{C}$

C

$\mathrm{O}$

$\mathrm{O}$
$-0.74313000$

$-0.20404900$

$-0.61572900$

$-0.16693600$

$-0.86238500$

1.18660200

$-0.15177600$

1.63101500

2.03770800
1.84598400

1.32790300

2.91257700

1.46476600

1.85403900

2.14832400

$-0.08410800$

1.53089700

1.89261900
1.21297100

2.01136100

1.41151200

$-0.16093400$

$-0.91714300$

$-0.48260400$

$-0.40070400$

$-1.67957400$

0.61479200 


$\begin{array}{lrrr}\mathrm{H} & 2.86637500 & 2.38529600 & 0.49818200 \\ \mathrm{C} & 1.04305500 & 3.65933200 & -0.71051200 \\ \mathrm{H} & 0.69090400 & 4.17895100 & 0.18431300 \\ \mathrm{H} & 2.02465300 & 4.08220500 & -0.96526100 \\ \mathrm{H} & 0.36444300 & 3.87118100 & -1.54195200 \\ \mathrm{H} & 2.36708500 & 2.04486900 & -2.04934000 \\ \mathrm{C} & -2.25017400 & 1.51779200 & 1.23832600 \\ \mathrm{H} & -2.84251600 & 2.33296700 & 0.80475700 \\ \mathrm{H} & -2.60103200 & 1.41790900 & 2.27799700 \\ \mathrm{C} & -2.56031900 & 0.24436100 & 0.51595800 \\ \mathrm{C} & -3.79085200 & -0.02240100 & -0.06383900 \\ \mathrm{C} & -1.47229300 & -0.73719400 & 0.41229300 \\ \mathrm{C} & -4.02223300 & -1.26254300 & -0.67075700 \\ \mathrm{C} & -1.80001700 & -2.03063900 & -0.19326300 \\ \mathrm{C} & -3.03554700 & -2.27520400 & -0.72989900 \\ \mathrm{H} & -4.99714800 & -1.45479300 & -1.11239800 \\ \mathrm{H} & -1.00817900 & -2.77269700 & -0.24495800 \\ \mathrm{H} & -3.26464900 & -3.22407700 & -1.20338400 \\ \mathrm{H} & -4.58507900 & 0.71777800 & -0.03118600 \\ \mathrm{H} & -1.02666100 & -0.87943400 & 1.40647700 \\ \mathrm{C} & 1.05985100 & -0.91995600 & -0.04857500 \\ \mathrm{C} & 1.55049500 & -1.05369800 & 1.25962000 \\ \mathrm{C} & 1.70003900 & -1.62775300 & -1.07711500 \\ \mathrm{C} & 2.65628600 & -1.85646000 & 1.52736600 \\ \mathrm{H} & 1.08574200 & -0.51664700 & 2.08236100 \\ \mathrm{C} & 2.80988500 & -2.43032900 & -0.81297900 \\ \mathrm{H} & 1.33959400 & -1.53024400 & -2.09794200 \\ \mathrm{C} & 3.28990400 & -2.54882100 & 0.49139400 \\ \mathrm{H} & 3.02516100 & -1.94164000 & 2.54523700 \\ \mathrm{H} & 3.29505700 & -2.96306300 & -1.62557100 \\ \mathrm{H} & 4.14830300 & -3.17952400 & 0.70303200 \\ \mathrm{H} & -0.36671200 & -0.24040800 & -1.45832100\end{array}$

\section{cis-IM3}

Calculation Method $=$ RB3LYP

Basis Set $=6-31 \mathrm{G}(\mathrm{d})$

Charge $=1$

Spin $=$ Singlet

$\mathrm{E}(\mathrm{RB} 3 \mathrm{LYP})=-848.74420318$ a.u.

RMS Gradient Norm $=0.00000871$ a.u.

Imaginary Freq =

Dipole Moment $=3.2540$ Debye

11

$\begin{array}{rrrr}\mathrm{C} & 1.29838000 & 1.41821200 & 1.15603900 \\ \mathrm{H} & 0.97411800 & 0.65397400 & 1.86608600 \\ \mathrm{H} & 2.23322800 & 1.83009000 & 1.54883900 \\ \mathrm{C} & 1.57846400 & 0.79054800 & -0.23261400 \\ \mathrm{H} & 1.72914000 & 1.61286200 & -0.93965900 \\ \mathrm{C} & 2.91455900 & -0.02893600 & -0.30068400\end{array}$




\begin{tabular}{lrrr}
$\mathrm{C}$ & 0.44904000 & -0.02327200 & -0.80525300 \\
$\mathrm{O}$ & 2.81174800 & -0.72134700 & -1.53541600 \\
$\mathrm{O}$ & 2.91853100 & -0.91166700 & 0.80053700 \\
$\mathrm{H}$ & 3.81301200 & -1.26832800 & 0.92657200 \\
$\mathrm{C}$ & 4.15331900 & 0.86749900 & -0.28770000 \\
$\mathrm{H}$ & 4.23761600 & 1.43516400 & 0.64247600 \\
$\mathrm{H}$ & 5.05380300 & 0.24383900 & -0.37640200 \\
$\mathrm{H}$ & 4.14442500 & 1.56020000 & -1.13338300 \\
$\mathrm{H}$ & 3.66915000 & -1.12061100 & -1.75906900 \\
$\mathrm{C}$ & 0.24596000 & 2.54467700 & 1.07035200 \\
$\mathrm{H}$ & 0.64251700 & 3.36419900 & 0.45905700 \\
$\mathrm{H}$ & 0.09718000 & 2.94913600 & 2.07865100 \\
$\mathrm{C}$ & -1.07279200 & 2.07213800 & 0.50417200 \\
$\mathrm{C}$ & -1.40126200 & 2.29669700 & -0.84759000 \\
$\mathrm{C}$ & -1.99494700 & 1.37979500 & 1.30789600 \\
$\mathrm{C}$ & -2.60594300 & 1.83027300 & -1.38484100 \\
$\mathrm{C}$ & -3.18856400 & 0.90627600 & 0.77397400 \\
$\mathrm{C}$ & -3.49660300 & 1.12582200 & -0.57818200 \\
$\mathrm{H}$ & -2.85100700 & 2.03614900 & -2.42297700 \\
$\mathrm{H}$ & -3.89454100 & 0.38008300 & 1.41017200 \\
$\mathrm{H}$ & -4.43974400 & 0.77178700 & -0.98418700 \\
$\mathrm{H}$ & -0.72549500 & 2.87942400 & -1.47012800 \\
$\mathrm{C}$ & -0.22919300 & -1.15334100 & -0.32000800 \\
$\mathrm{C}$ & 0.05561200 & -1.80928500 & 0.91815200 \\
$\mathrm{C}$ & -1.26019100 & -1.68973800 & -1.15521700 \\
$\mathrm{C}$ & -0.64867000 & -2.94204700 & 1.27738100 \\
$\mathrm{H}$ & 0.85999500 & -1.44569900 & 1.54209200 \\
$\mathrm{C}$ & -1.96283900 & -2.82087000 & -0.77888900 \\
$\mathrm{H}$ & -1.48242400 & -1.19734600 & -2.09671300 \\
$\mathrm{C}$ & -1.65580100 & -3.44657300 & 0.43605300 \\
$\mathrm{H}$ & -0.42133100 & -3.44961100 & 2.20928200 \\
$\mathrm{H}$ & -2.73933600 & -3.22407000 & -1.42054200 \\
$\mathrm{H}$ & -2.20125100 & -4.33846100 & 0.73197100 \\
$\mathrm{H}$ & 0.16082400 & 0.26402700 & -1.81185400 \\
$\mathrm{H}$ & -1.77328100 & 1.22005200 & 2.36051000 \\
& & & \\
\hline
\end{tabular}

\section{cis-IM3,}

Calculation Method $=$ RB3LYP

Basis Set $=6-31 \mathrm{G}(\mathrm{d})$

Charge $=1$

Spin $=$ Singlet

$\mathrm{E}(\mathrm{RB} 3 \mathrm{LYP})=-848.73769943$ a.u.

RMS Gradient Norm $=0.00000177$ a.u.

Imaginary Freq =

Dipole Moment $=6.4775$ Debye

11

$\begin{array}{lrrr}\mathrm{C} & -0.62534900 & 0.92066000 & -0.07488700 \\ \mathrm{H} & -0.46358700 & 0.29007900 & 0.80350800 \\ \mathrm{H} & -1.18929400 & 1.79654900 & 0.26611100 \\ \mathrm{C} & 0.72712300 & 1.42122400 & -0.64733100\end{array}$ 


$\begin{array}{lrrr}\mathrm{H} & 0.50643900 & 2.04192000 & -1.52195900 \\ \mathrm{C} & 1.50960300 & 2.37769500 & 0.34860300 \\ \mathrm{C} & 1.64760100 & 0.35252500 & -1.13130100 \\ \mathrm{O} & 2.81314900 & 2.39767100 & -0.18508600 \\ \mathrm{O} & 1.58897000 & 1.81967200 & 1.64576600 \\ \mathrm{H} & 0.91865100 & 2.22992700 & 2.21570300 \\ \mathrm{C} & 0.89281700 & 3.77657500 & 0.38952700 \\ \mathrm{H} & -0.14135400 & 3.76610900 & 0.75006100 \\ \mathrm{H} & 1.48292700 & 4.40664400 & 1.06532100 \\ \mathrm{H} & 0.90986700 & 4.23068600 & -0.60402400 \\ \mathrm{H} & 3.38084300 & 2.91682900 & 0.41134300 \\ \mathrm{C} & -1.47574400 & 0.16123400 & -1.11347700 \\ \mathrm{H} & -1.62910300 & 0.80145100 & -1.99147700 \\ \mathrm{H} & -0.92075500 & -0.72174000 & -1.46163000 \\ \mathrm{C} & -2.81178500 & -0.26156900 & -0.54331600 \\ \mathrm{C} & -3.95449500 & 0.52637500 & -0.73692700 \\ \mathrm{C} & -2.92323700 & -1.43300200 & 0.21953100 \\ \mathrm{C} & -5.17858200 & 0.15511100 & -0.18013100 \\ \mathrm{H} & -3.88779500 & 1.43178900 & -1.33657900 \\ \mathrm{C} & -4.14545300 & -1.80844600 & 0.77572400 \\ \mathrm{H} & -2.04858000 & -2.06421500 & 0.36740400 \\ \mathrm{C} & -5.27668100 & -1.01319200 & 0.57805900 \\ \mathrm{H} & -6.05650800 & 0.77319500 & -0.34483300 \\ \mathrm{H} & -4.21807800 & -2.72384700 & 1.35621700 \\ \mathrm{H} & -6.23055500 & -1.30644500 & 1.00664600 \\ \mathrm{C} & 2.15766500 & -0.79039500 & -0.50839800 \\ \mathrm{C} & 1.94778000 & -1.12832400 & 0.86813300 \\ \mathrm{C} & 2.94501700 & -1.67244900 & -1.32133500 \\ \mathrm{C} & 2.49203400 & -2.28862000 & 1.38256200 \\ \mathrm{H} & 1.41018700 & -0.43991300 & 1.50590400 \\ \mathrm{C} & 3.46793700 & -2.83752800 & -0.79701800 \\ \mathrm{H} & 3.11797500 & -1.41453900 & -2.36234700 \\ \mathrm{C} & 3.23898400 & -3.14461700 & 0.55396400 \\ \mathrm{H} & 2.34644200 & -2.54214400 & 2.42746100 \\ \mathrm{H} & 4.05499100 & -3.50687600 & -1.41686800 \\ \mathrm{H} & 3.65295700 & -4.05935400 & 0.96943700 \\ \mathrm{H} & 1.97596300 & 0.47561300 & -2.16273700\end{array}$

\section{cis-TS2}

Calculation Method $=$ RB3LYP

Basis Set $=6-31 \mathrm{G}(\mathrm{d})$

Charge $=1$

Spin $=$ Singlet

$\mathrm{E}(\mathrm{RB} 3 \mathrm{LYP})=-848.73332119$ a.u.

RMS Gradient Norm $=0.00000445$ a.u.

Imaginary Freq $=1$

Dipole Moment $=8.2933$ Debye

11

$\begin{array}{lrrr}\mathrm{C} & 0.57816200 & -1.04159100 & -0.02672000 \\ \mathrm{H} & 0.47251900 & -0.42076200 & 0.86547700\end{array}$ 


$\begin{array}{lrrr}\mathrm{H} & 1.11355500 & -1.94414000 & 0.29354400 \\ \mathrm{C} & -0.78846200 & -1.48638700 & -0.56551300 \\ \mathrm{H} & -0.60971800 & -2.09575500 & -1.45883600 \\ \mathrm{C} & -1.69176000 & -2.32490400 & 0.36505400 \\ \mathrm{C} & -1.81380900 & -0.43247100 & -0.99214800 \\ \mathrm{O} & -2.99467700 & -1.86805800 & -0.23266500 \\ \mathrm{O} & -1.62516400 & -1.85715900 & 1.67033200 \\ \mathrm{H} & -1.79609400 & -2.58100800 & 2.29584700 \\ \mathrm{C} & -1.59212700 & -3.83075900 & 0.25553200 \\ \mathrm{H} & -0.59974100 & -4.16526300 & 0.57613600 \\ \mathrm{H} & -2.34922900 & -4.31008800 & 0.88785900 \\ \mathrm{H} & -1.75841500 & -4.15666800 & -0.77355000 \\ \mathrm{H} & -3.55949800 & -1.54595400 & 0.49696300 \\ \mathrm{C} & 1.42919700 & -0.30335800 & -1.08202800 \\ \mathrm{H} & 1.54591300 & -0.94754700 & -1.96367200 \\ \mathrm{H} & 0.89512500 & 0.59569900 & -1.41836100 \\ \mathrm{C} & 2.79065600 & 0.08534500 & -0.54384700 \\ \mathrm{C} & 3.87429300 & -0.79735900 & -0.63972600 \\ \mathrm{C} & 2.98265600 & 1.31922300 & 0.09100800 \\ \mathrm{C} & 5.11962100 & -0.45740200 & -0.11021700 \\ \mathrm{H} & 3.74651100 & -1.75407400 & -1.14249700 \\ \mathrm{C} & 4.22662200 & 1.66321600 & 0.62111600 \\ \mathrm{H} & 2.15453200 & 2.02224700 & 0.16170800 \\ \mathrm{C} & 5.29816900 & 0.77389800 & 0.52303500 \\ \mathrm{H} & 5.95158700 & -1.15024500 & -0.19931500 \\ \mathrm{H} & 4.36133000 & 2.62747300 & 1.10326100 \\ \mathrm{H} & 6.26864700 & 1.04198400 & 0.93042500 \\ \mathrm{C} & -2.10561700 & 0.84396000 & -0.42348700 \\ \mathrm{C} & -1.73748100 & 1.24006000 & 0.89197500 \\ \mathrm{C} & -2.79506200 & 1.77127400 & -1.25274700 \\ \mathrm{C} & -2.03208200 & 2.51857000 & 1.33624600 \\ \mathrm{H} & -1.26222600 & 0.53034200 & 1.55692400 \\ \mathrm{C} & -3.07061200 & 3.05359500 & -0.80345500 \\ \mathrm{H} & -3.09020600 & 1.47227800 & -2.25489000 \\ \mathrm{C} & -2.68813300 & 3.42627300 & 0.49043700 \\ \mathrm{H} & -1.75581900 & 2.81932600 & 2.34175500 \\ \mathrm{H} & -3.58279100 & 3.76049300 & -1.44785500 \\ \mathrm{H} & -2.90730400 & 4.42850900 & 0.84743300 \\ \mathrm{H} & -2.18534300 & -0.55220900 & -2.00676900 \\ & & & \\ & & & \\ & & \end{array}$

\section{cis-IM2}

Calculation Method $=$ RB3LYP

Basis Set $=6-31 \mathrm{G}(\mathrm{d})$

Charge $=1$

Spin $=$ Singlet

$\mathrm{E}(\mathrm{RB} 3 \mathrm{LYP})=-848.73916701$ a.u. 
RMS Gradient Norm $=0.00000729$ a.u.

Imaginary Freq =

Dipole Moment $=10.1280$ Debye

11

$\mathrm{C}$

$-0.29622400$

$1.17089100 \quad-0.00646000$

$\mathrm{H}$

$\begin{array}{lll}-0.24198300 & 0.48612800 & 0.84377900\end{array}$

$\mathrm{H}$

$-0.72175600$

2.10772200

0.37424400

C

1.10358100

1.46236200

$-0.56160600$

$\mathrm{H}$

0.99806400

$2.08851800-1.45472200$

$\mathrm{C}$

2.07375200

2.18620700

0.38325700

$\mathrm{C}$

2.06782000

0.28705200

$-0.90060000$

$\mathrm{O}$

3.26231900

$0.94593400 \quad-0.22183300$

$\mathrm{O}$

1.86609600

$1.95705000 \quad 1.66985400$

$\mathrm{H}$

2.40119900

2.54481500

2.23714700

C

2.71325200

3.48403500

0.00088300

$\mathrm{H}$

1.95747900

4.27389100

0.10813100

$\mathrm{H}$

3.56378100

3.72272000

0.64910500

$\mathrm{H}$

3.05114000

3.46869400

$-1.03609300$

$\mathrm{H}$

3.61490100

0.34238000

0.46565700

C

$-1.24270300$

0.5927600

$-1.08078400$

$\mathrm{H}$

$-1.30553100$

1.29733500

$-1.92080200$

$\mathrm{H}$

$-0.81793400$

$-0.33769900$

$-1.47763000$

C

$-2.62681600$

0.32677200

$-0.52515800$

C

$-3.61466000$

1.31910100

$-0.56296100$

C

$-2.93183200$

$-0.90487800$

0.06878000

C

$-4.87815800$

1.08825200

$-0.01761900$

$\mathrm{H}$

$-3.39852500$

2.27669900

$-1.03292200$

$\mathrm{C}$

$-4.19420200$

$-1.13919800$

0.61487300

$\mathrm{H}$

$-2.17833100$

$-1.68975800$

0.09588100

C

$-5.17031000$

$-0.14187400$

0.57405500

$\mathrm{H}$

$-5.63582100$

1.86554300

$-0.06196700$

$\mathrm{H}$

$-4.41813000$

$-2.10236900$

1.06495500

$\mathrm{H}$

$-6.15520300$

$-0.32498000$

0.99379600

C

1.84308200

$-1.10254700$

$-0.39061500$

C

1.69326700

$-1.38773900$

0.97933400

C

1.81813200

$-2.15728800$

$-1.31584300$ 


$\begin{array}{lrrr}\mathrm{C} & 1.51909400 & -2.70329500 & 1.40589300 \\ \mathrm{H} & 1.70286600 & -0.58924000 & 1.71668900 \\ \mathrm{C} & 1.62909500 & -3.47014200 & -0.88626100 \\ \mathrm{H} & 1.94780500 & -1.95012800 & -2.37514900 \\ \mathrm{C} & 1.48199300 & -3.74442900 & 0.47456300 \\ \mathrm{H} & 1.40593000 & -2.91507000 & 2.46472400 \\ \mathrm{H} & 1.60824600 & -4.27701200 & -1.61217800 \\ \mathrm{H} & 1.34243400 & -4.76749100 & 0.81043900 \\ \mathrm{H} & 2.32307600 & 0.25918700 & -1.95965200\end{array}$



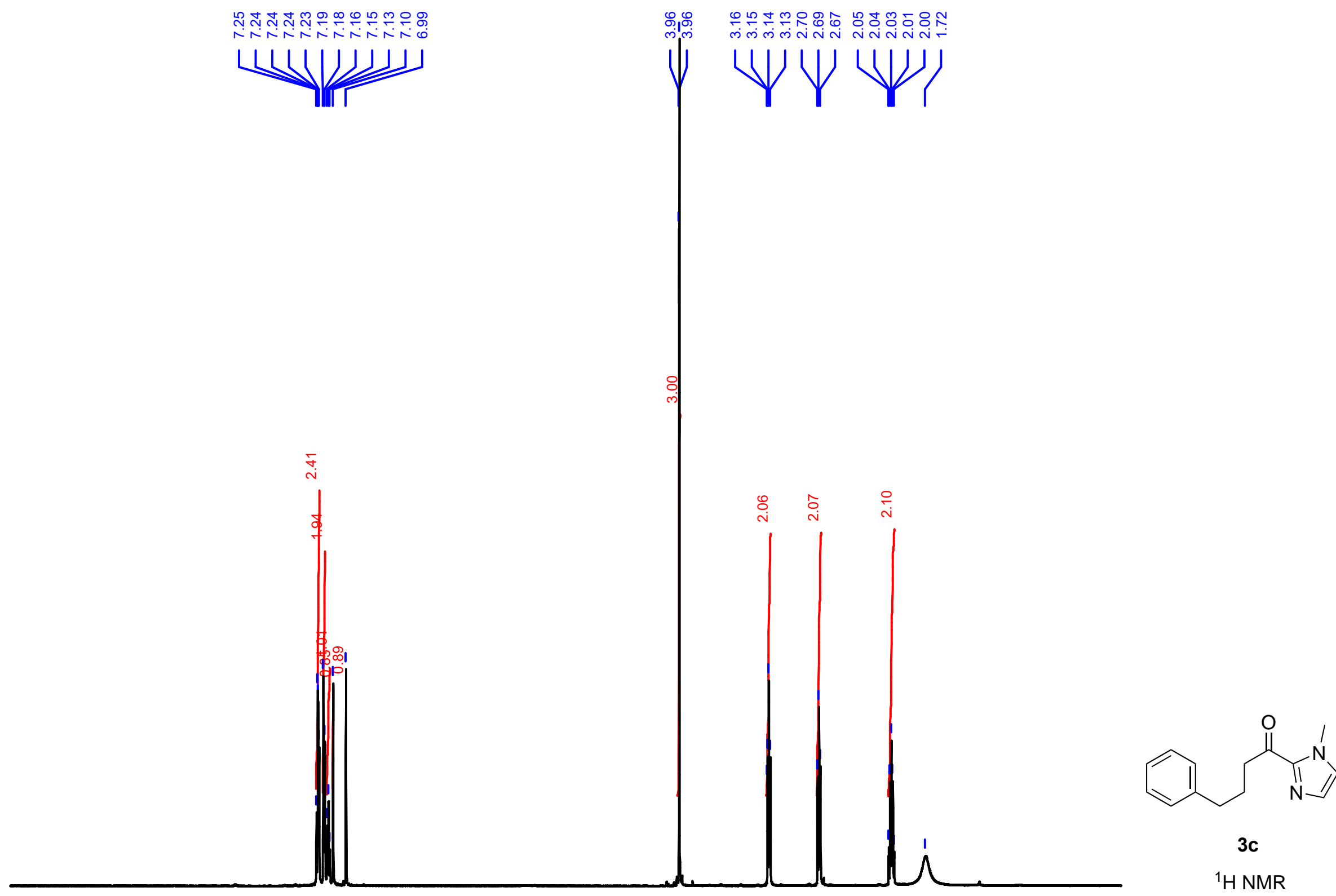


$$
H_{1}
$$






WWU1

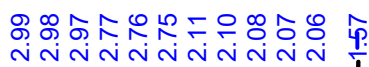

4 ll

WW
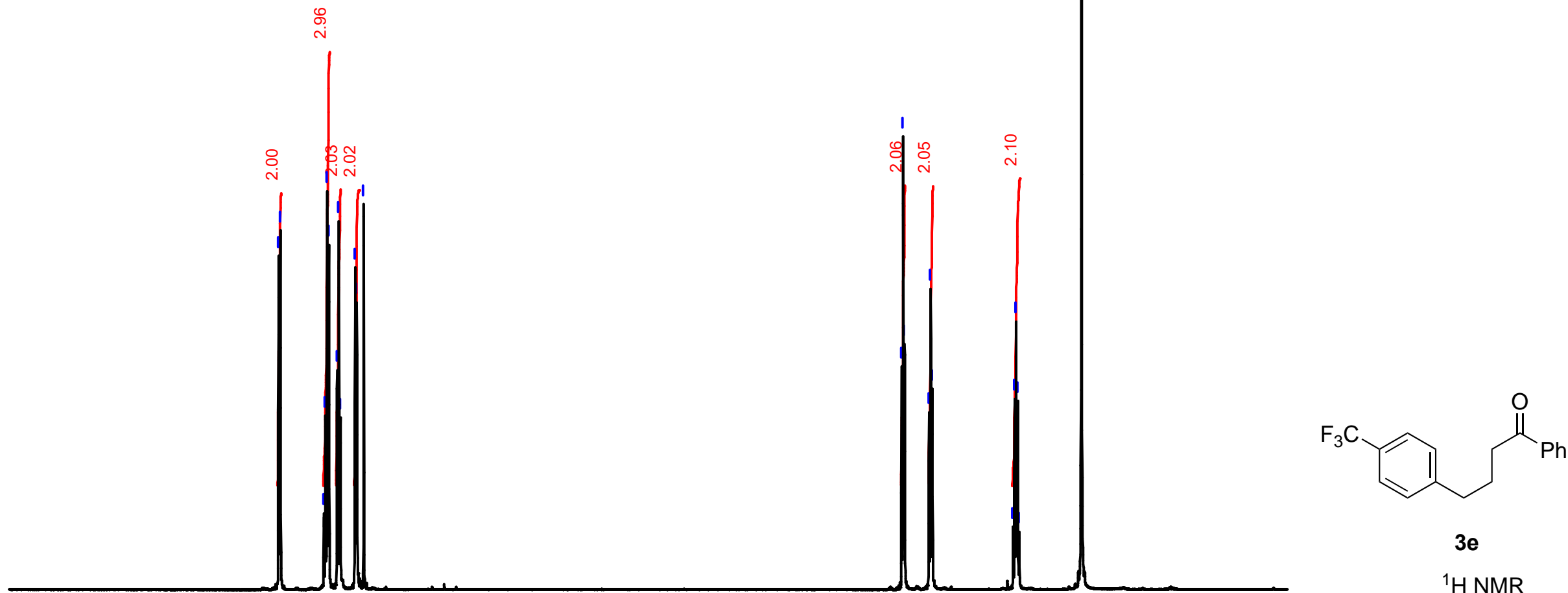

$3 e$

${ }^{1} \mathrm{H}$ NMR

(600 MHz, $\mathrm{CDCl}_{3}$ ) 

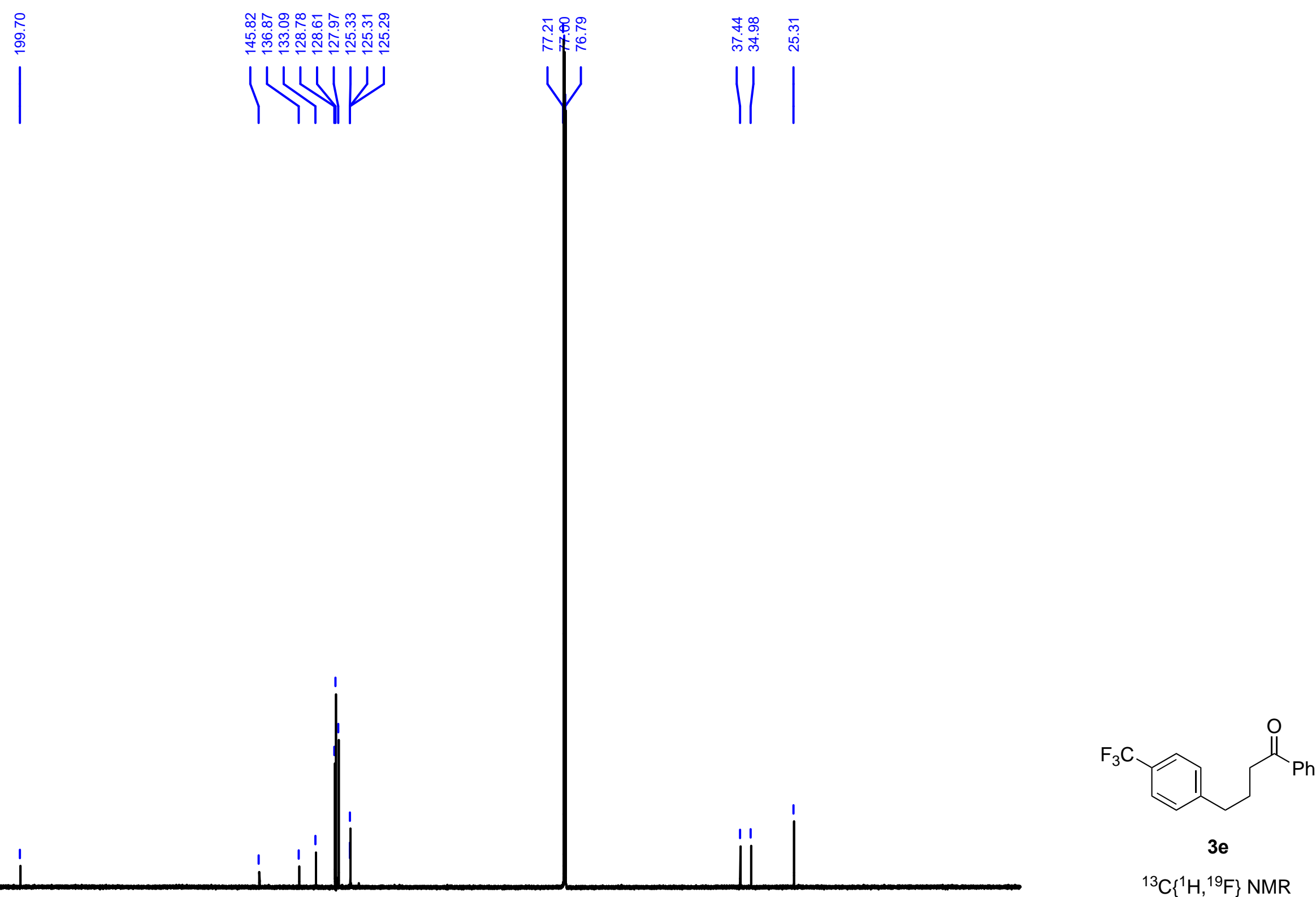

${ }^{13} \mathrm{C}\left\{{ }^{1} \mathrm{H},{ }^{19} \mathrm{~F}\right\}$ NMR

$\left(150 \mathrm{MHz}, \mathrm{CDCl}_{3}\right)$

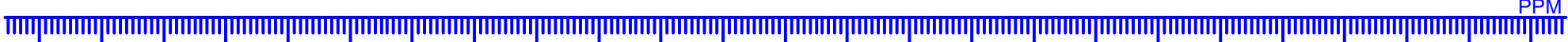
$\begin{array}{lllllllllllllllllllllllll}220 & 210 & 200 & 190 & 180 & 170 & 160 & 150 & 140 & 130 & 120 & 110 & 100 & 90 & 80 & 70 & 60 & 50 & 40 & 30 & 20 & 10 & 0 & -10 & -20\end{array}$ 


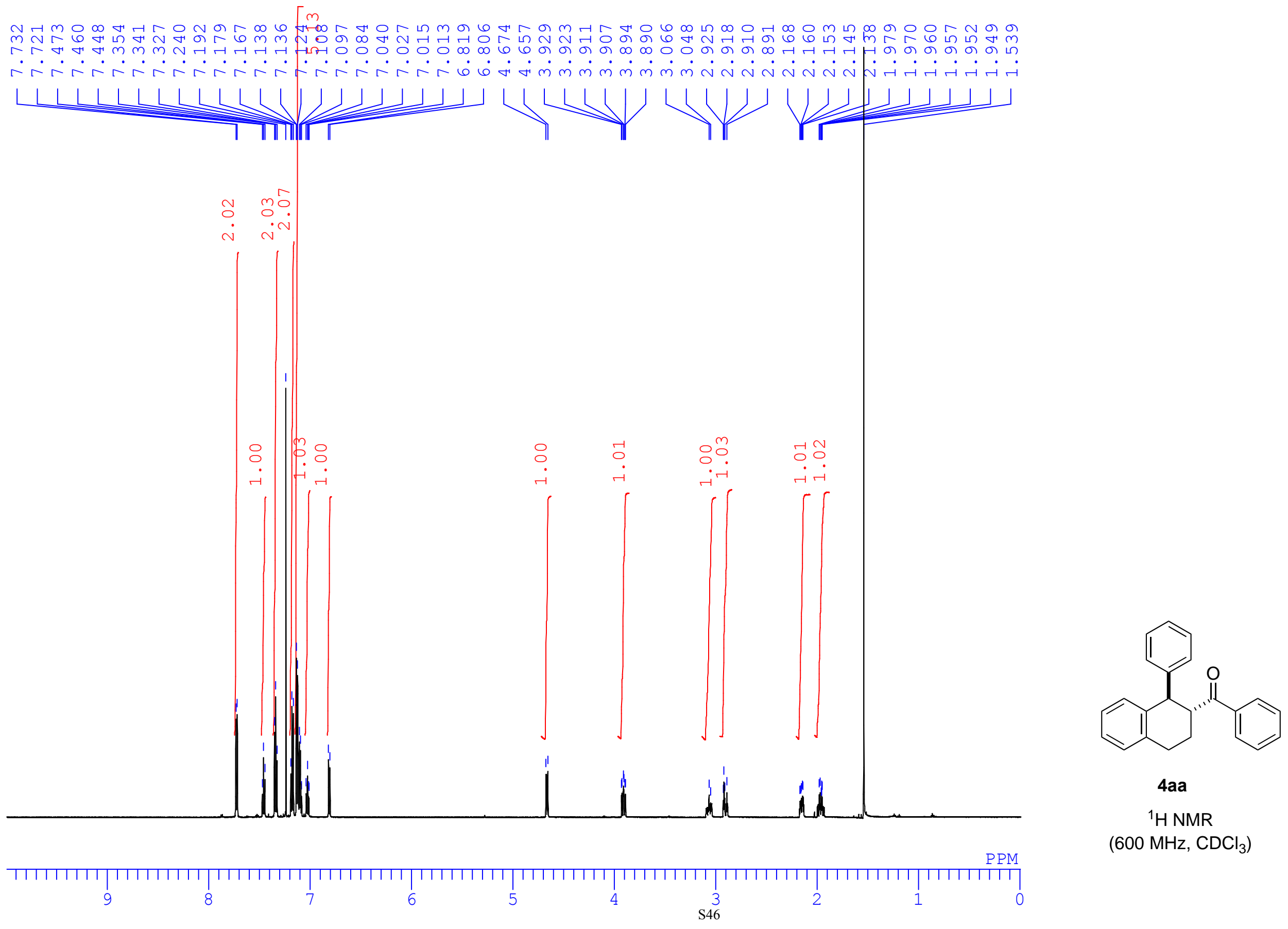




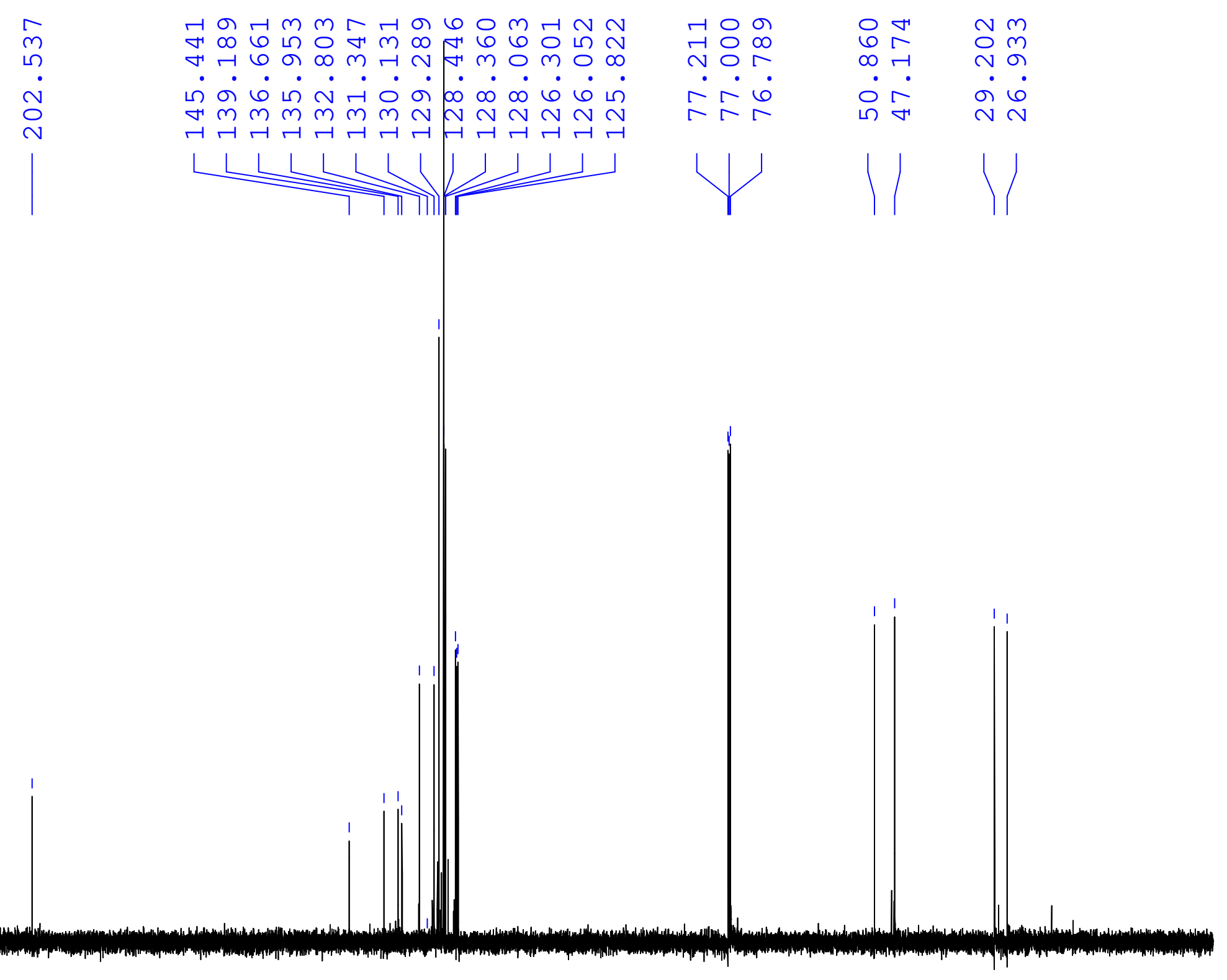

PPM

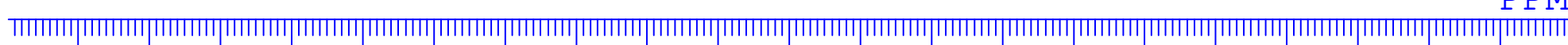

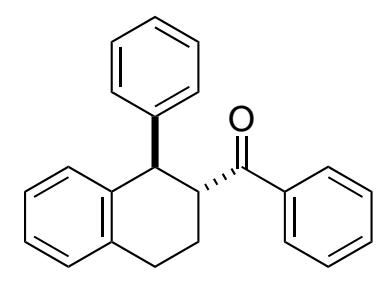

4aa

${ }^{13} \mathrm{C}\left\{{ }^{1} \mathrm{H}\right\}$ NMR

$\left(150 \mathrm{MHz}, \mathrm{CDCl}_{3}\right)$ 


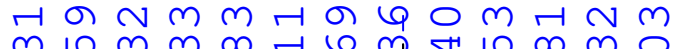
$m \backsim m m \infty$

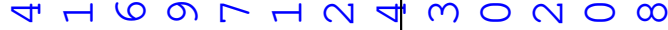
$\dot{0} \dot{0} \dot{\sim} \dot{0} \propto \dot{\sigma} \infty \dot{\omega} \dot{\omega} \dot{0}$

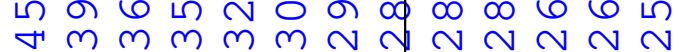

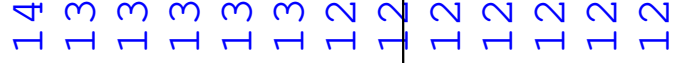

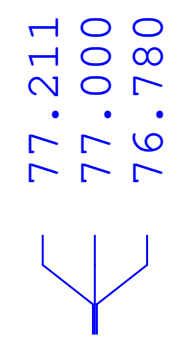

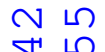

ॠ

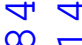

$\checkmark$

N $O$

तन

$\sim$

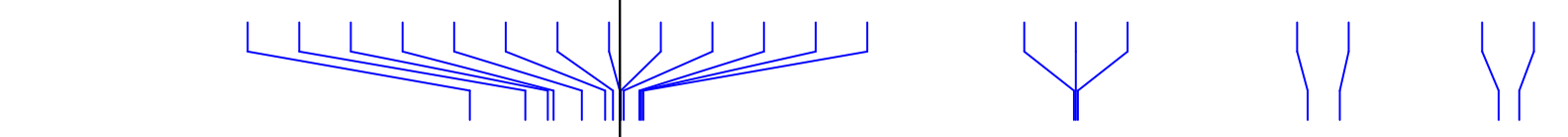

O

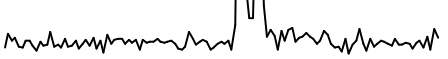

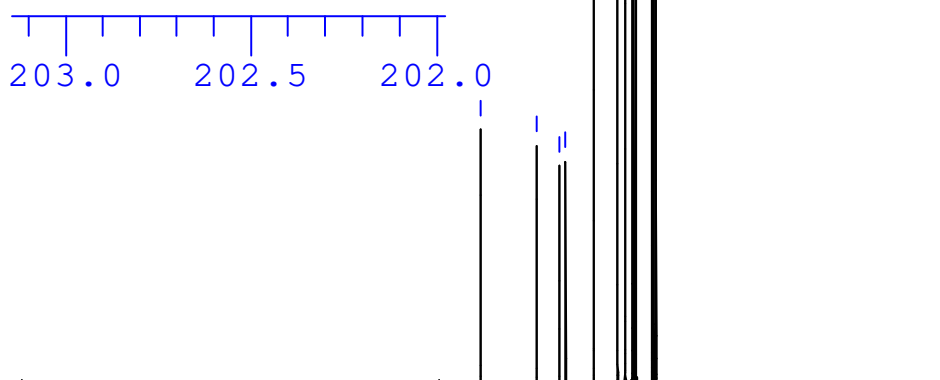

203.0

202.5

202.0
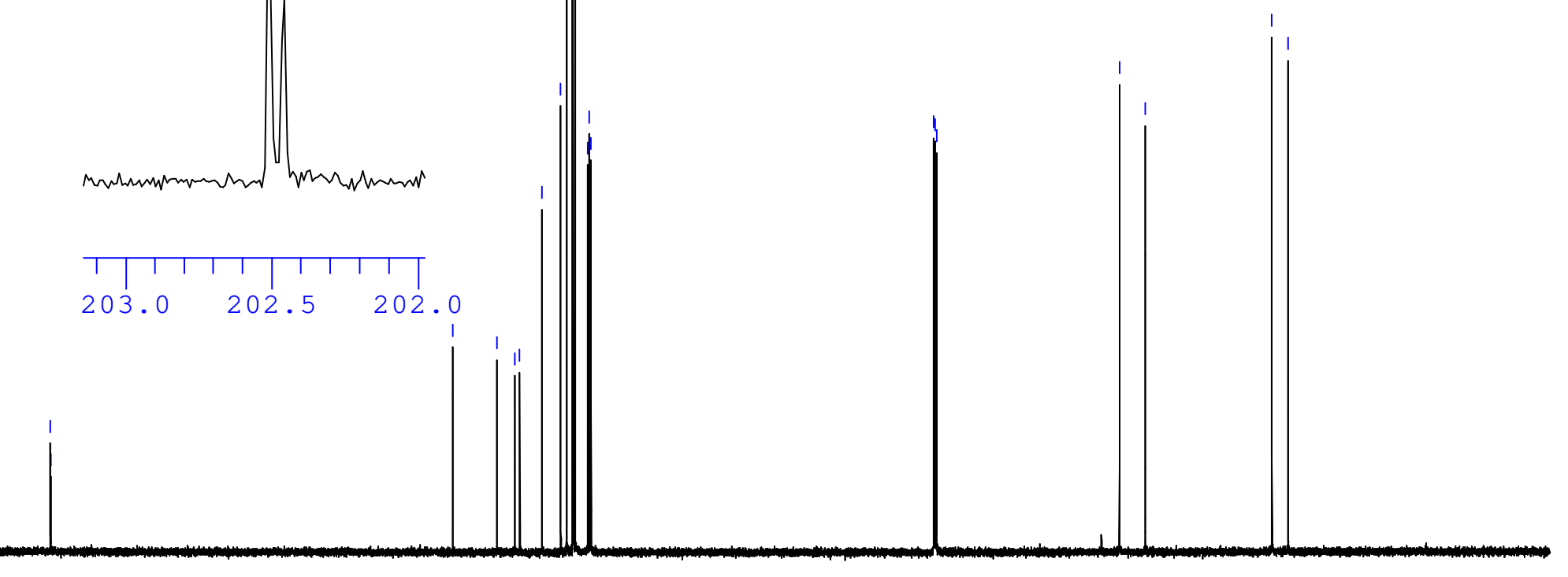

PPM

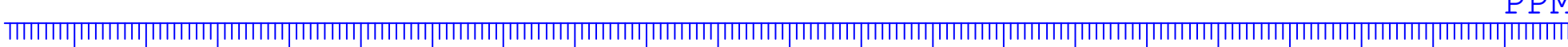

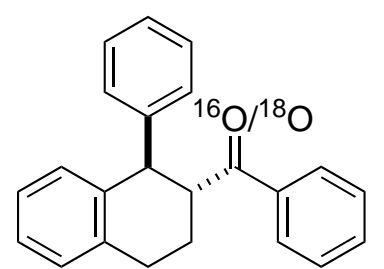

पDD—DD 2

${ }^{13} \mathrm{C}\left\{{ }^{1} \mathrm{H}\right\} \mathrm{NMR}$

$\left(150 \mathrm{MHz}, \mathrm{CDCl}_{3}\right.$ ) 


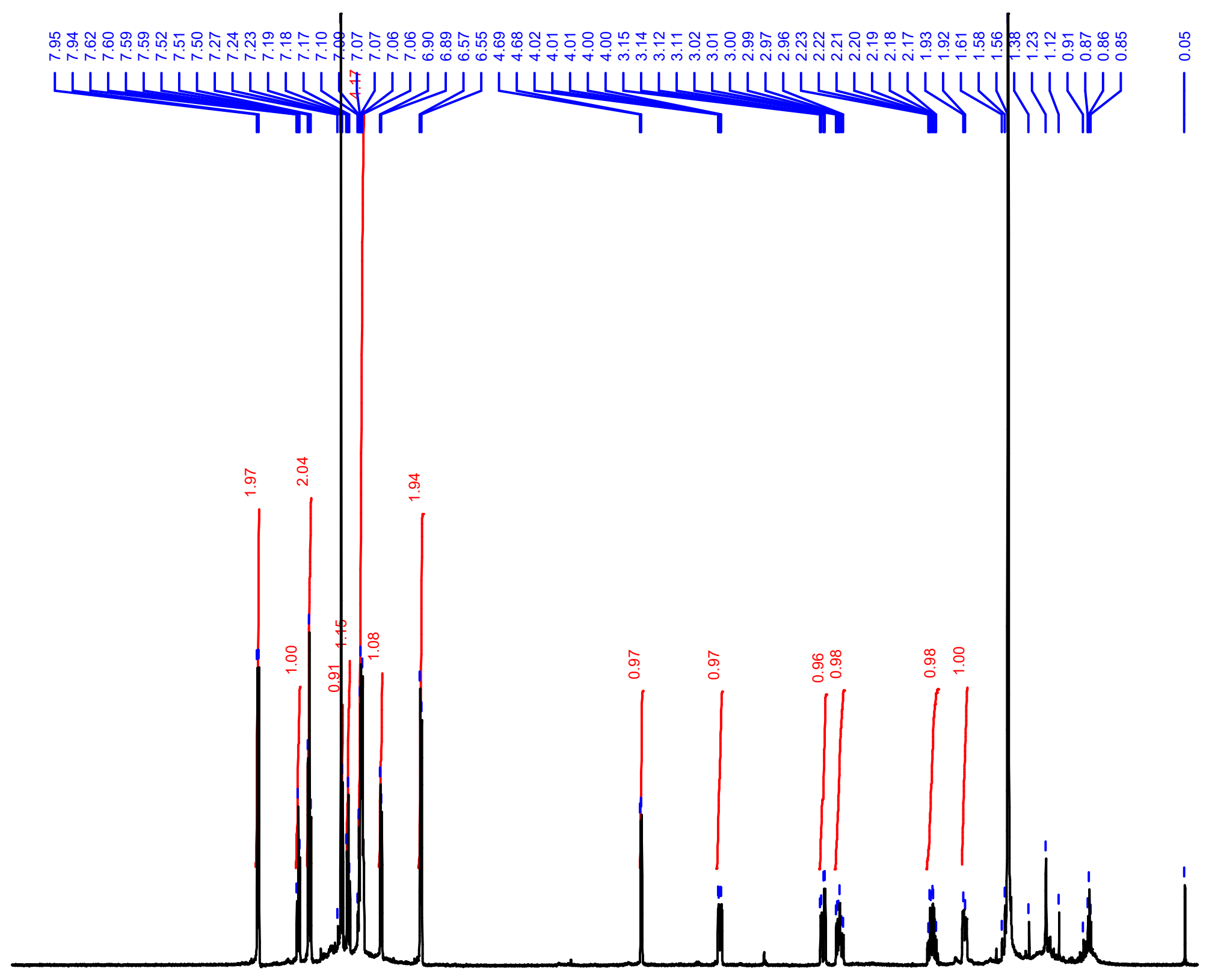

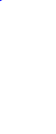





TाTाTा

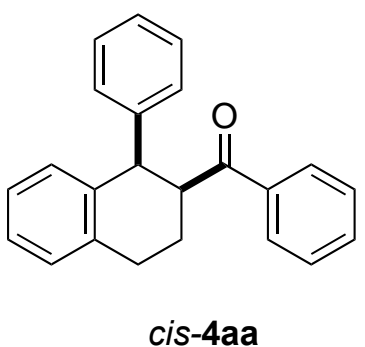

${ }^{13} \mathrm{C}\left\{{ }^{1} \mathrm{H}\right\}$ NMR $\left(150 \mathrm{MHz}, \mathrm{CDCl}_{3}\right)$

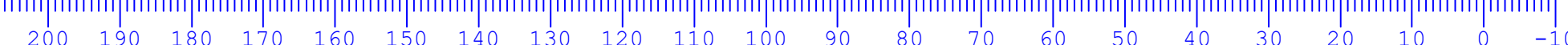




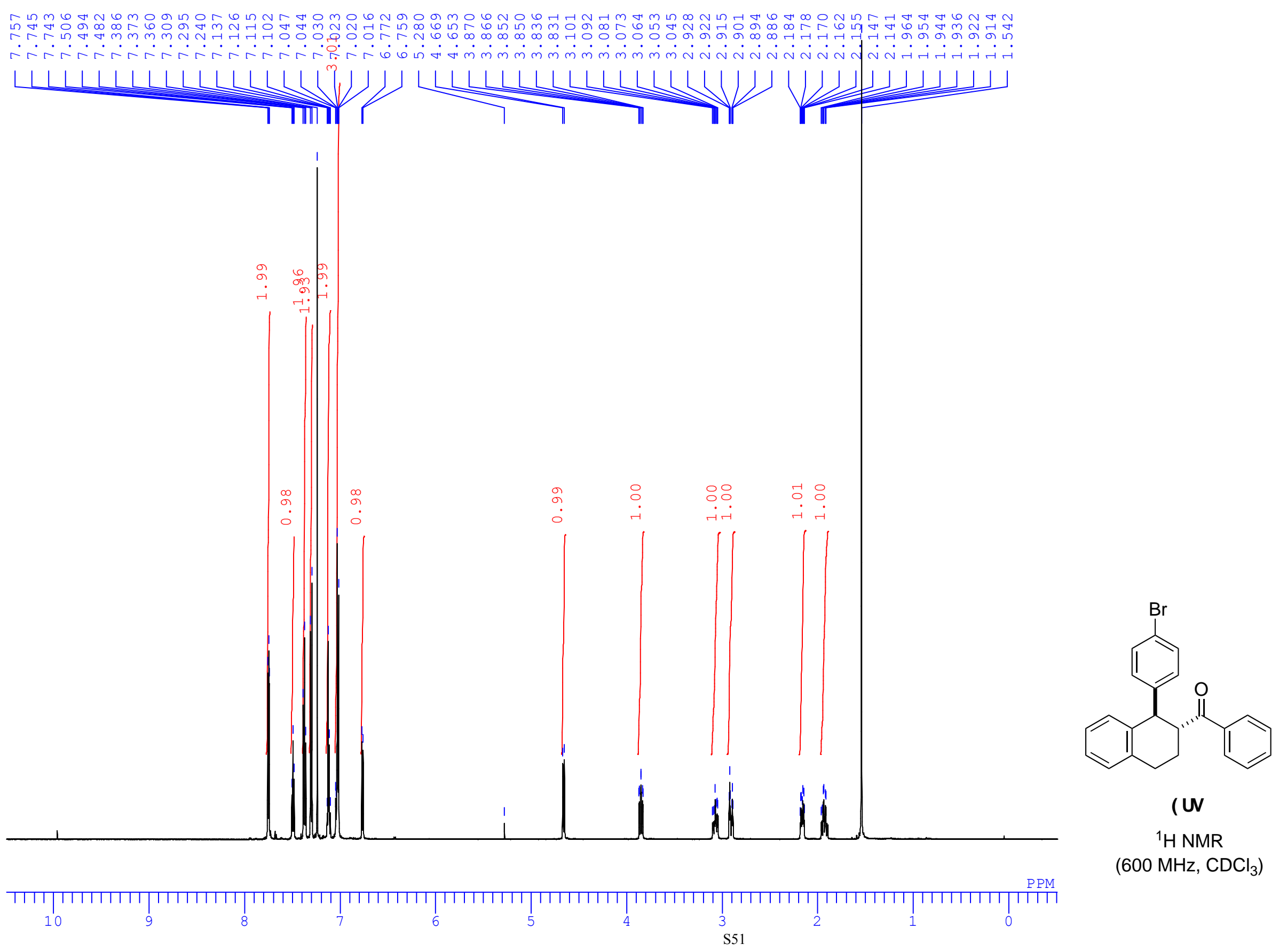




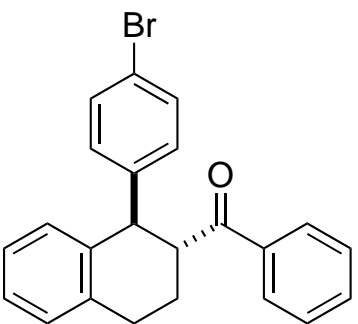

DE

${ }^{13} \mathrm{C}\left\{{ }^{1} \mathrm{H}\right\}$ NMR $\left(150 \mathrm{MHz}, \mathrm{CDCl}_{3}\right)$ 
당ㅇ $6 m$ の

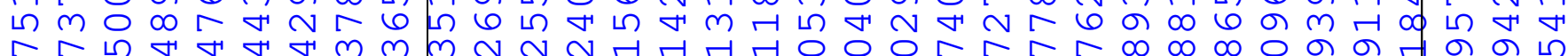

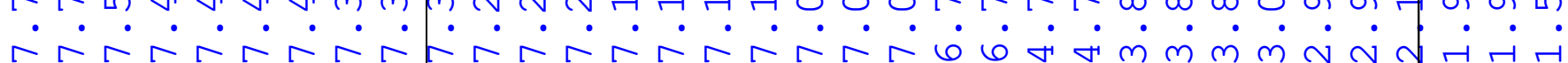

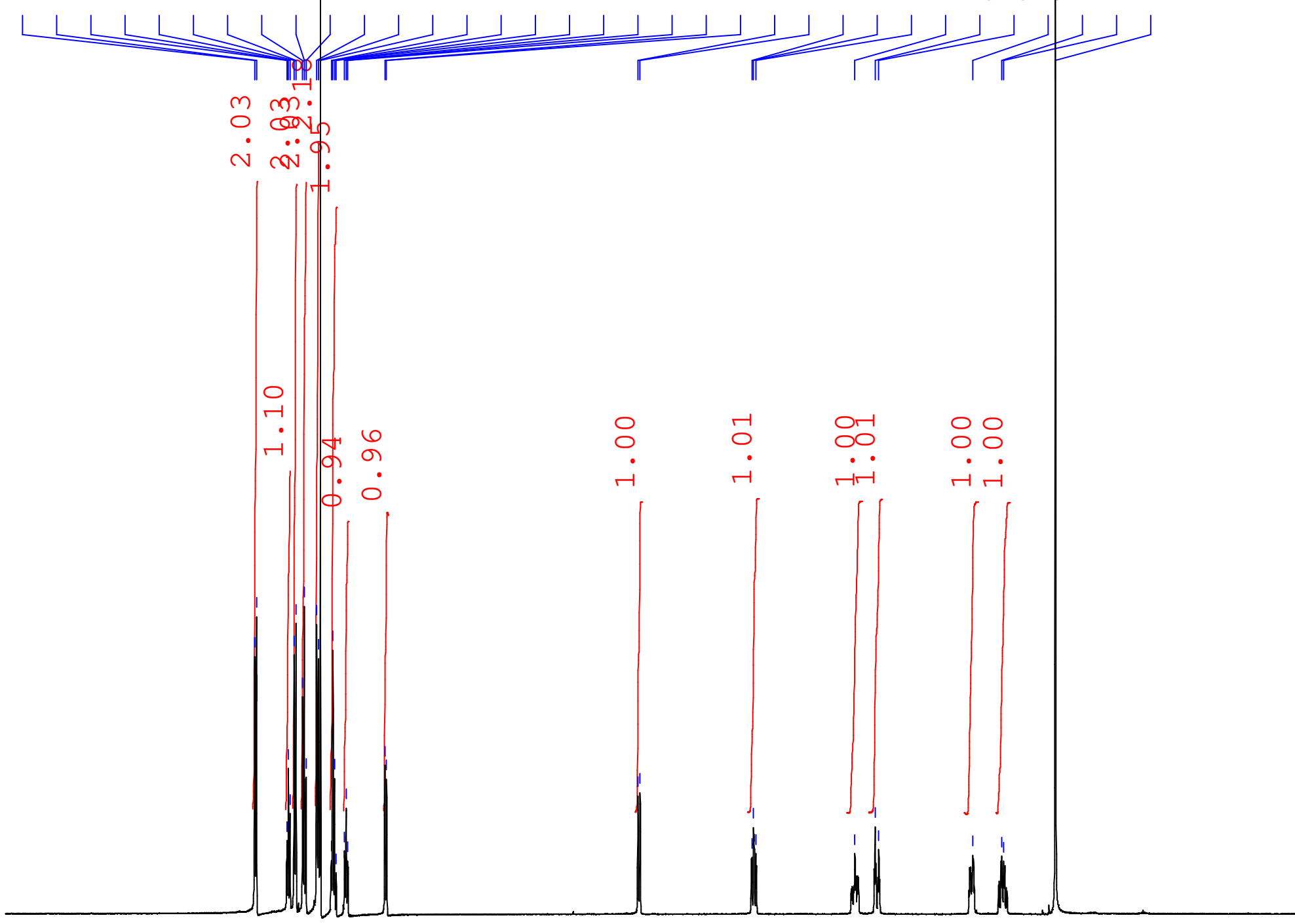

PPM

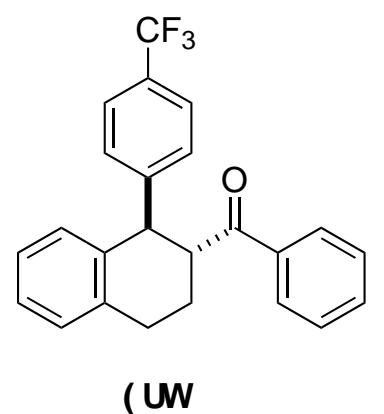

${ }^{1} \mathrm{H}$ NMR

(600 MHz, $\mathrm{CDCl}_{3}$ ) 

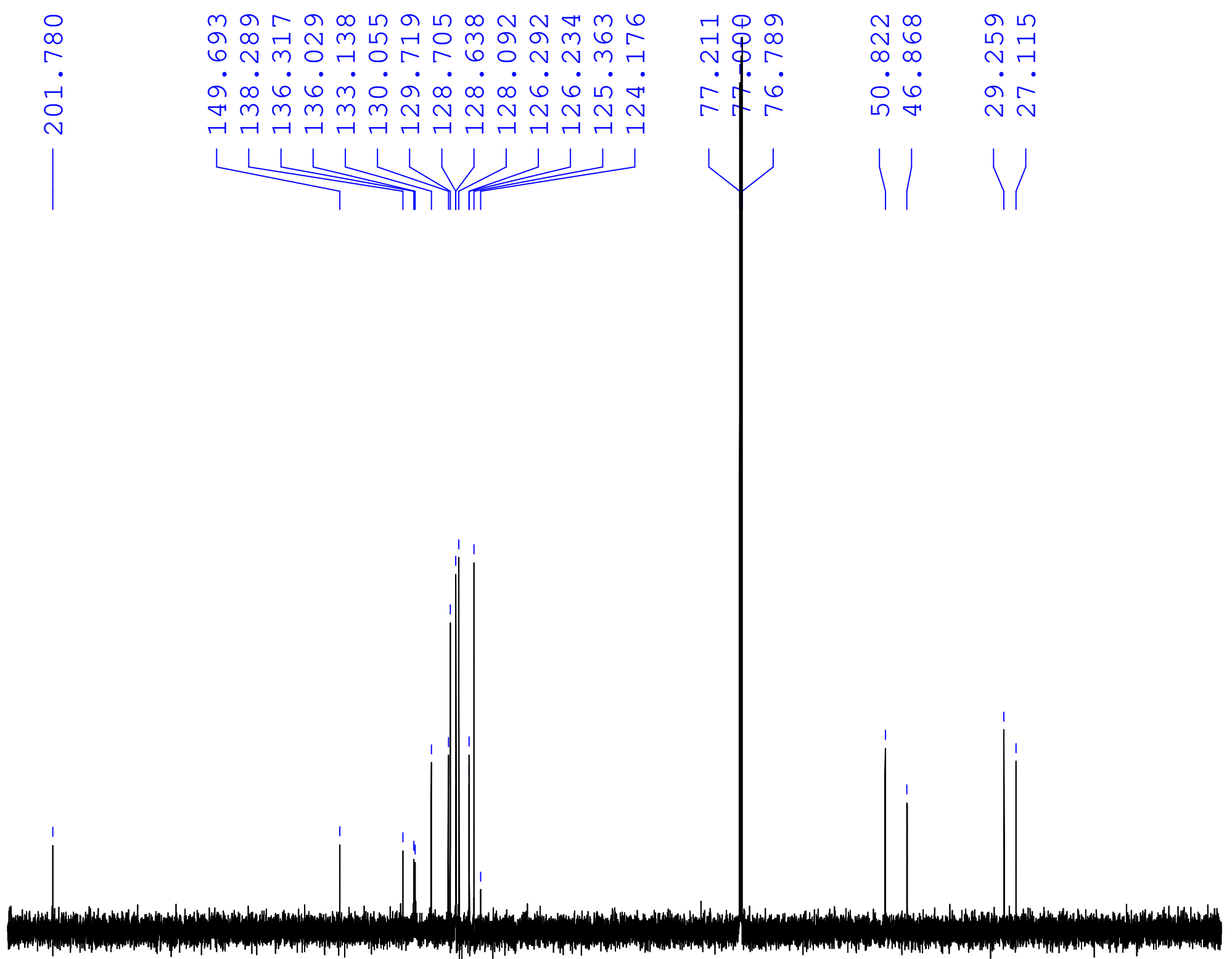

PPM

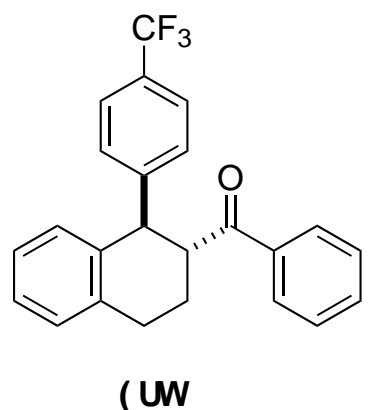

${ }^{13} \mathrm{C}\left\{{ }^{1} \mathrm{H},{ }^{19} \mathrm{~F}\right\}$ NMR $\left(150 \mathrm{MHz}, \mathrm{CDCl}_{3}\right)$ 


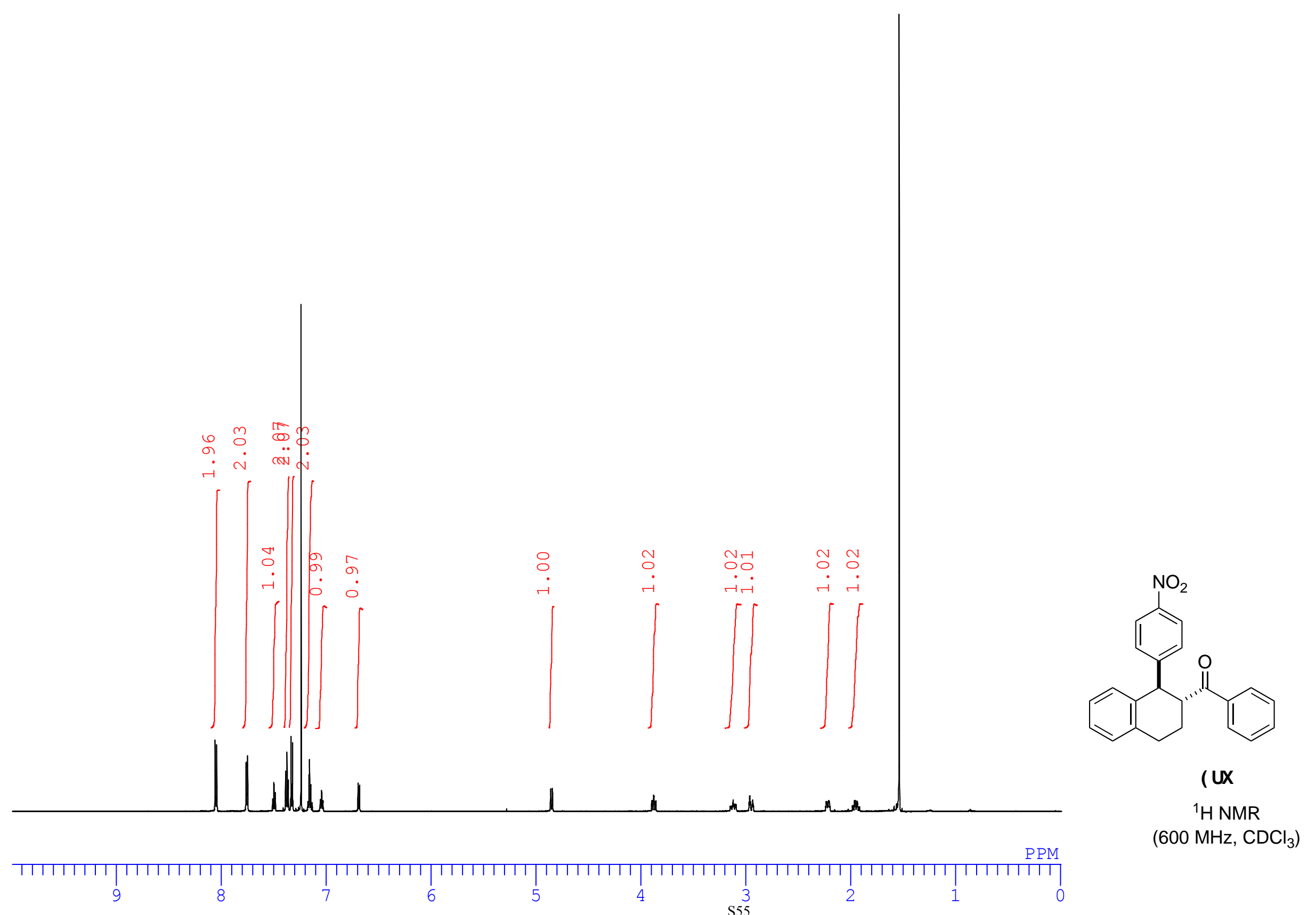




$$
\underbrace{\prime \prime}_{4}
$$




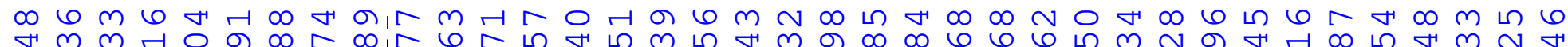
অm $m$ 궁

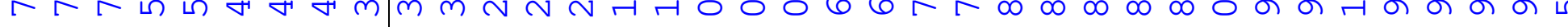

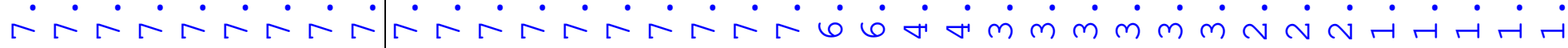

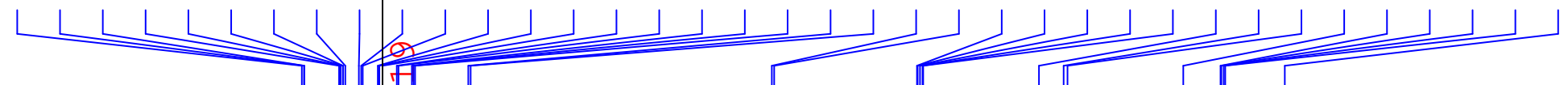

$11 / \sqrt{11}$

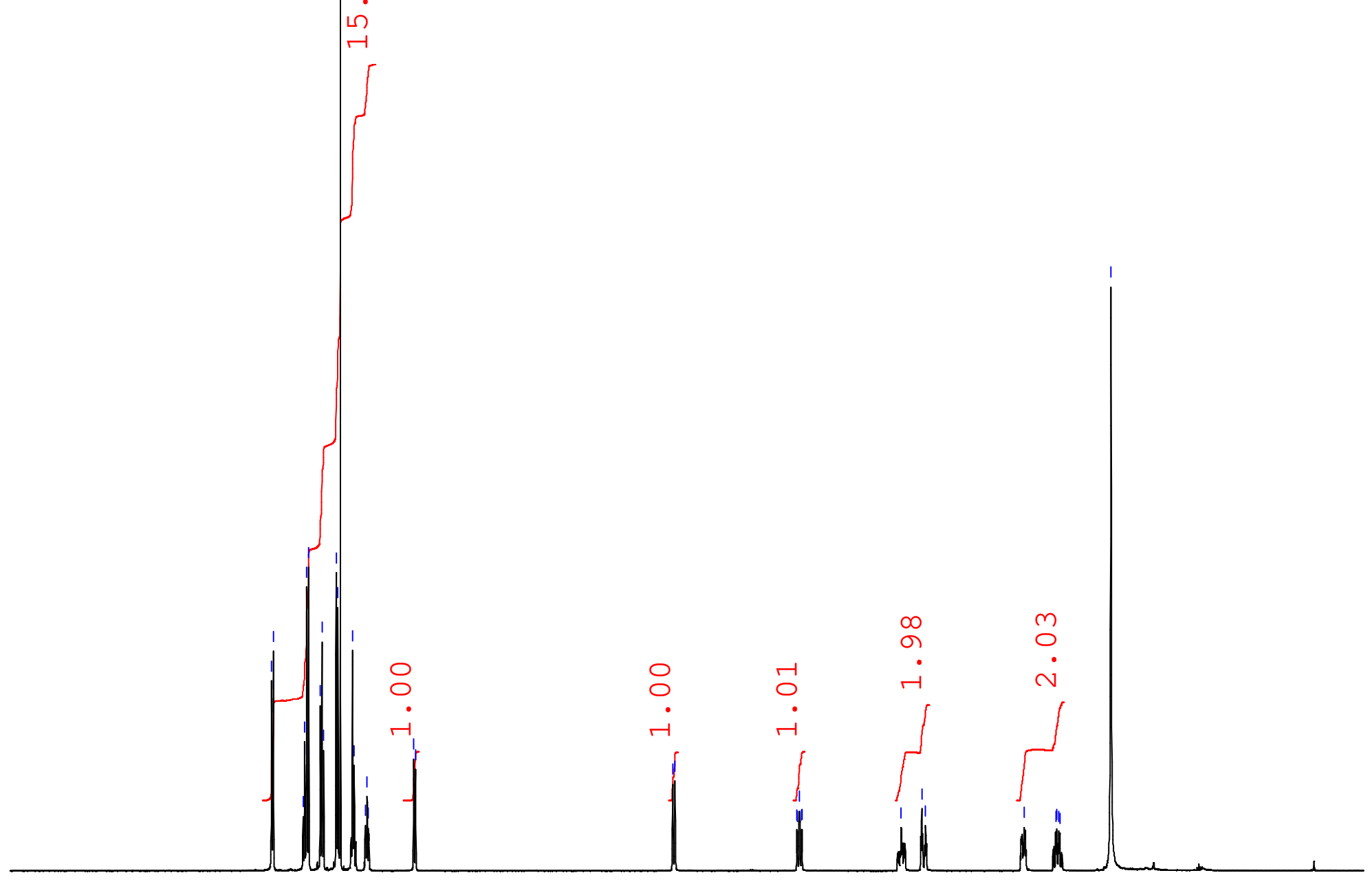

PPM

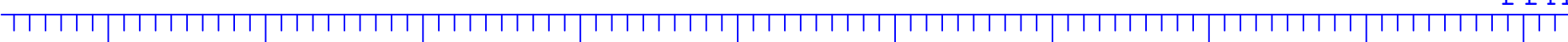

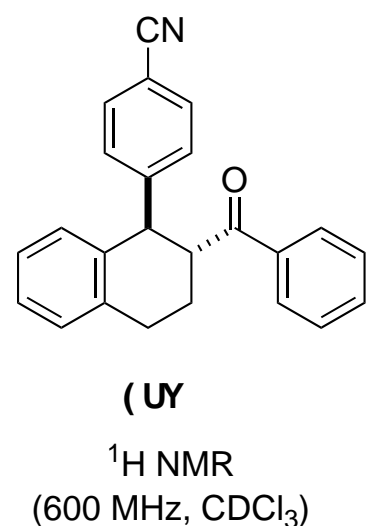




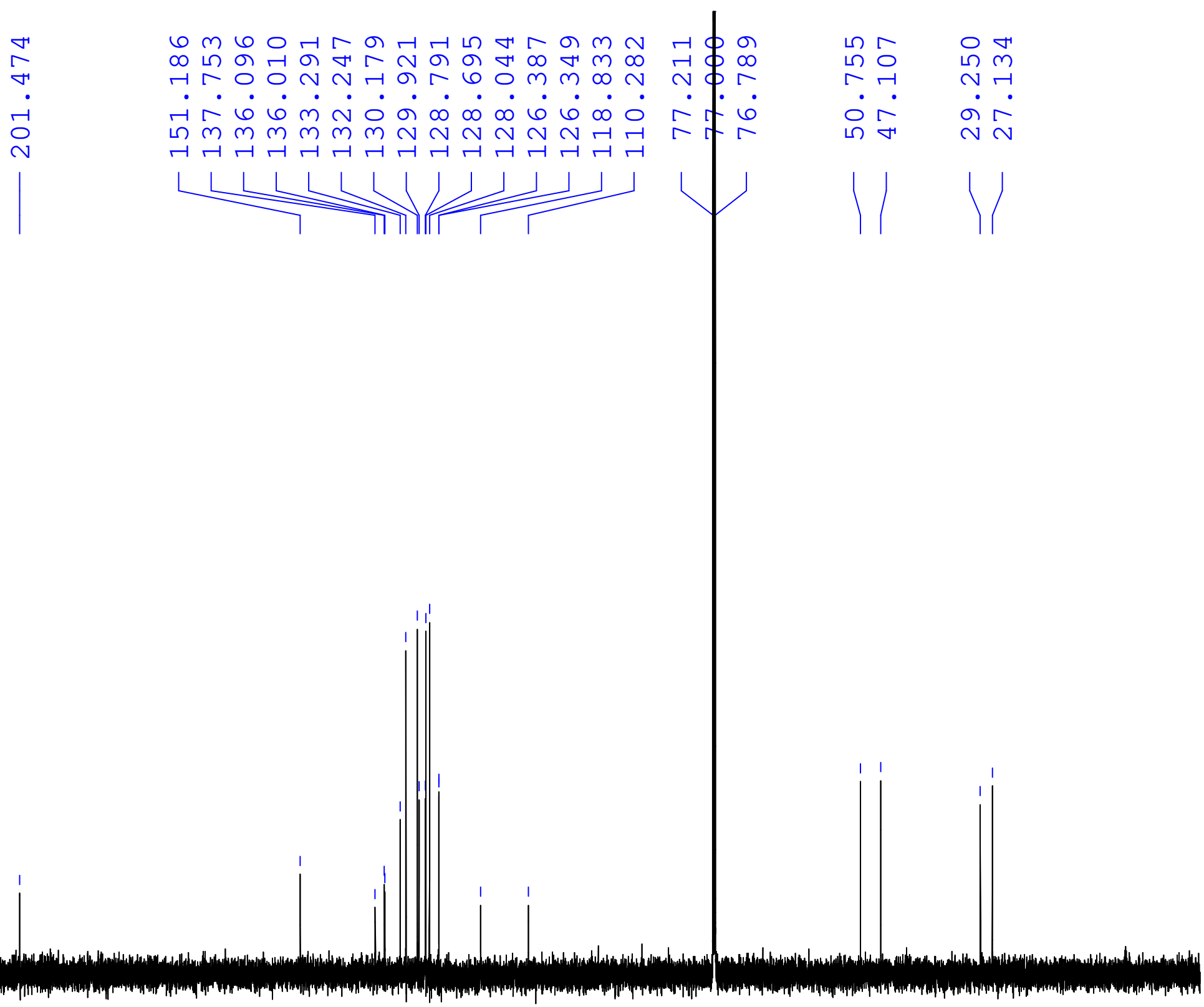

PPM

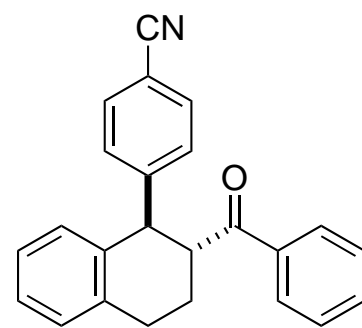

DH

${ }^{13} \mathrm{C}\left\{{ }^{1} \mathrm{H}\right\}$ NMR $\left(150 \mathrm{MHz}, \mathrm{CDCl}_{3}\right.$ ) 


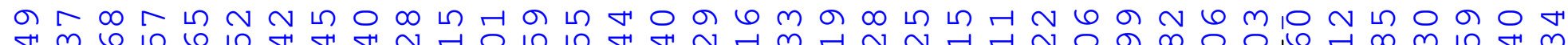

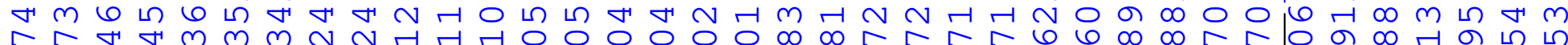

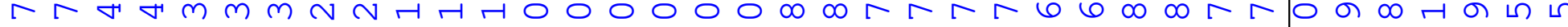

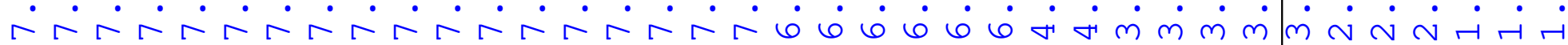
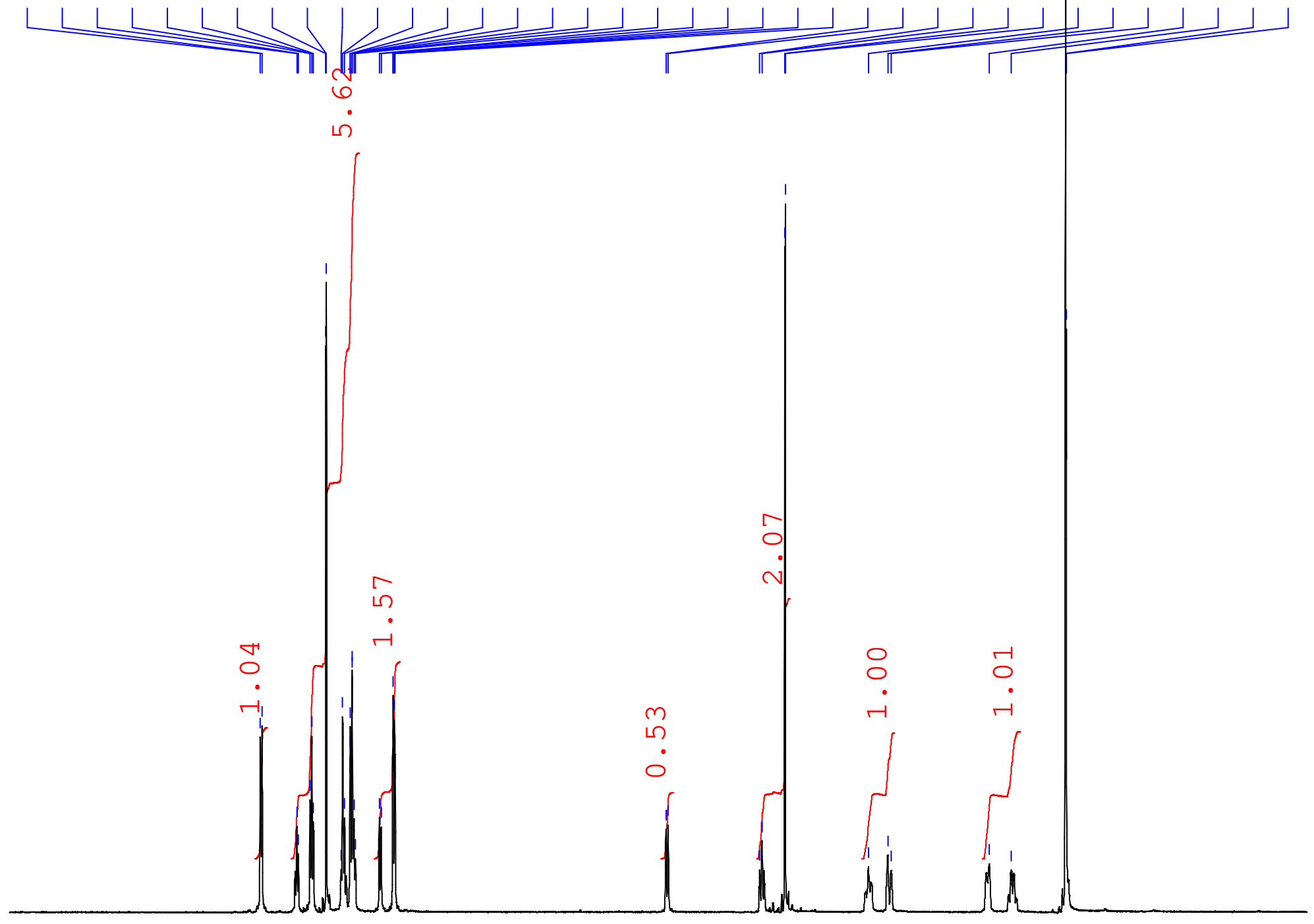

PPM

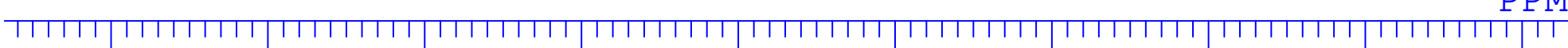








PPM

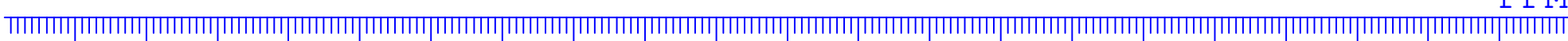

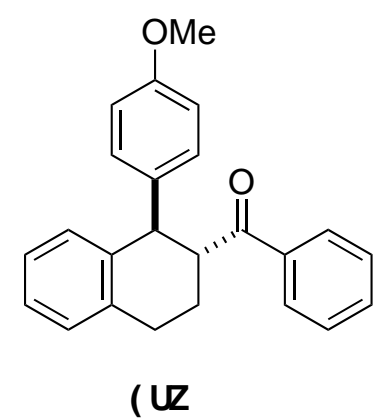

${ }^{13} \mathrm{C}\left\{{ }^{1} \mathrm{H}\right\} \mathrm{NMR}$ $\left(150 \mathrm{MHz}, \mathrm{CDCl}_{3}\right)$ 


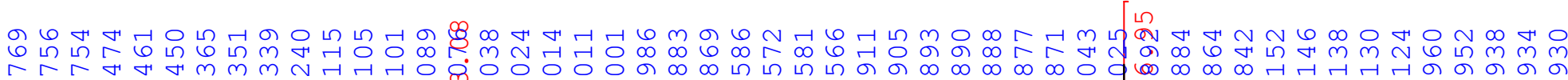

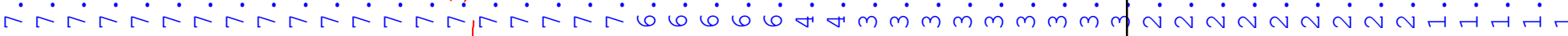

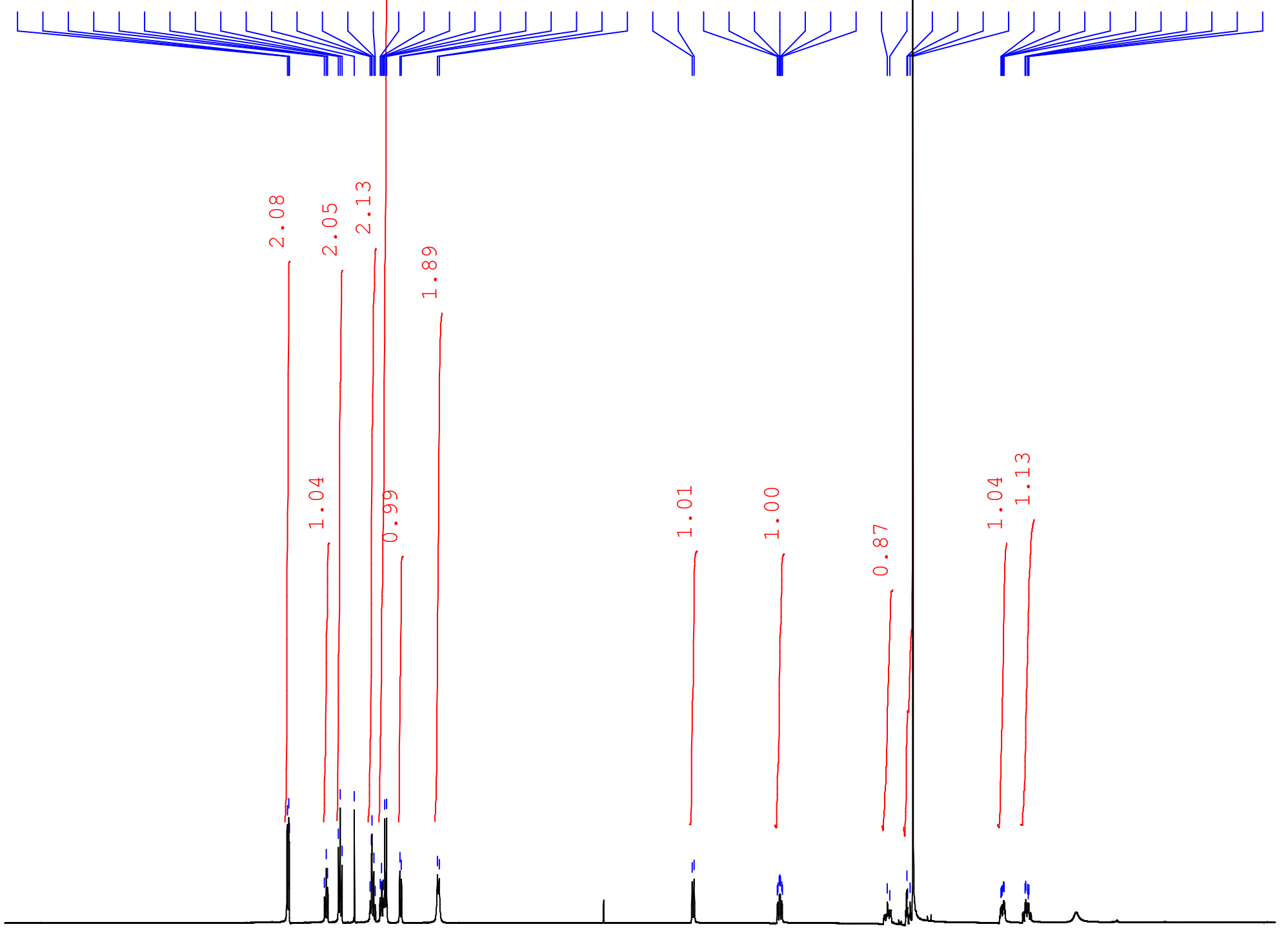

PPM

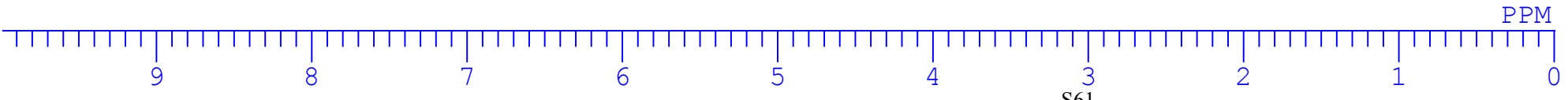

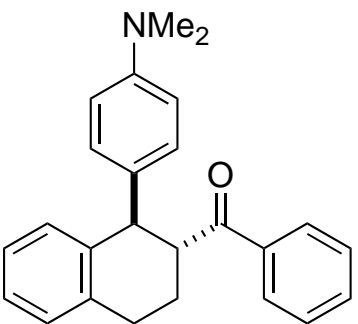

૧DJ

${ }^{1} \mathrm{H}$ NMR

$\left(600 \mathrm{MHz}, \mathrm{CDCl}_{3}\right.$ ) 


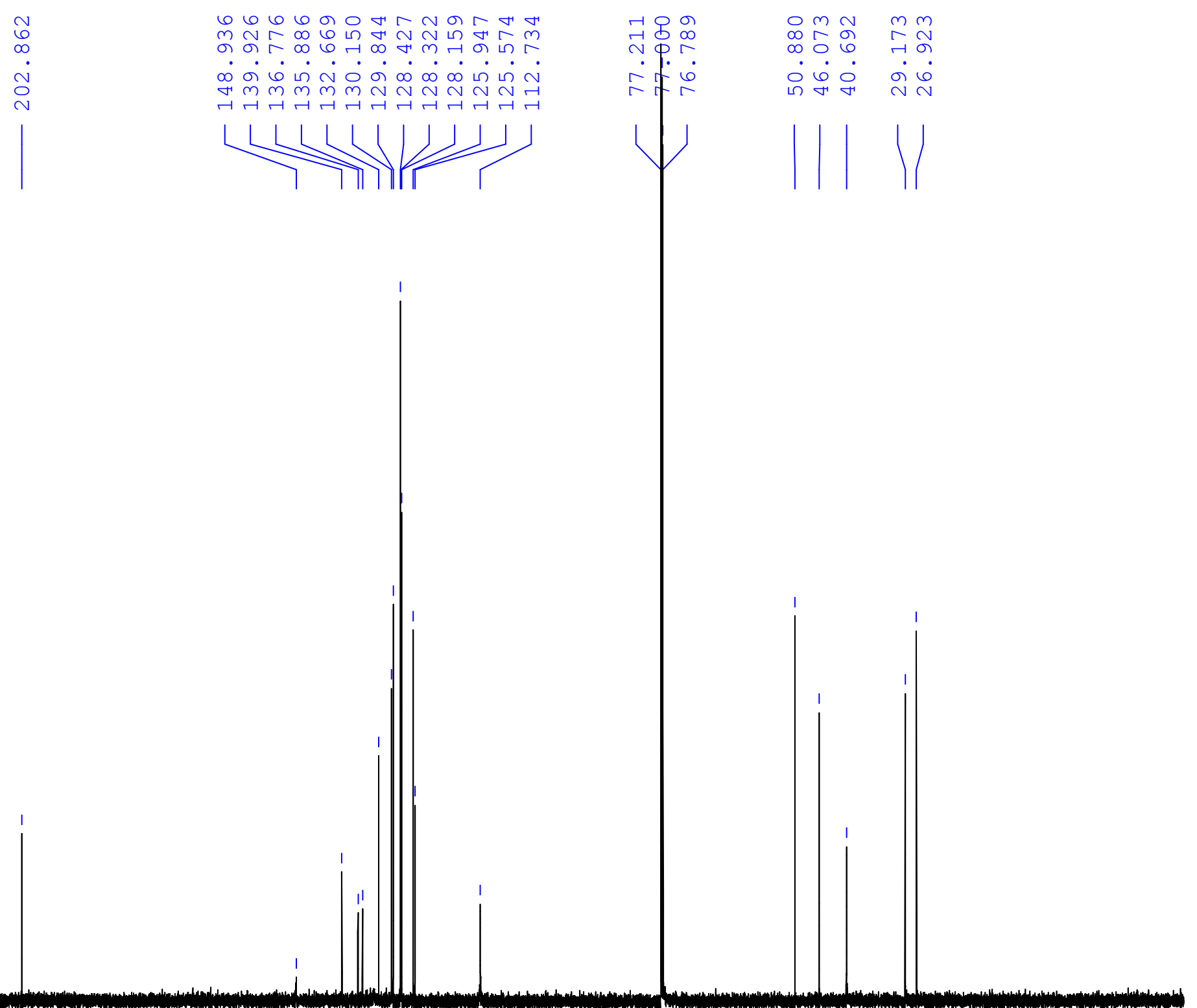

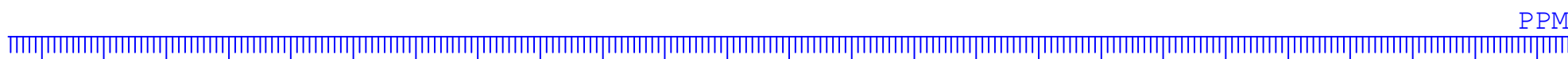

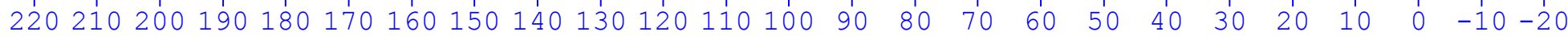

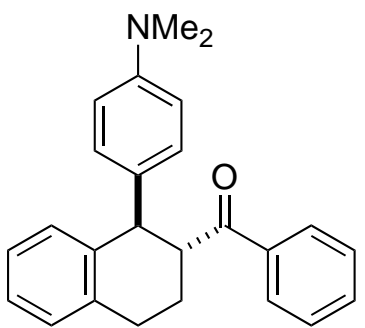

$\square \mathrm{DJ}$

${ }^{13} \mathrm{C}\left\{{ }^{1} \mathrm{H}\right\}$ NMR $\left(150 \mathrm{MHz}, \mathrm{CDCl}_{3}\right)$ 


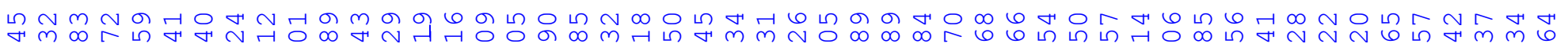
सM⿻

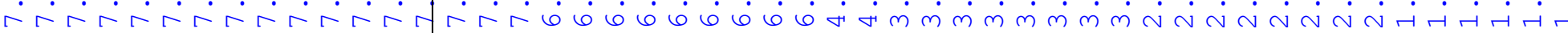

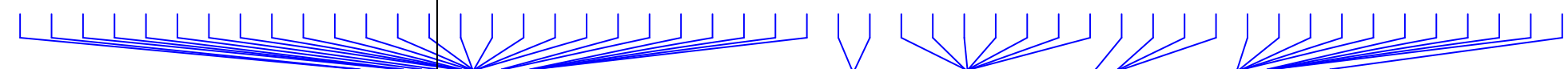
177 तो
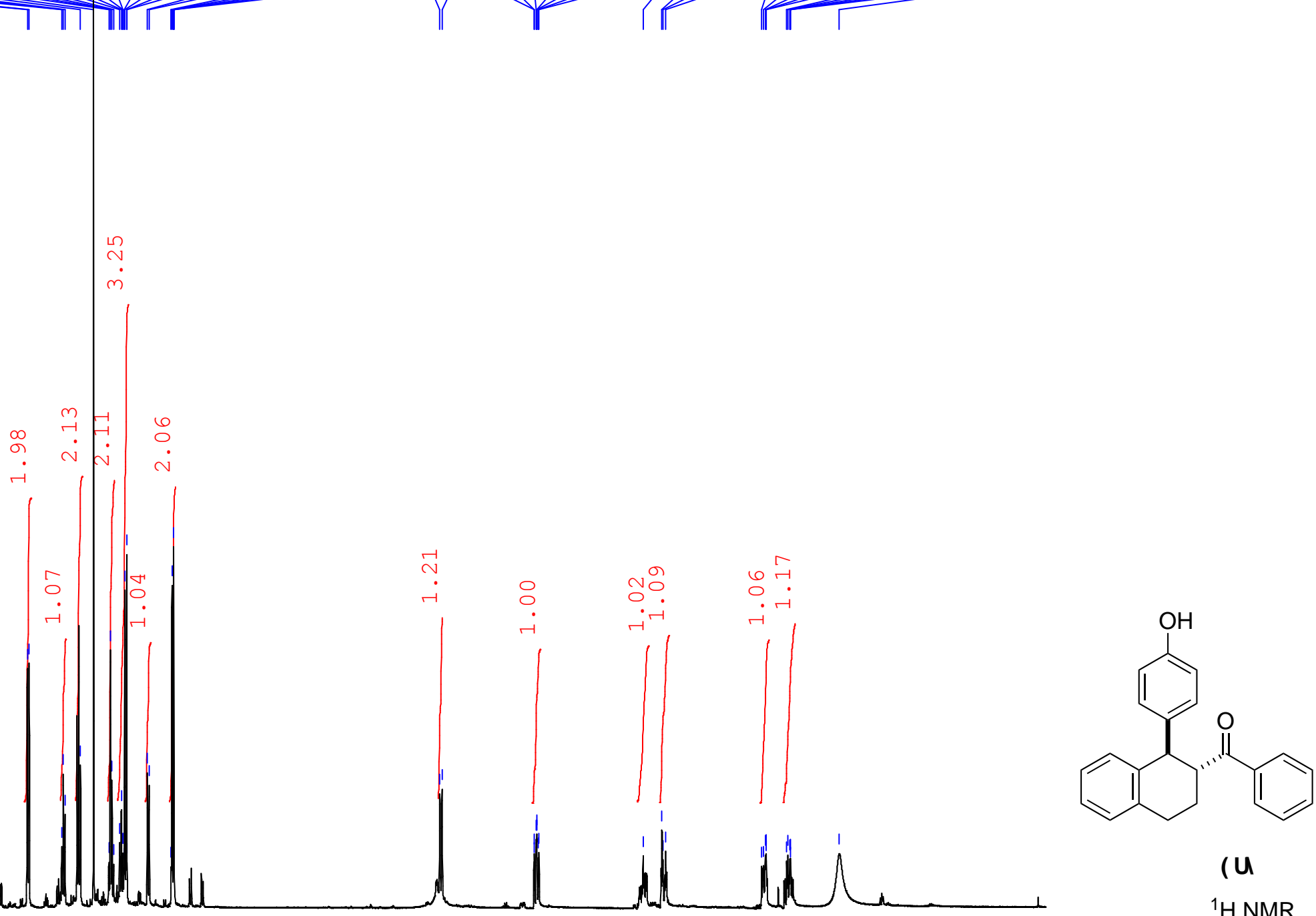

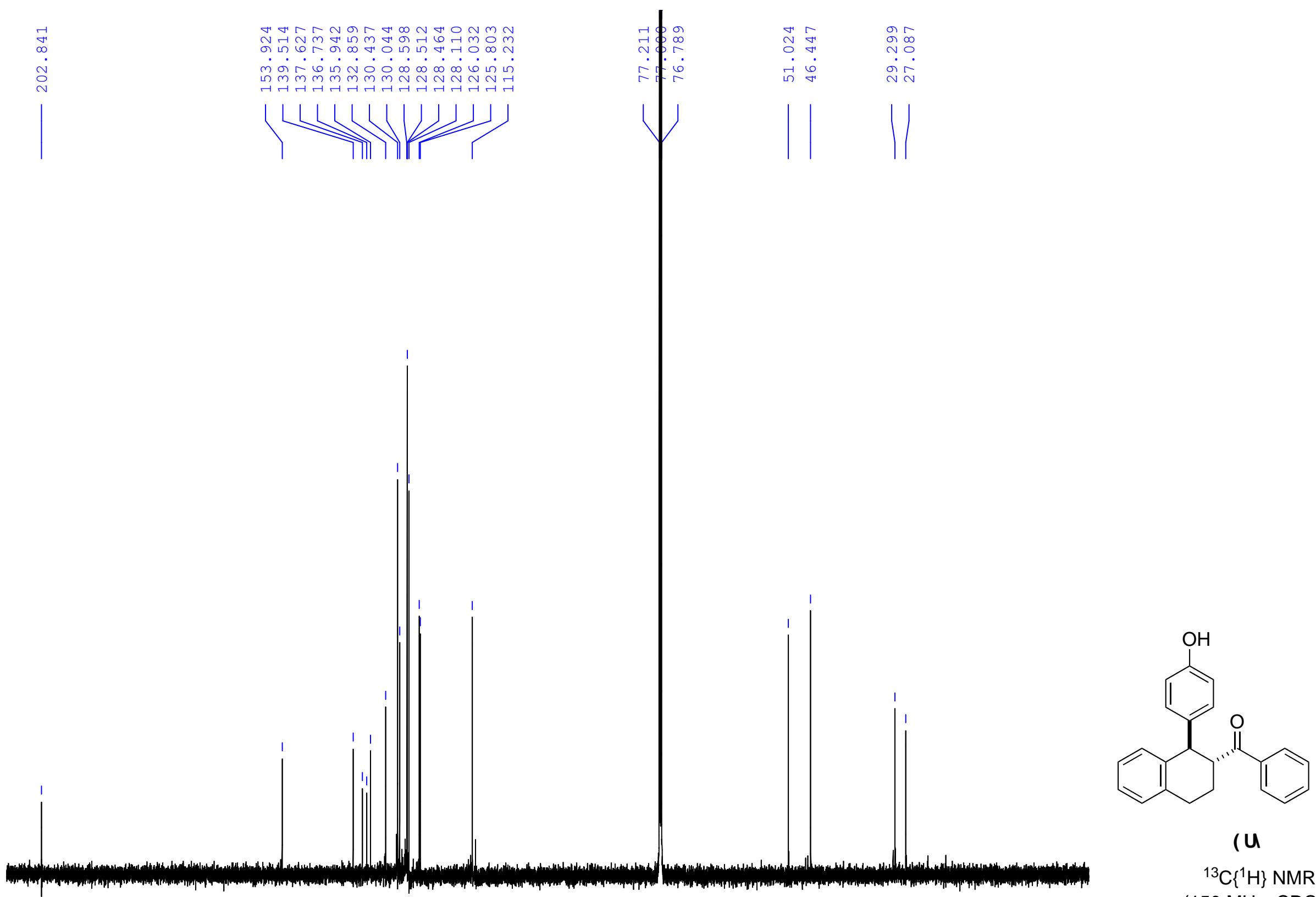

${ }^{13} \mathrm{C}\left\{{ }^{1} \mathrm{H}\right\}$ NMR $\left(150 \mathrm{MHz}, \mathrm{CDCl}_{3}\right)$

Tापा






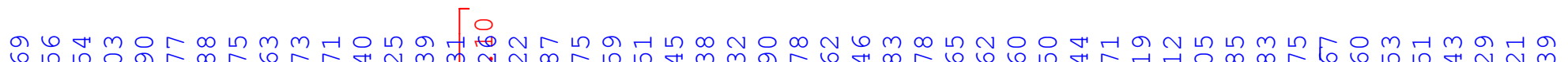

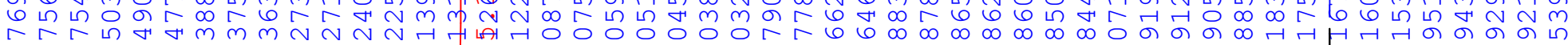

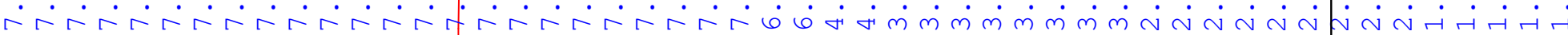
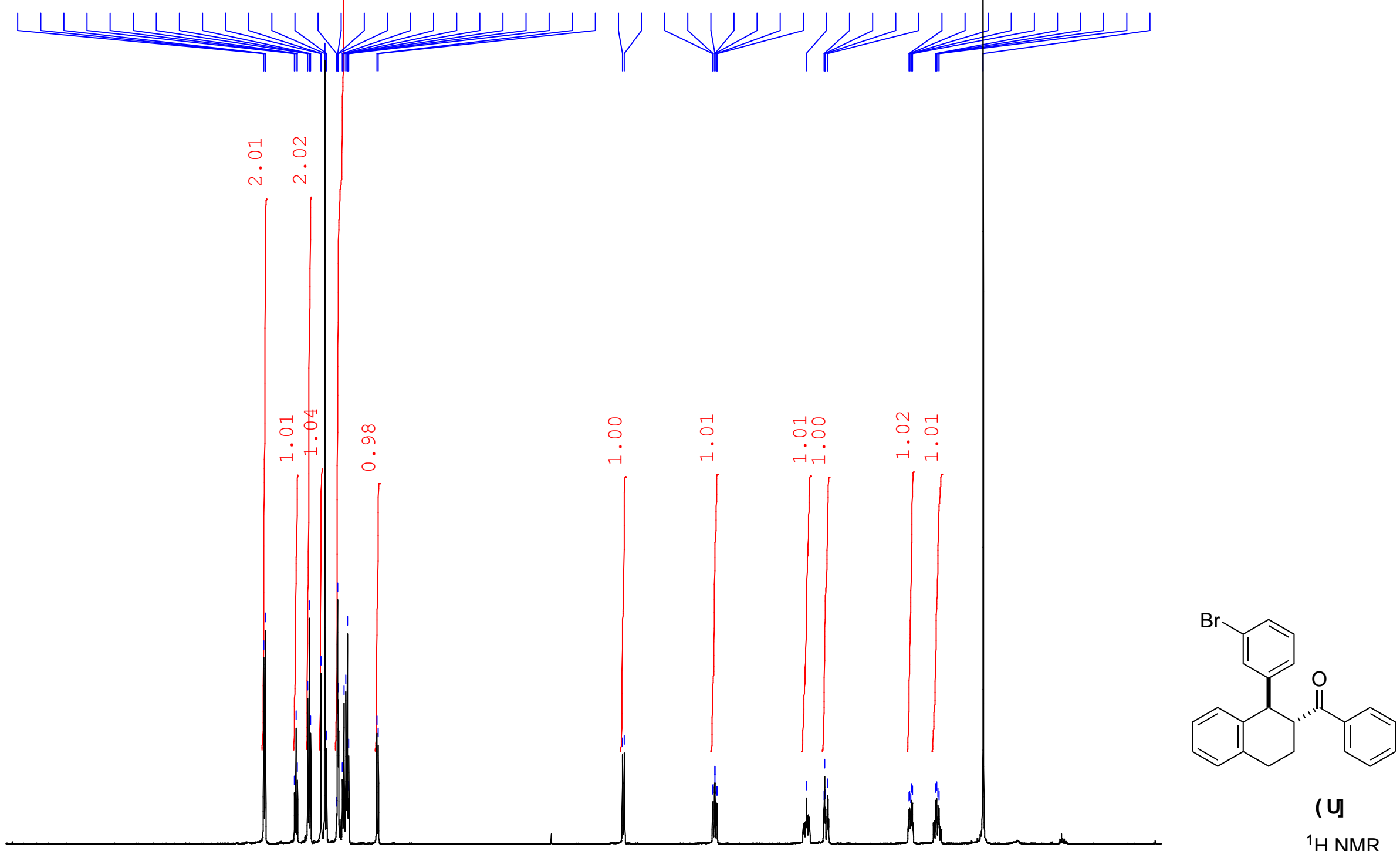

$\square \mathbf{D L}$

${ }^{1} \mathrm{H}$ NMR $\left(600 \mathrm{MHz}, \mathrm{CDCl}_{3}\right)$ 


$$
\text { Ill. }
$$




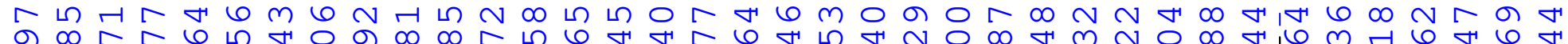
б,

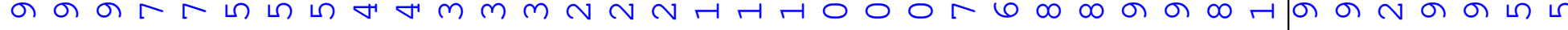

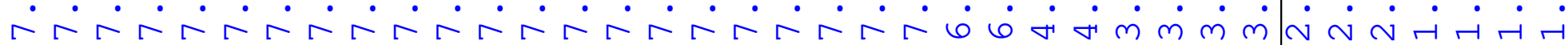

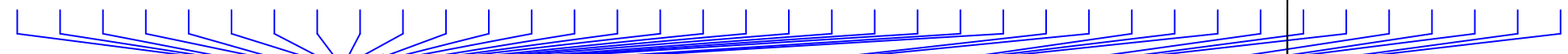
2

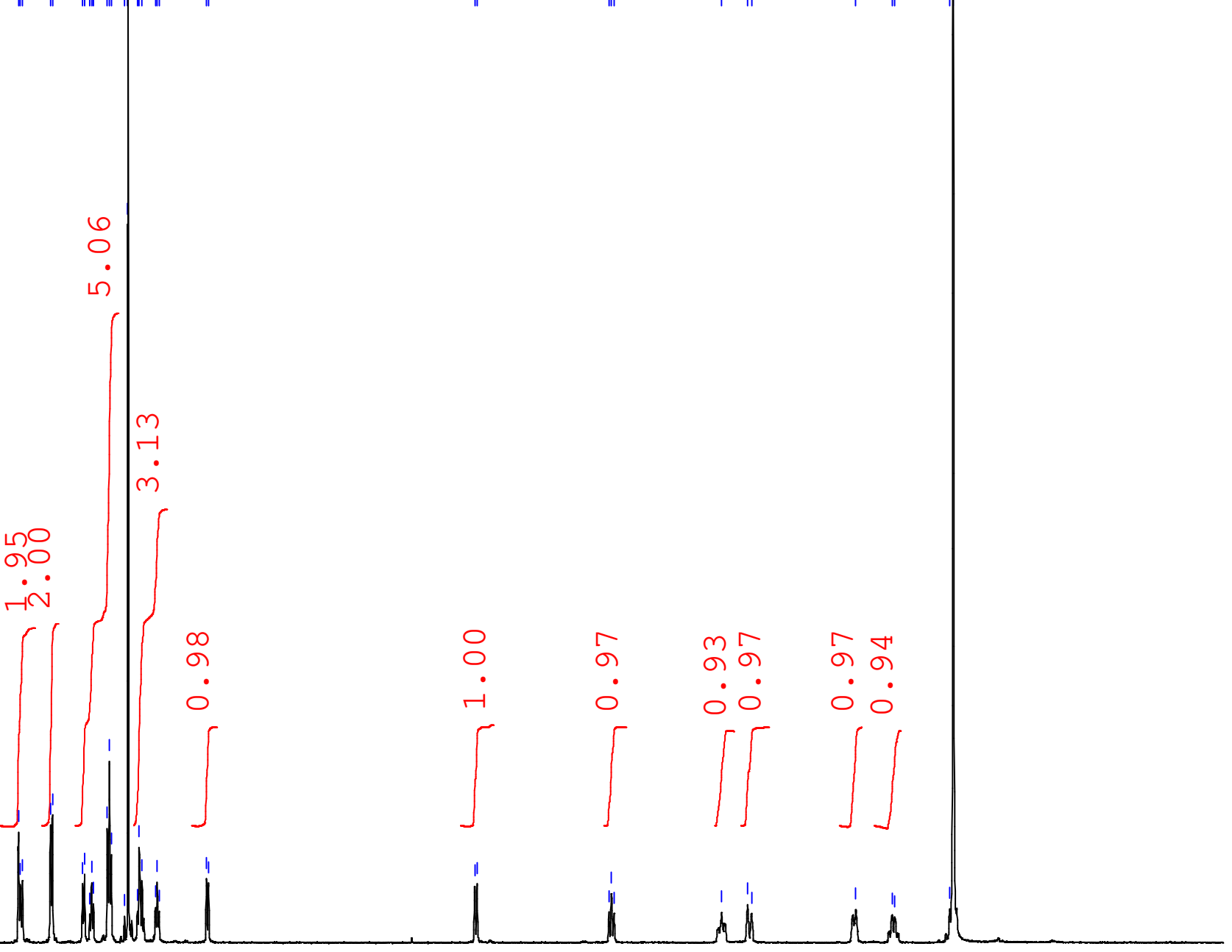



${ }^{1} \mathrm{H}$ NMR $\left(600 \mathrm{MHz}, \mathrm{CDCl}_{3}\right)$ 

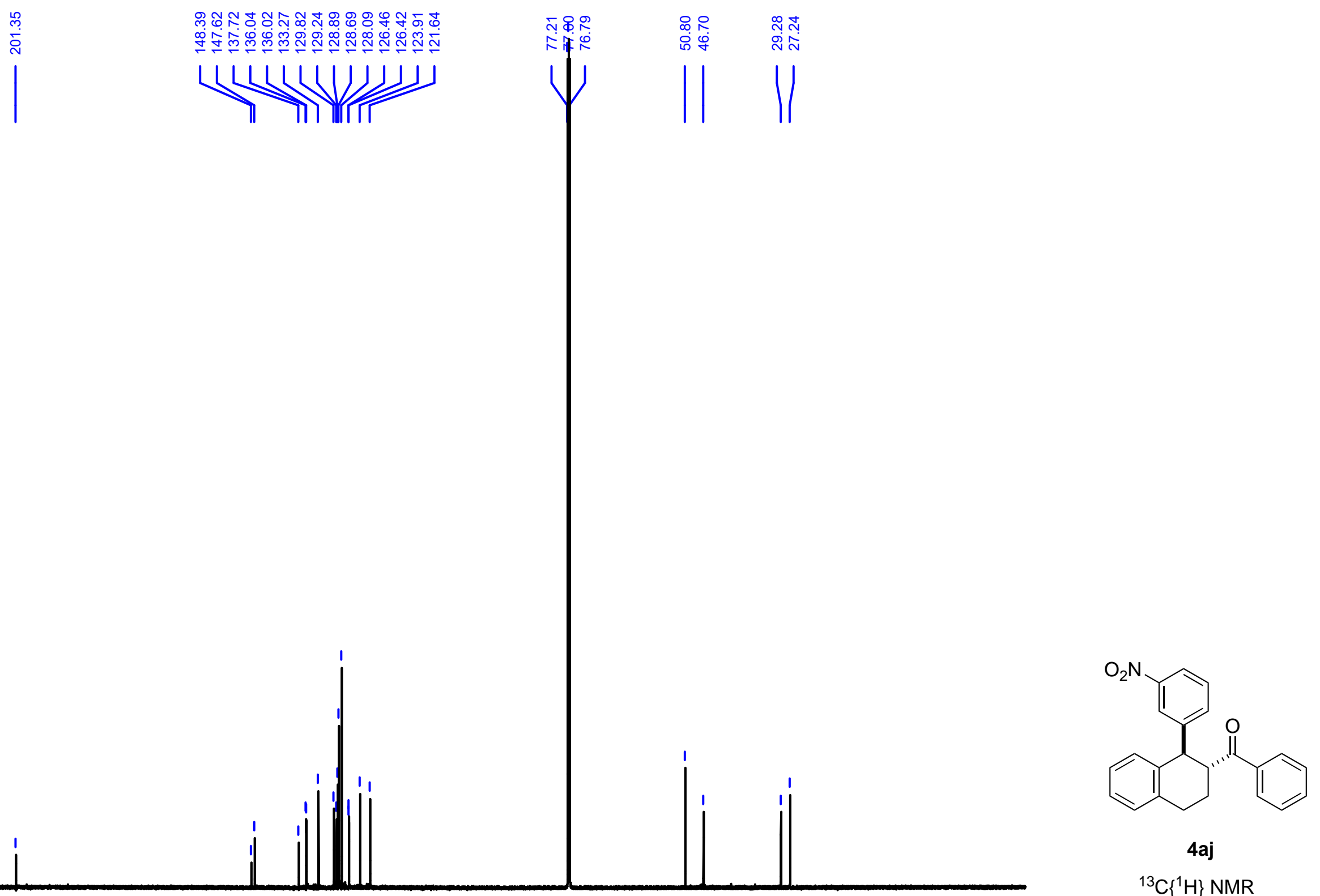

4aj

${ }^{13} \mathrm{C}\left\{{ }^{1} \mathrm{H}\right\}$ NMR

$\left(150 \mathrm{MHz}, \mathrm{CDCl}_{3}\right.$ )

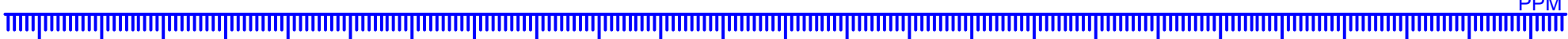

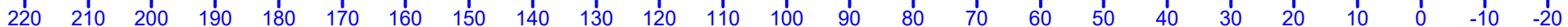


सmon G

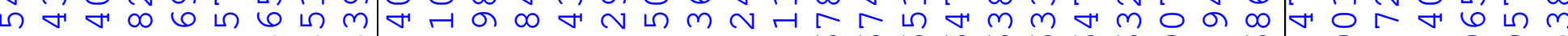

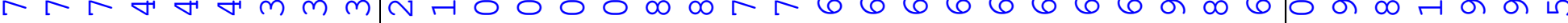

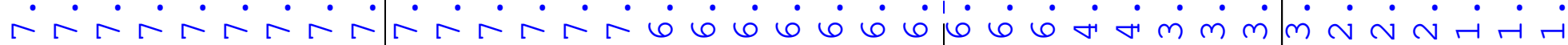

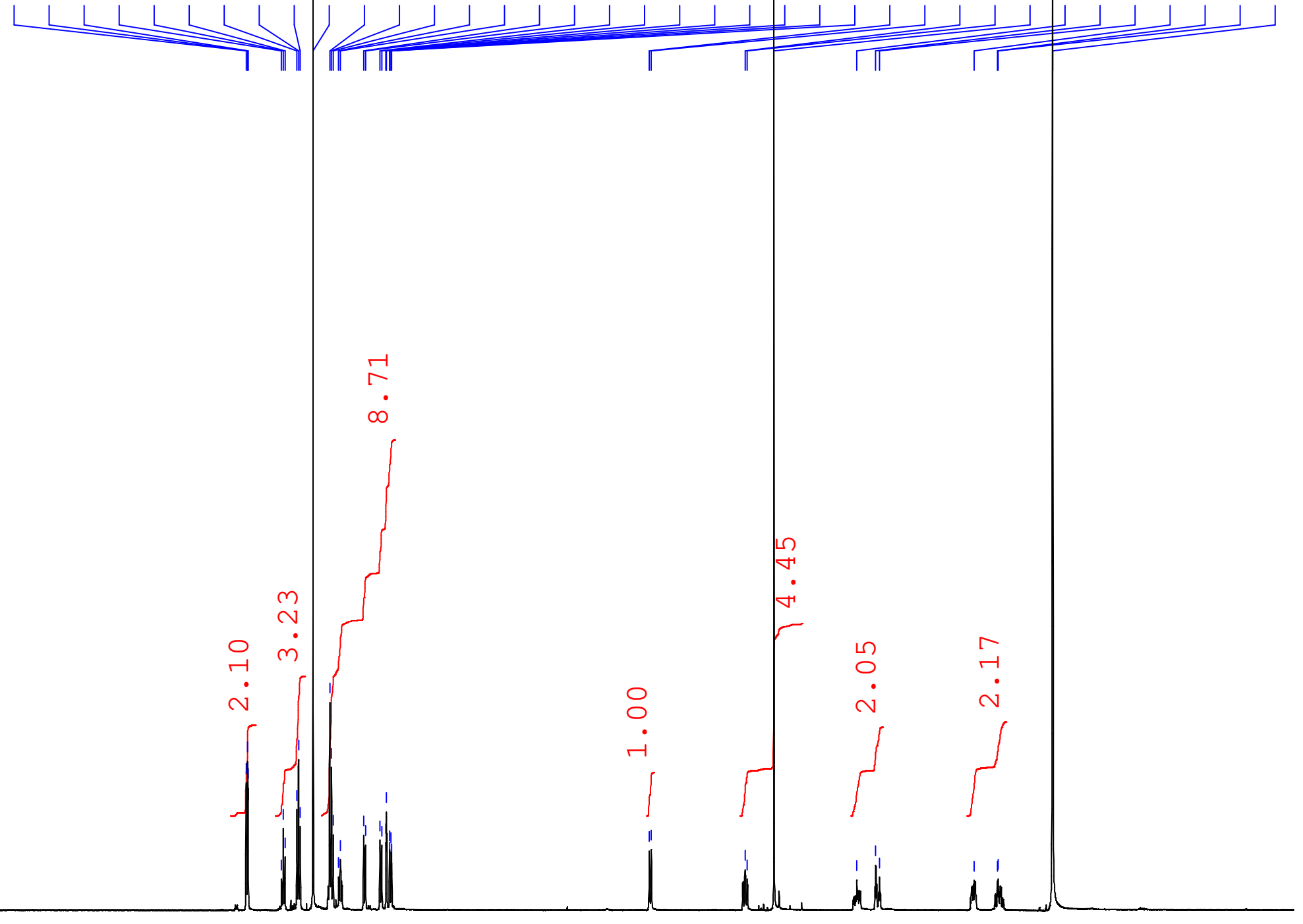

PPM

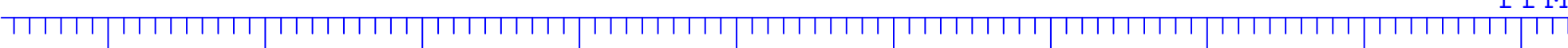

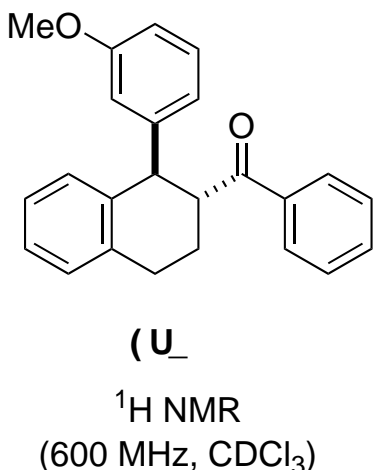




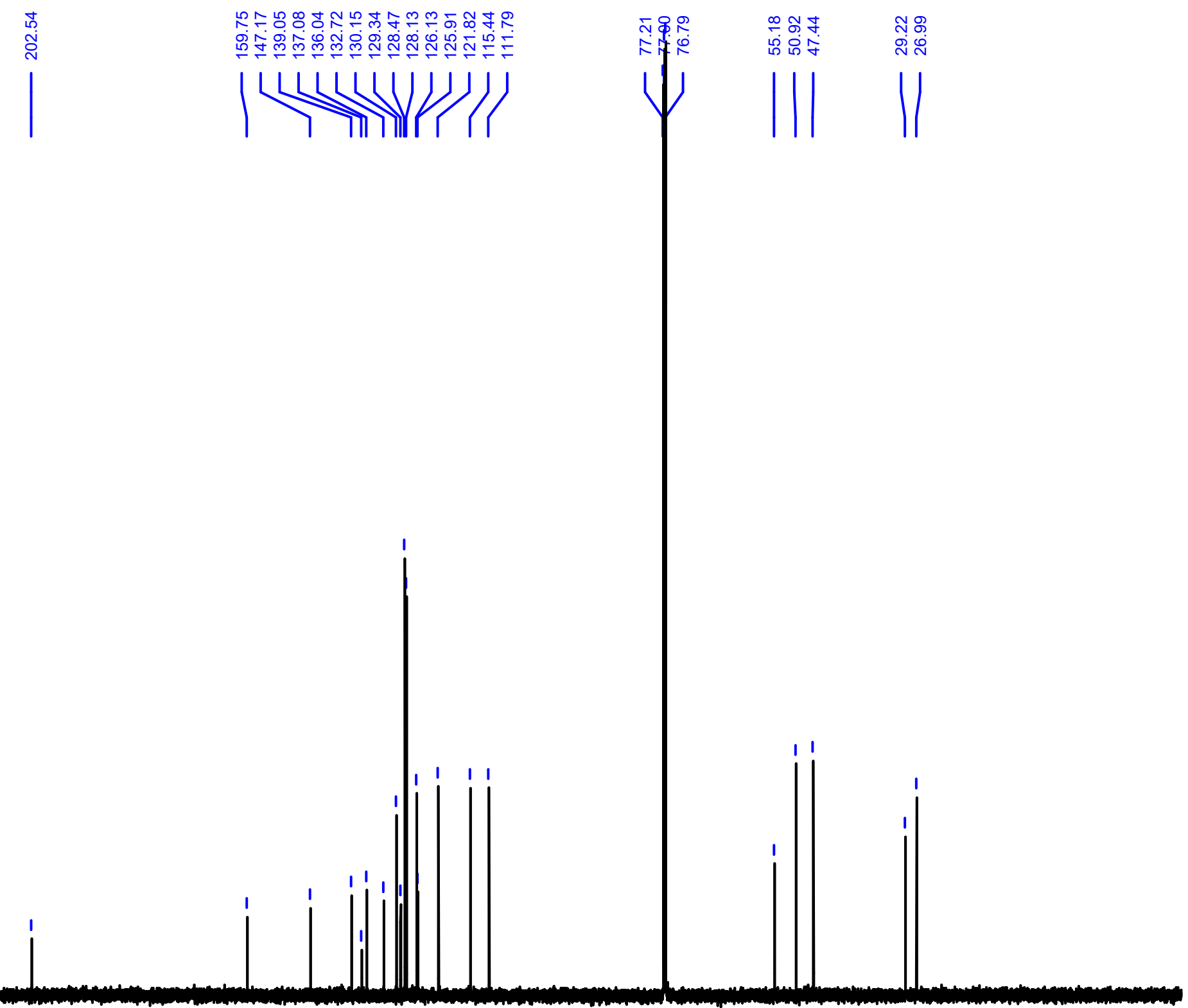

TाIा|

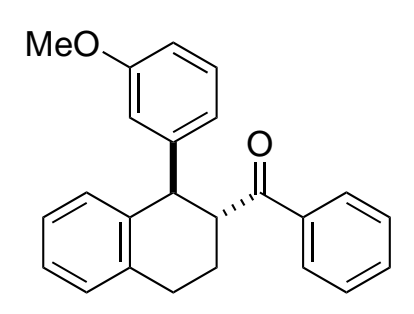

4ak

${ }^{13} \mathrm{C}\left\{{ }^{1} \mathrm{H}\right\}$ NMR

$\left(150 \mathrm{MHz}, \mathrm{CDCl}_{3}\right.$ ) 
n ఎ

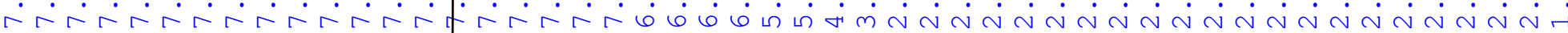
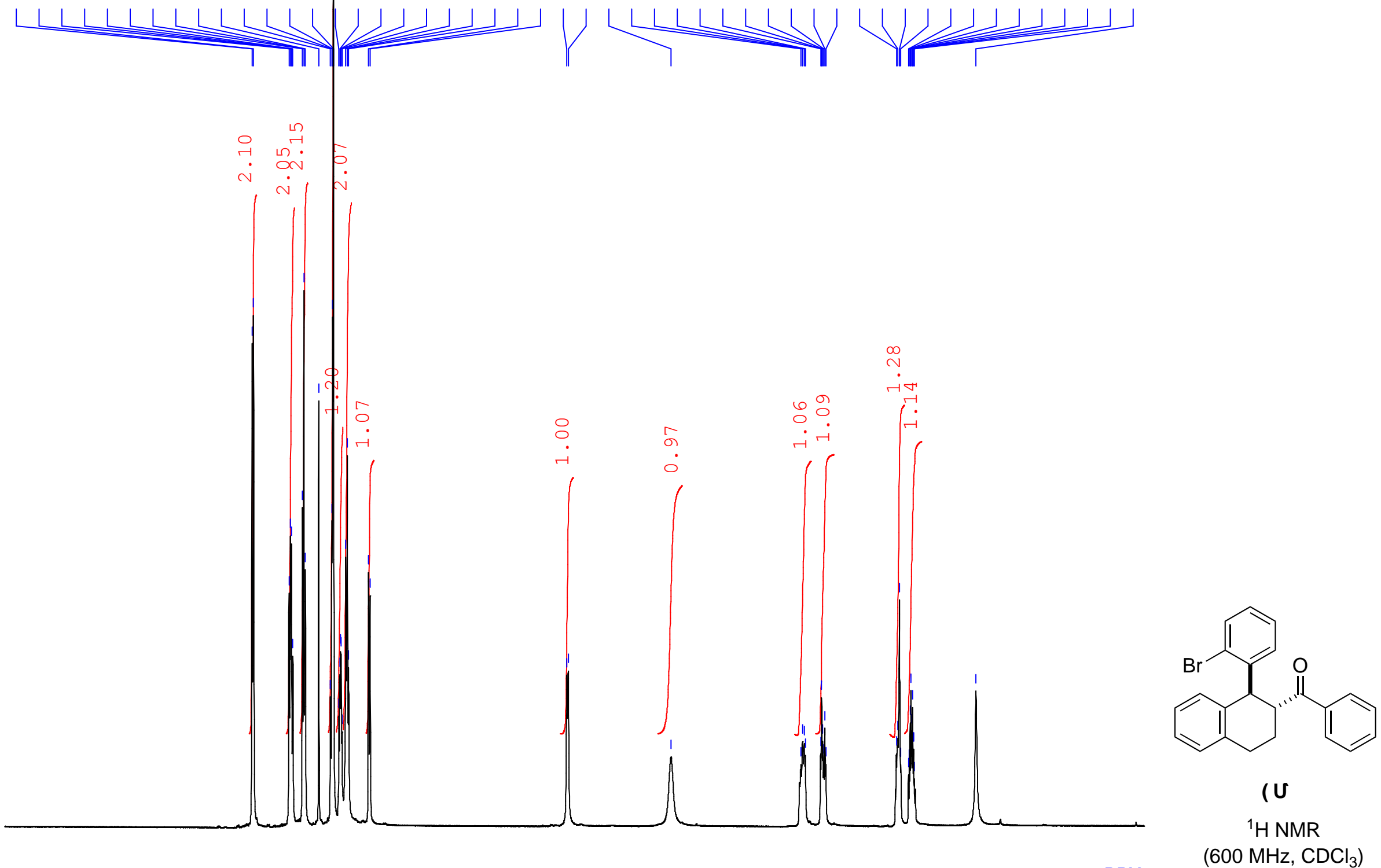


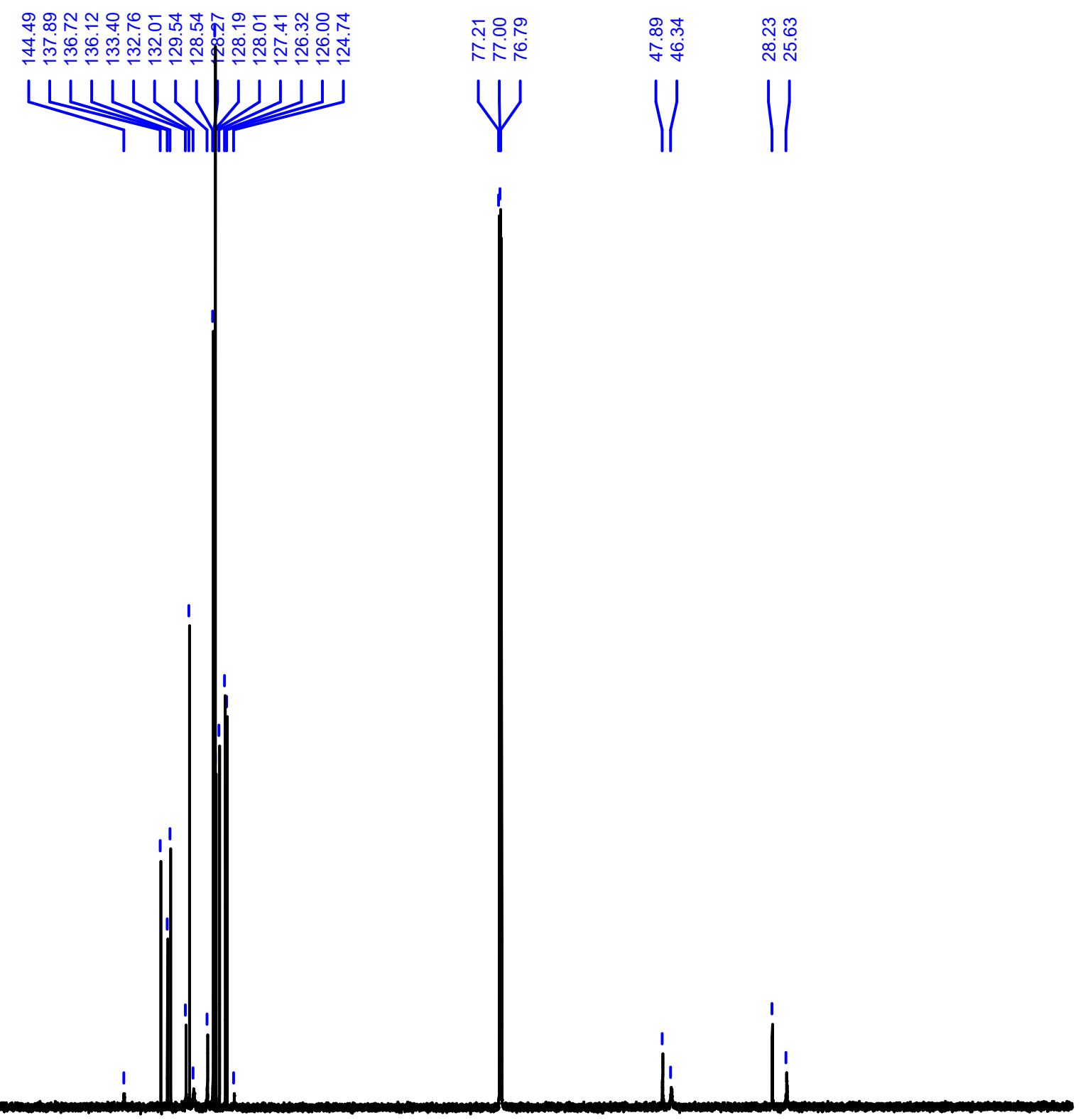

Tाmा

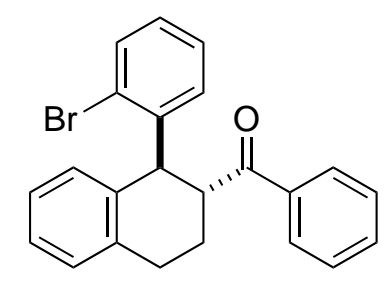

4al

${ }^{13} \mathrm{C}\left\{{ }^{1} \mathrm{H}\right\} \mathrm{NMR}$ $\left(150 \mathrm{MHz}, \mathrm{CDCl}_{3}\right)$ at $50^{\circ} \mathrm{C}$ 


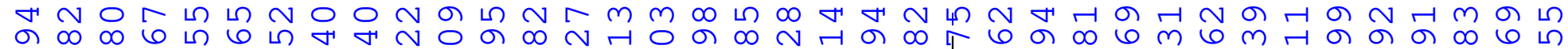

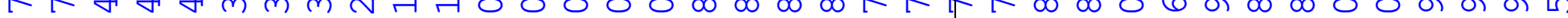

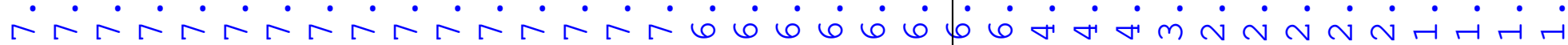

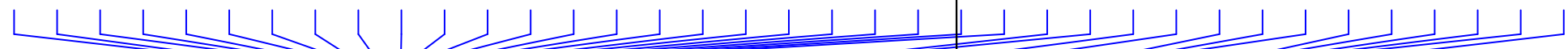
n \|\|\|\|$\|$
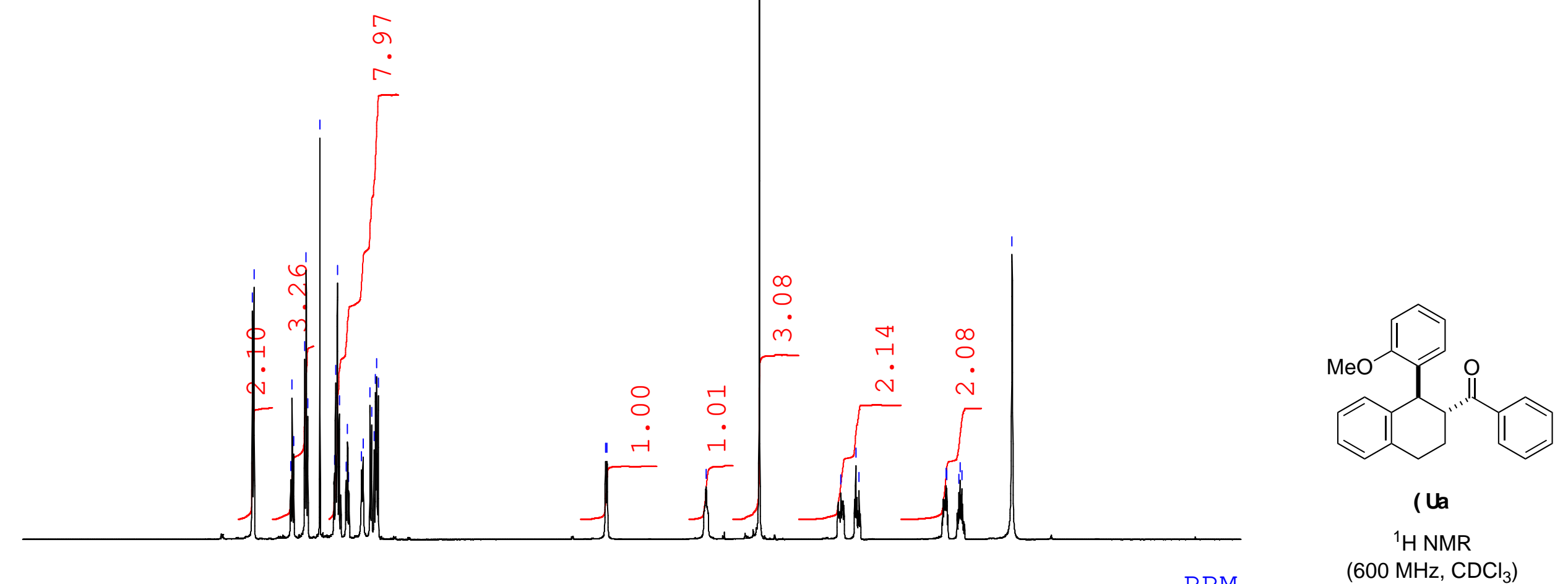


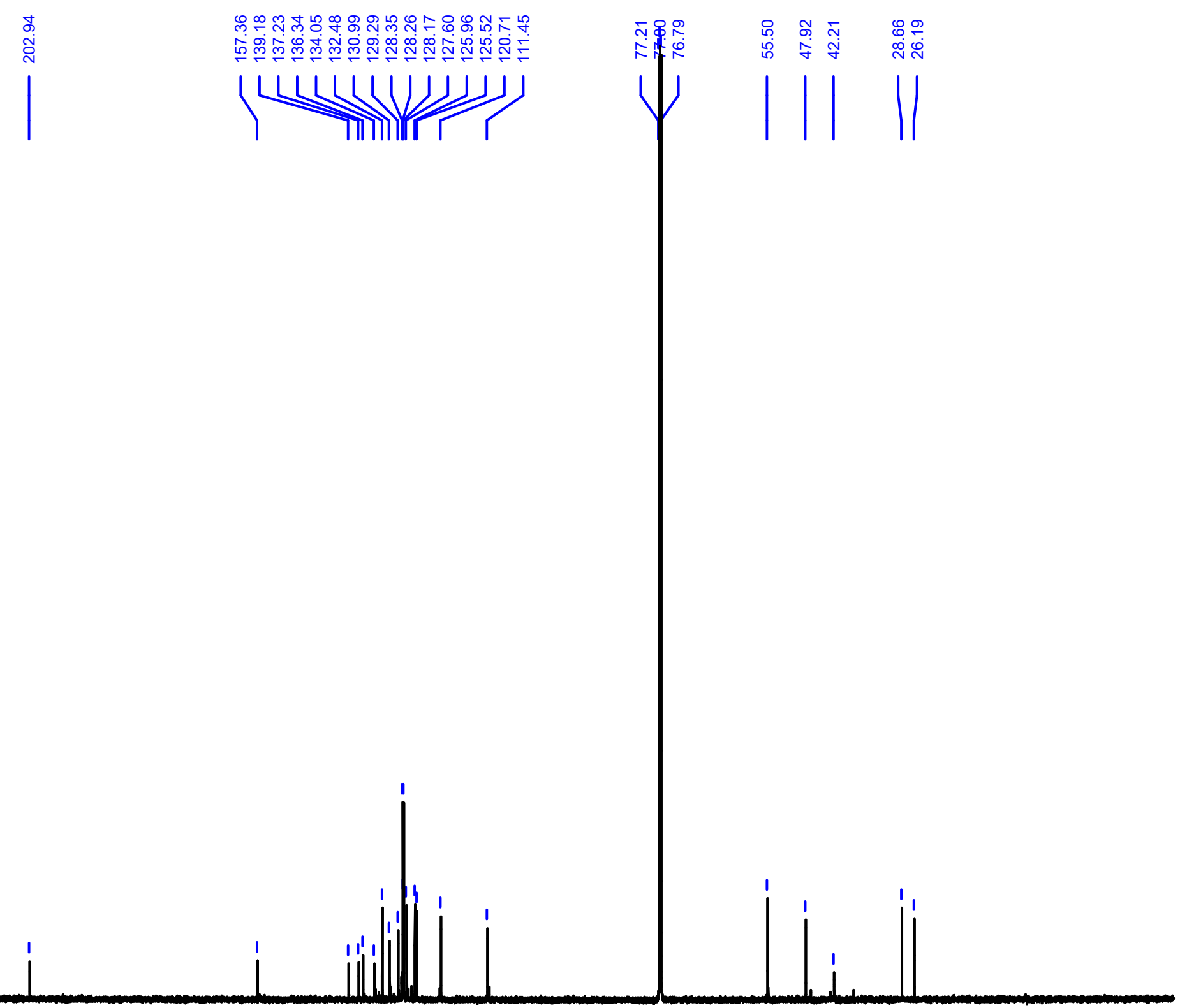

TाIा

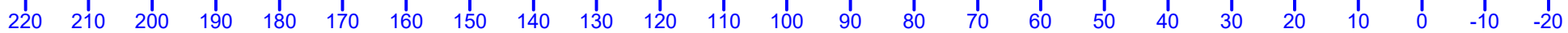

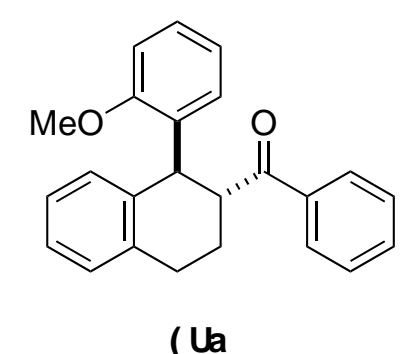

${ }^{13} \mathrm{C}\left\{{ }^{1} \mathrm{H}\right\} \mathrm{NMR}$

$\left(150 \mathrm{MHz}, \mathrm{CDCl}_{3}\right)$

at $50{ }^{\circ} \mathrm{C}$ 


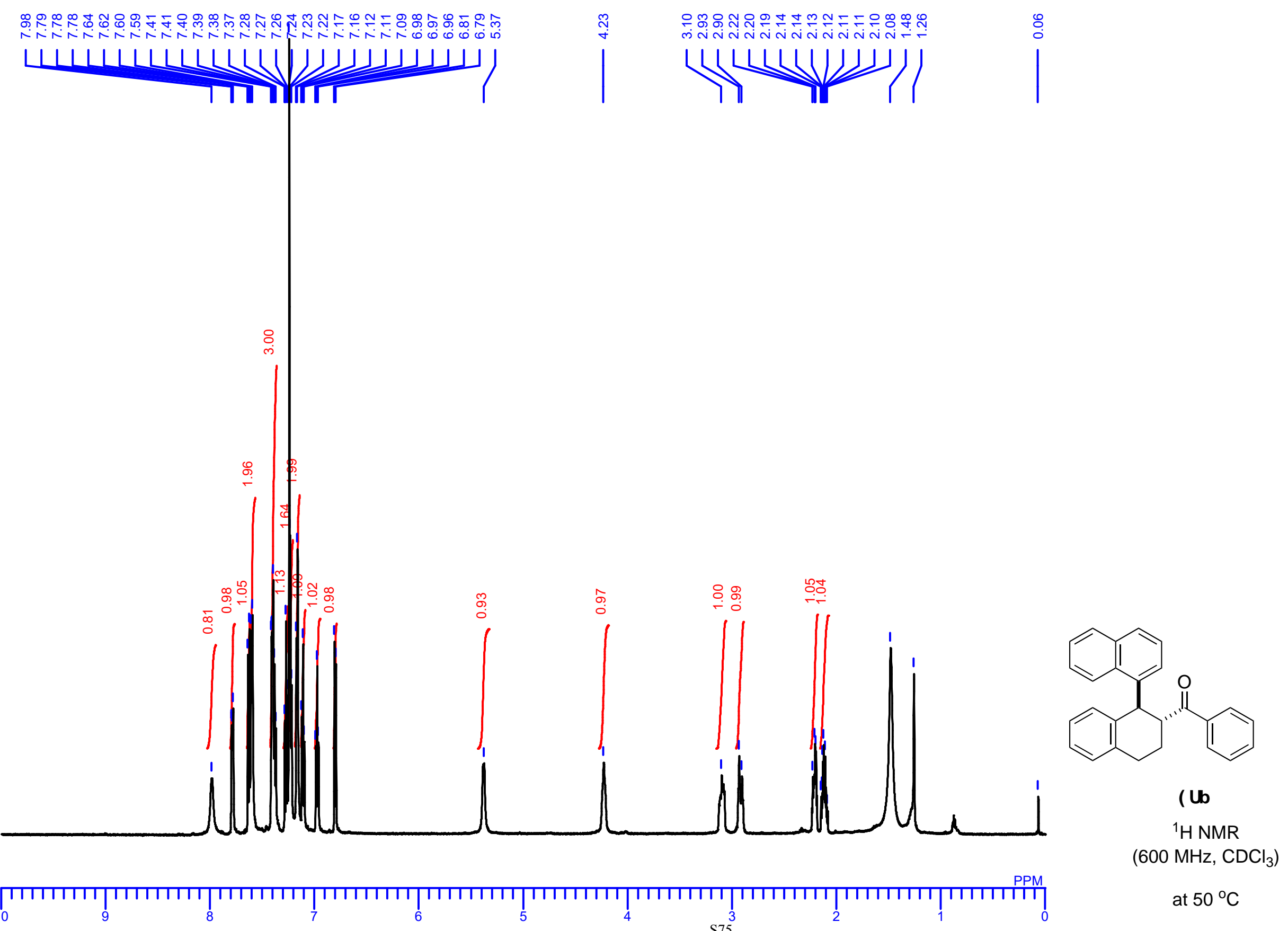




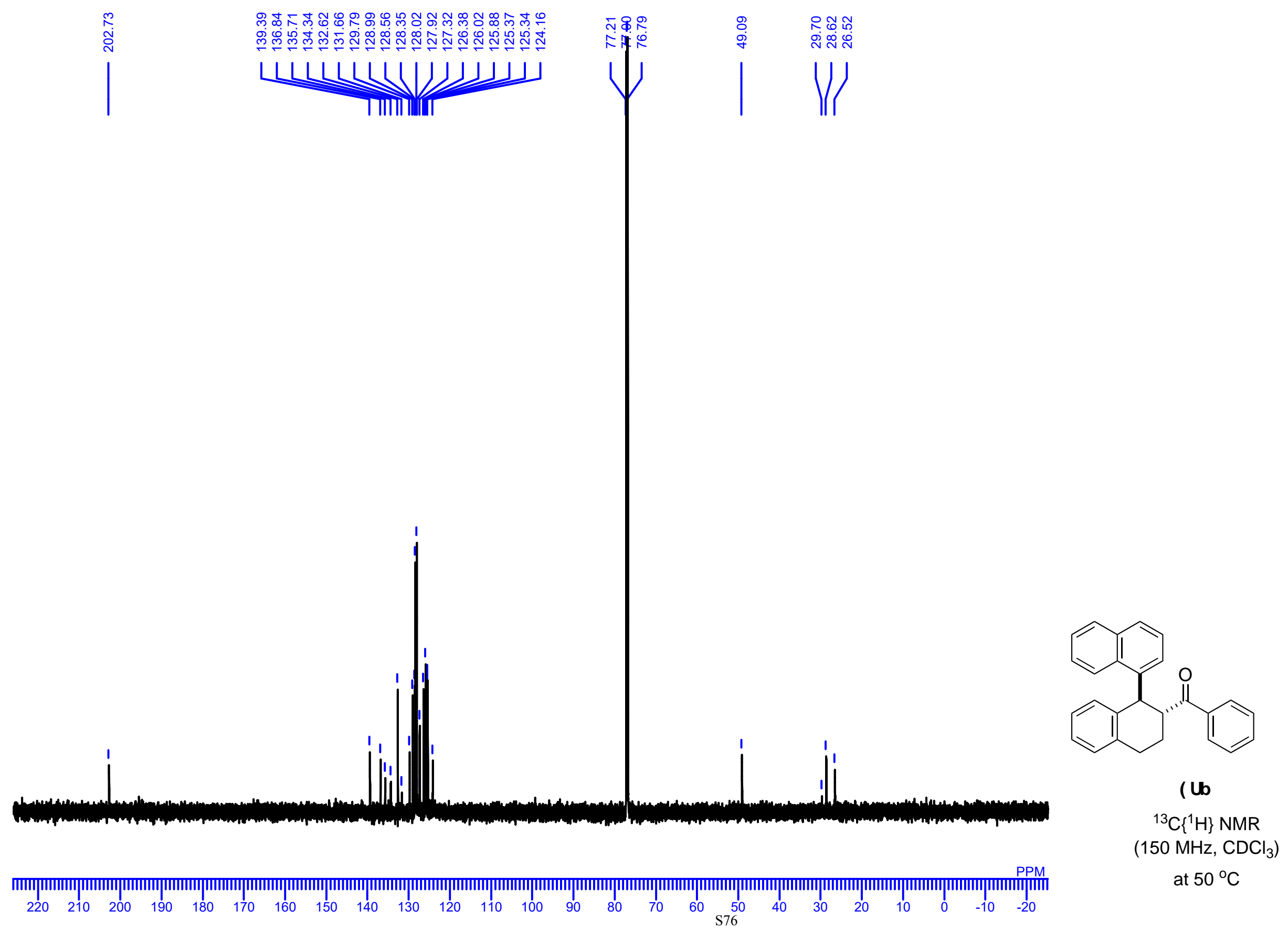




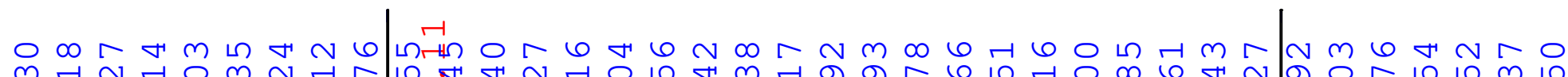

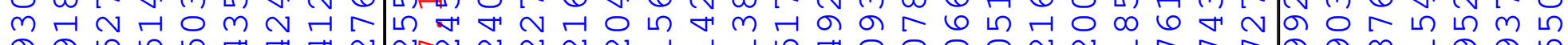

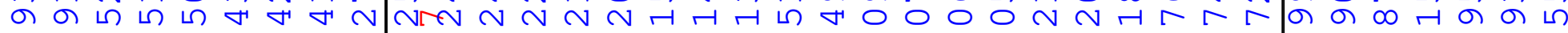

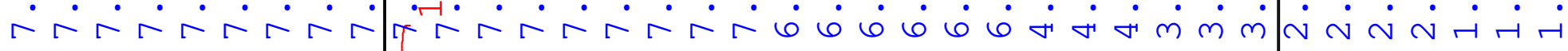

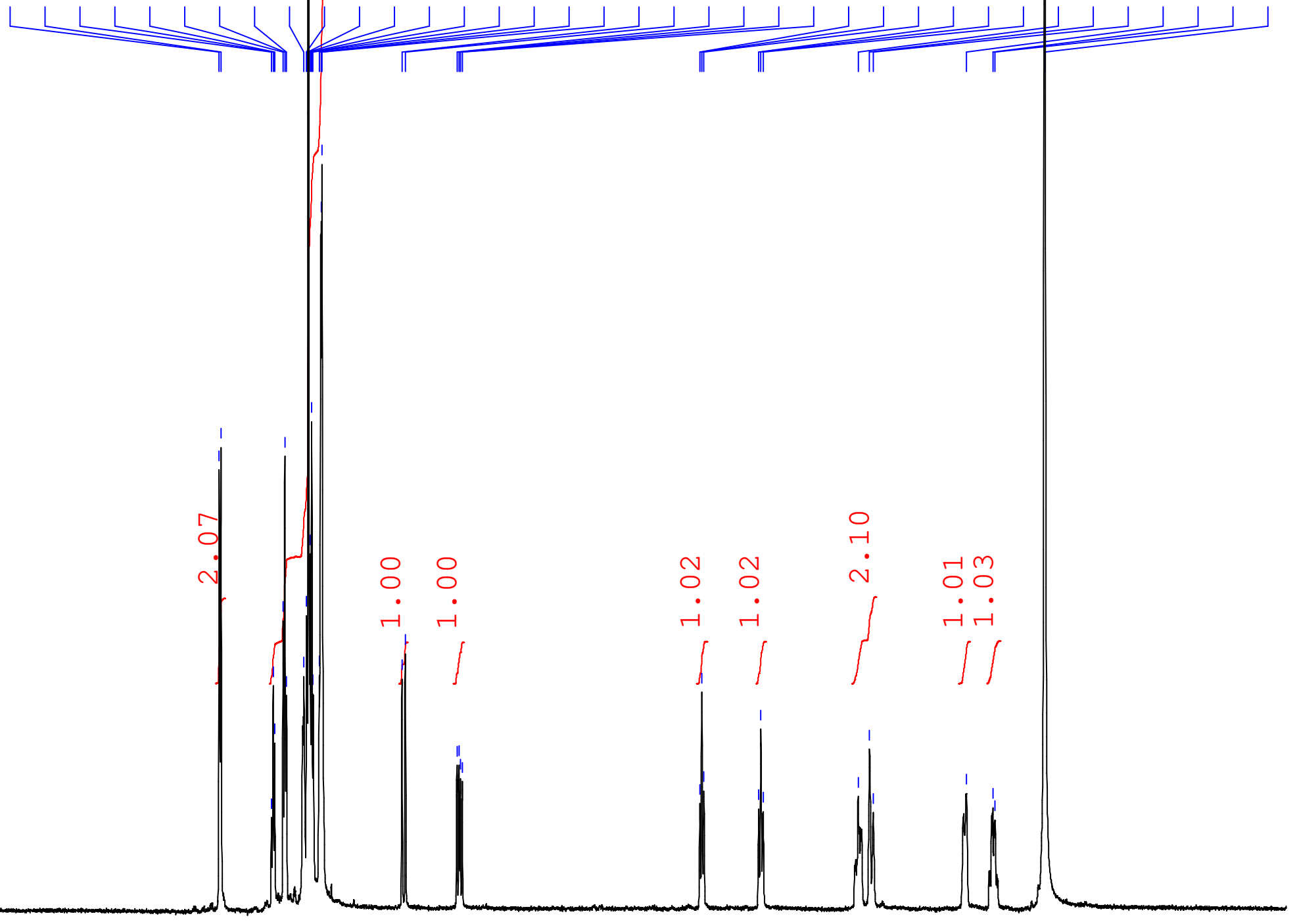

PPM



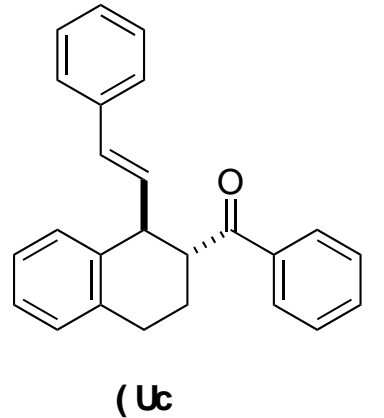

${ }^{1} \mathrm{H}$ NMR $\left(600 \mathrm{MHz}, \mathrm{CDCl}_{3}\right)$ 


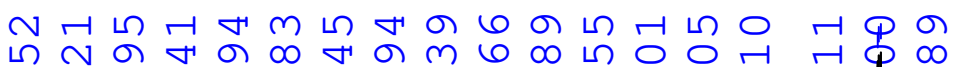



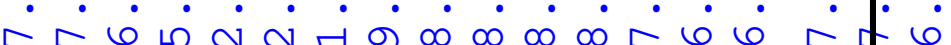

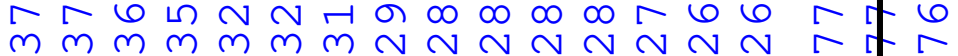

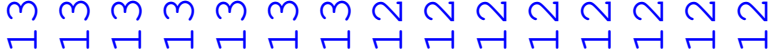
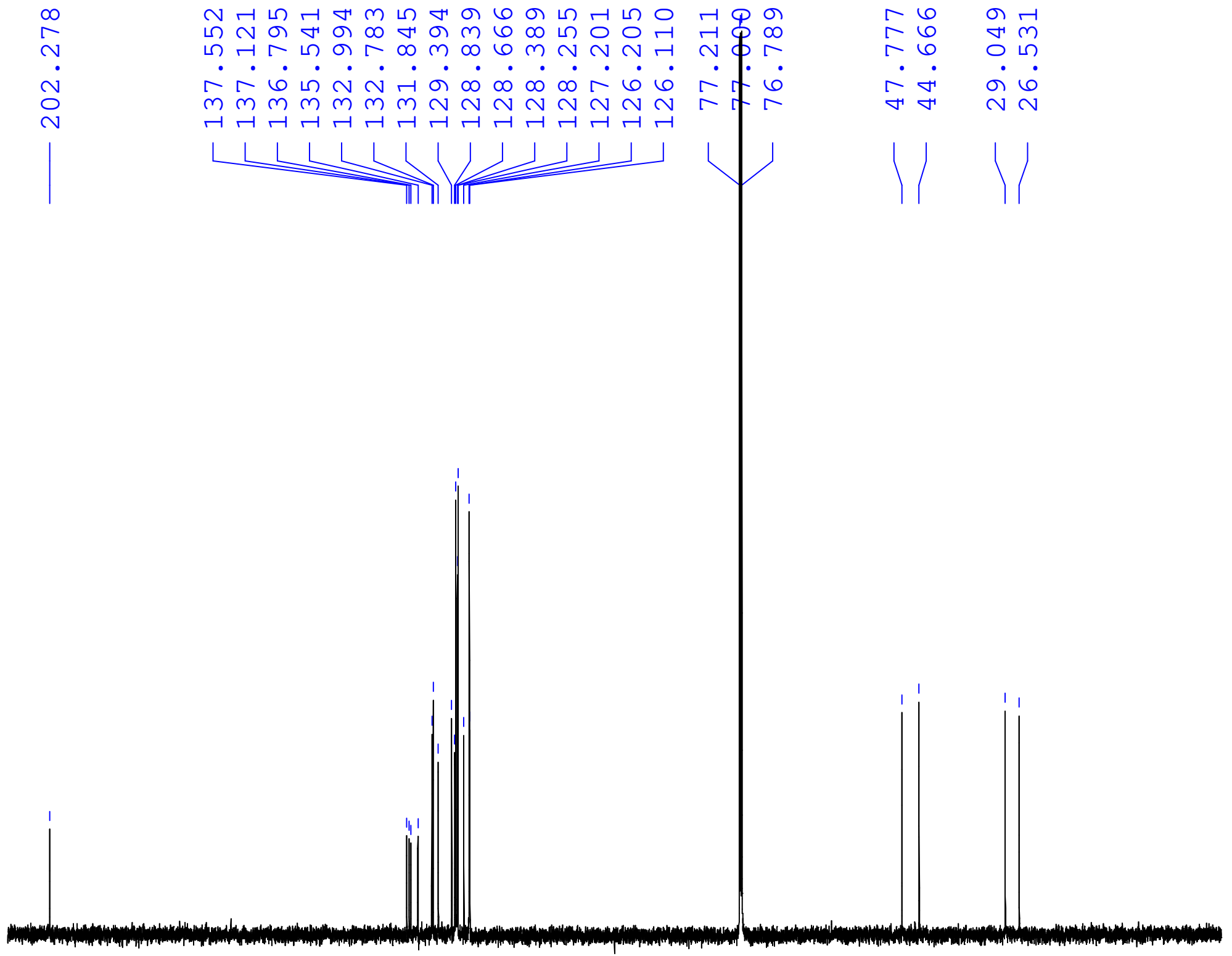

PPM

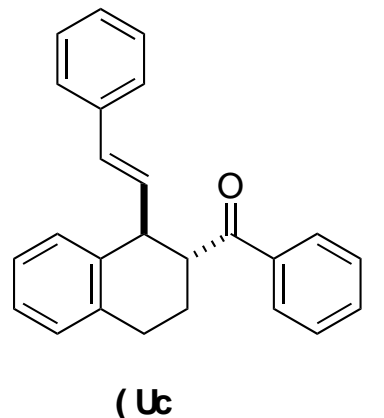

${ }^{3} \mathrm{C}\left\{{ }^{1} \mathrm{H}\right\} \mathrm{NMR}$ $\left(150 \mathrm{MHz}, \mathrm{CDCl}_{3}\right)$ 


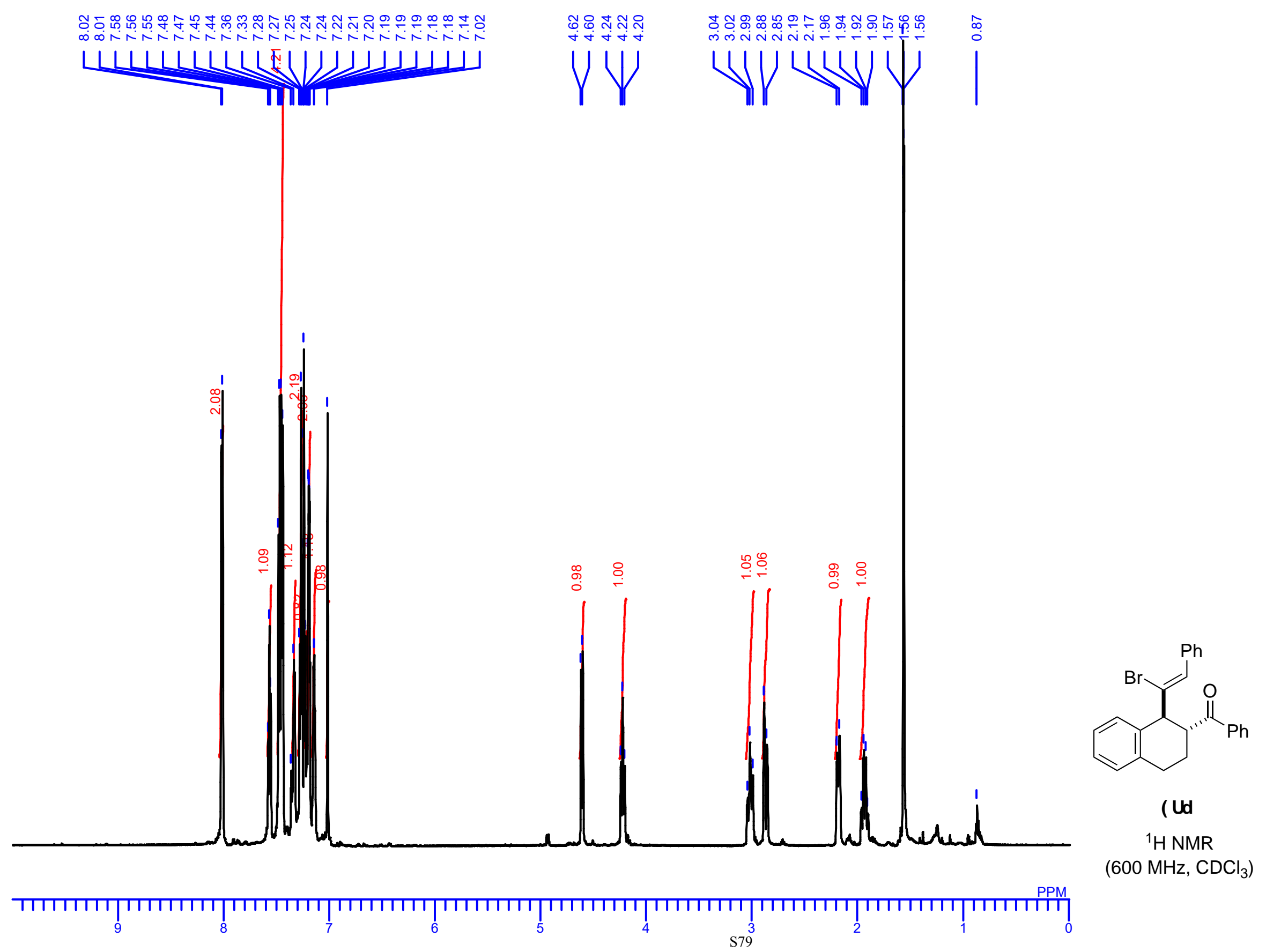




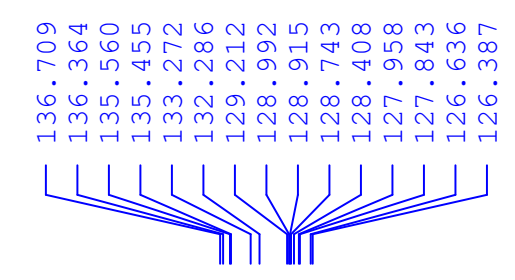

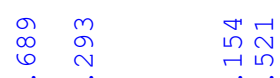

i்

III

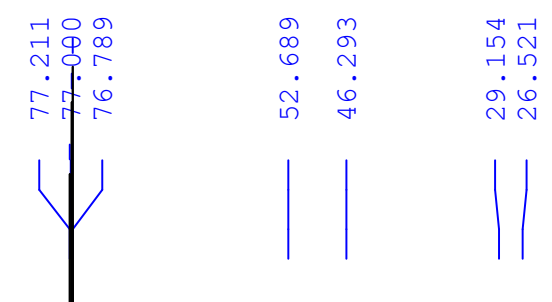

$\mathrm{Ph}$

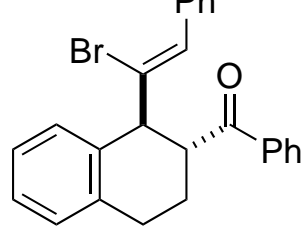

${ }^{13} \mathrm{C}\left\{{ }^{1} \mathrm{H}\right\}$ NMR $\left(150 \mathrm{MHz}, \mathrm{CDCl}_{3}\right)$

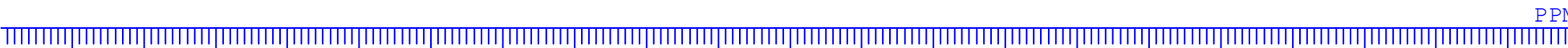

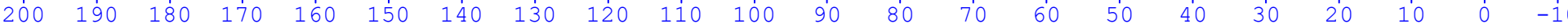




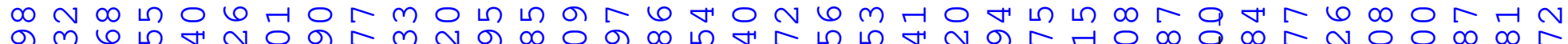

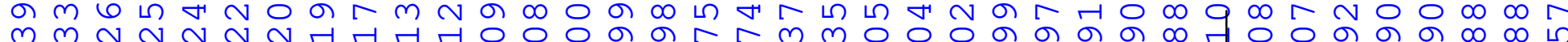

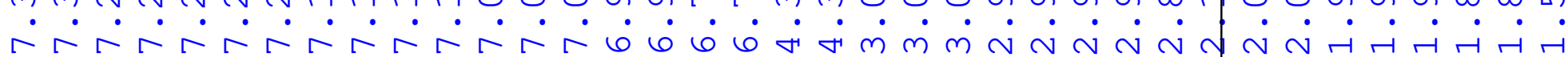

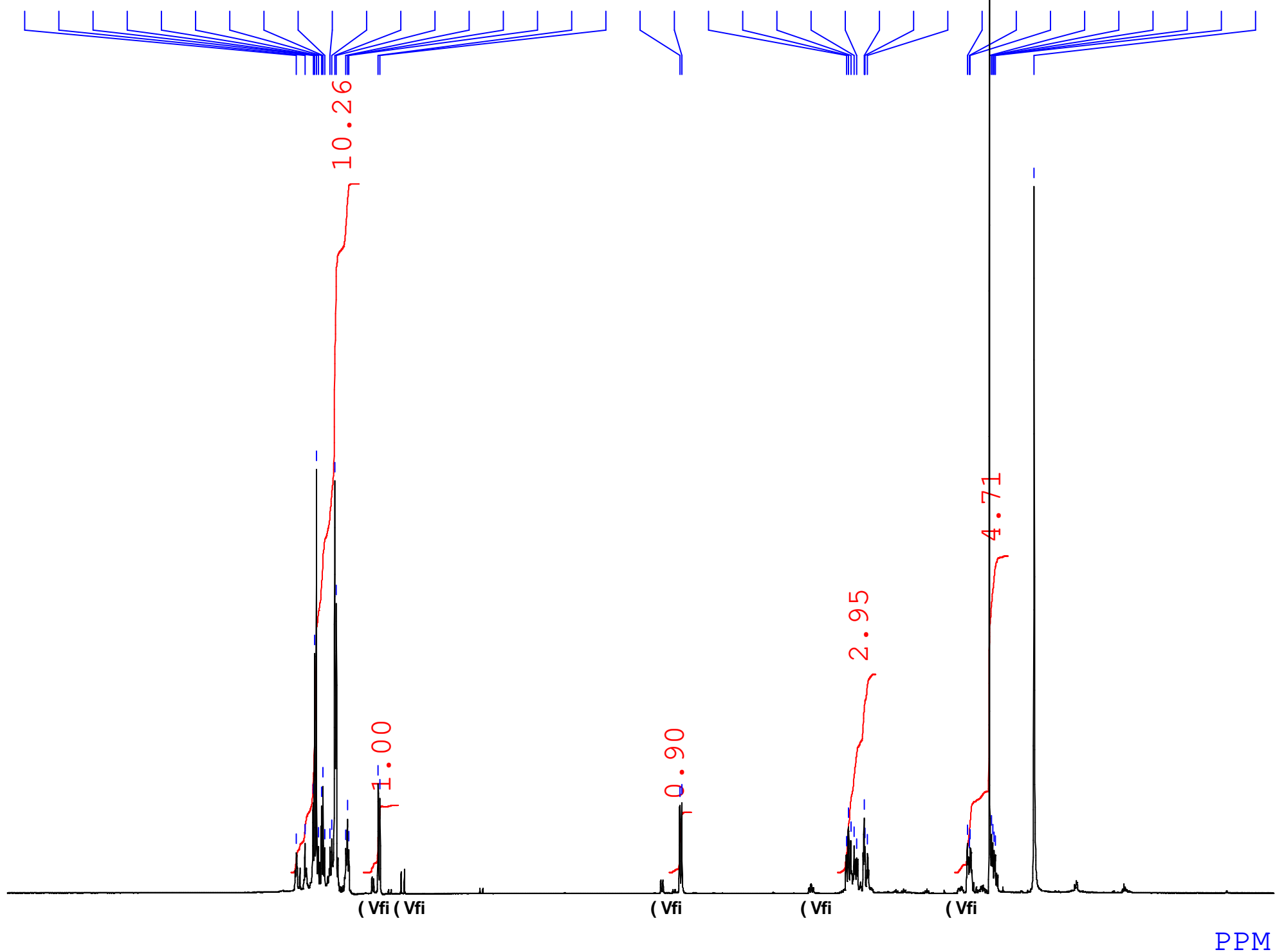

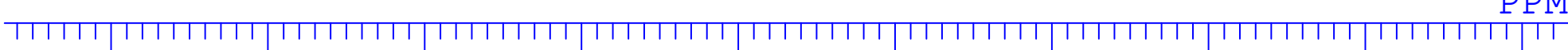

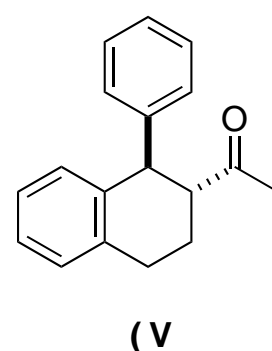

${ }^{1} \mathrm{H}$ NMR

(600 MHz, $\mathrm{CDCl}_{3}$ ) 

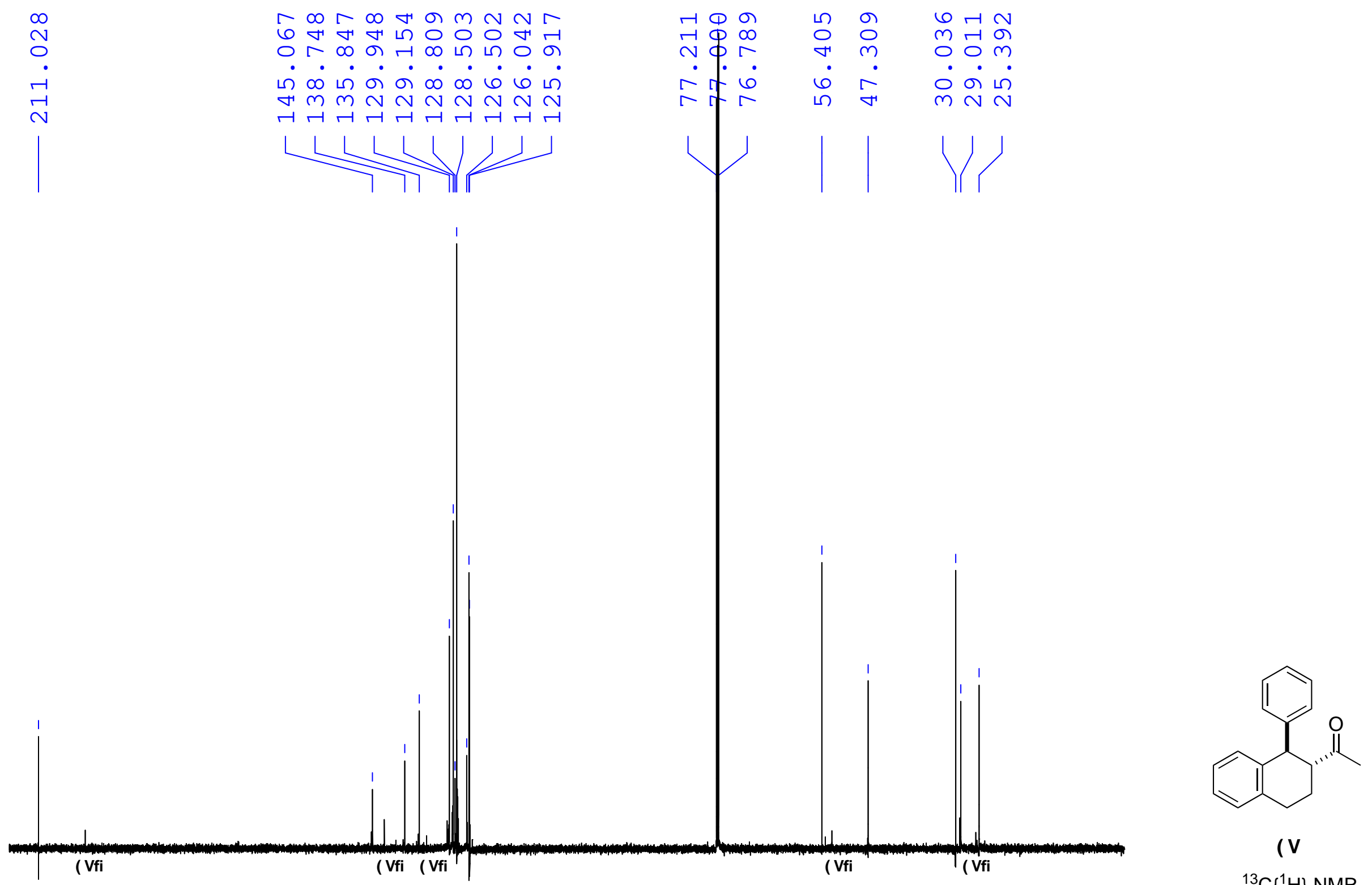

${ }^{13} \mathrm{C}\left\{{ }^{1} \mathrm{H}\right\}$ NMR

PPM

$\left(150 \mathrm{MHz}, \mathrm{CDCl}_{3}\right.$ ) 


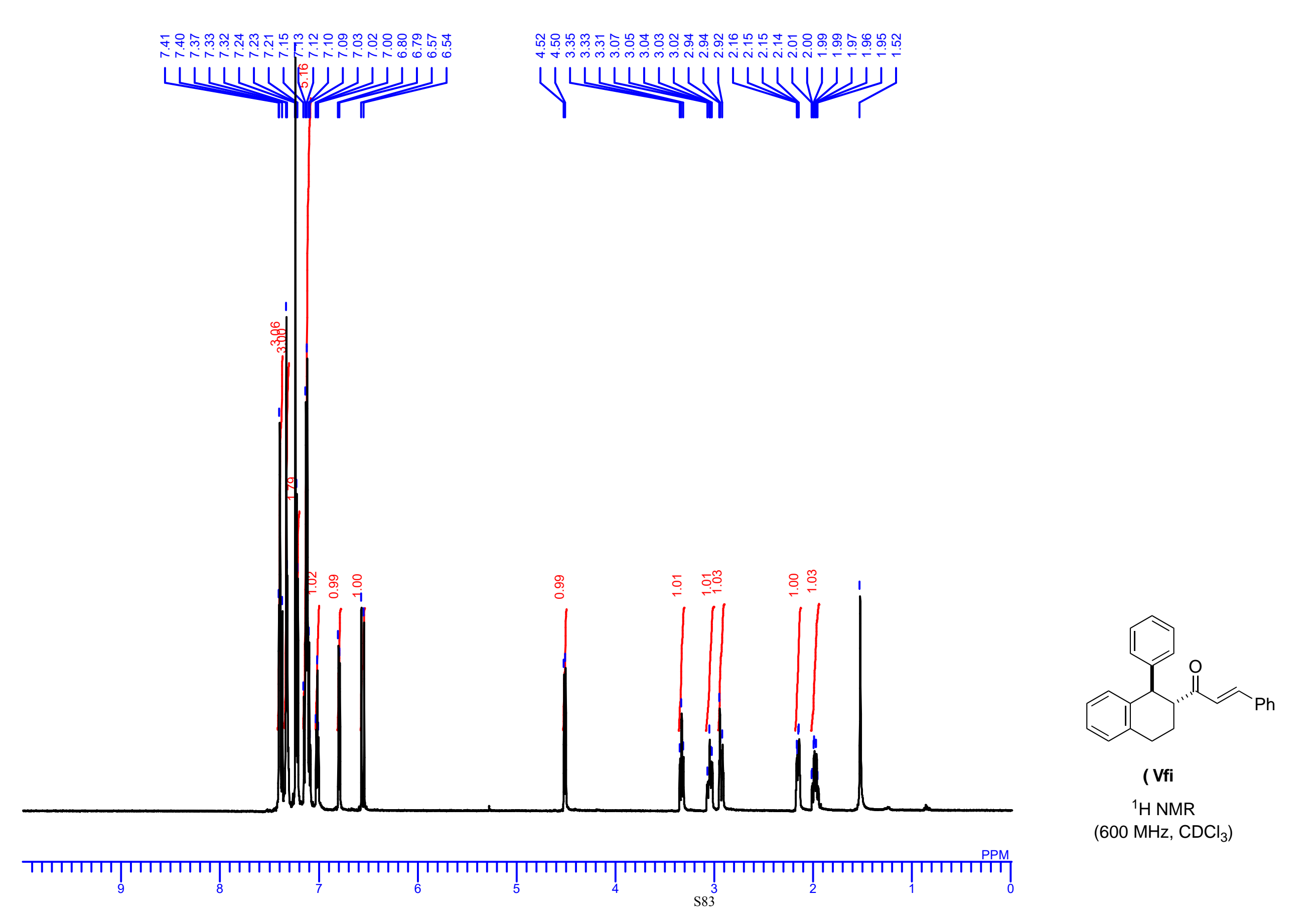




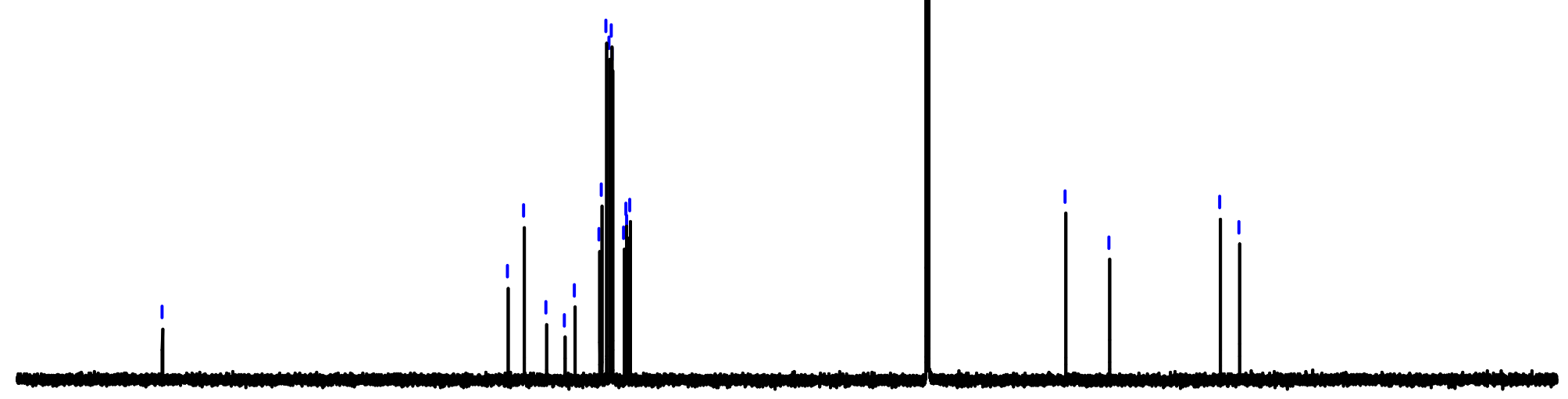

TाIा

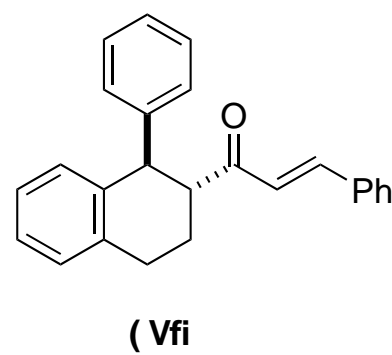

${ }^{13} \mathrm{C}\left\{{ }^{1} \mathrm{H}\right\}$ NMR $\left(150 \mathrm{MHz}, \mathrm{CDCl}_{3}\right.$ ) 

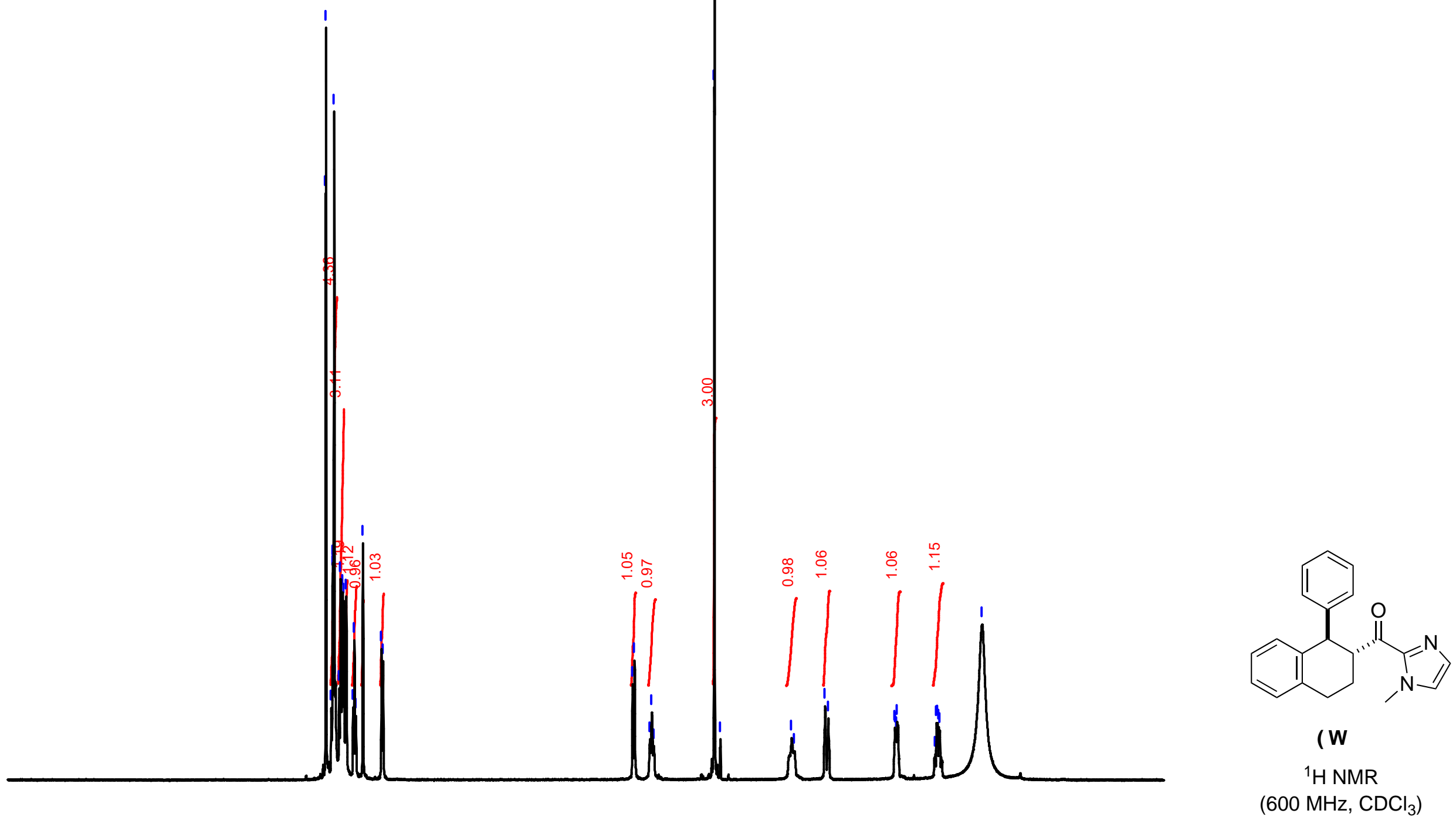


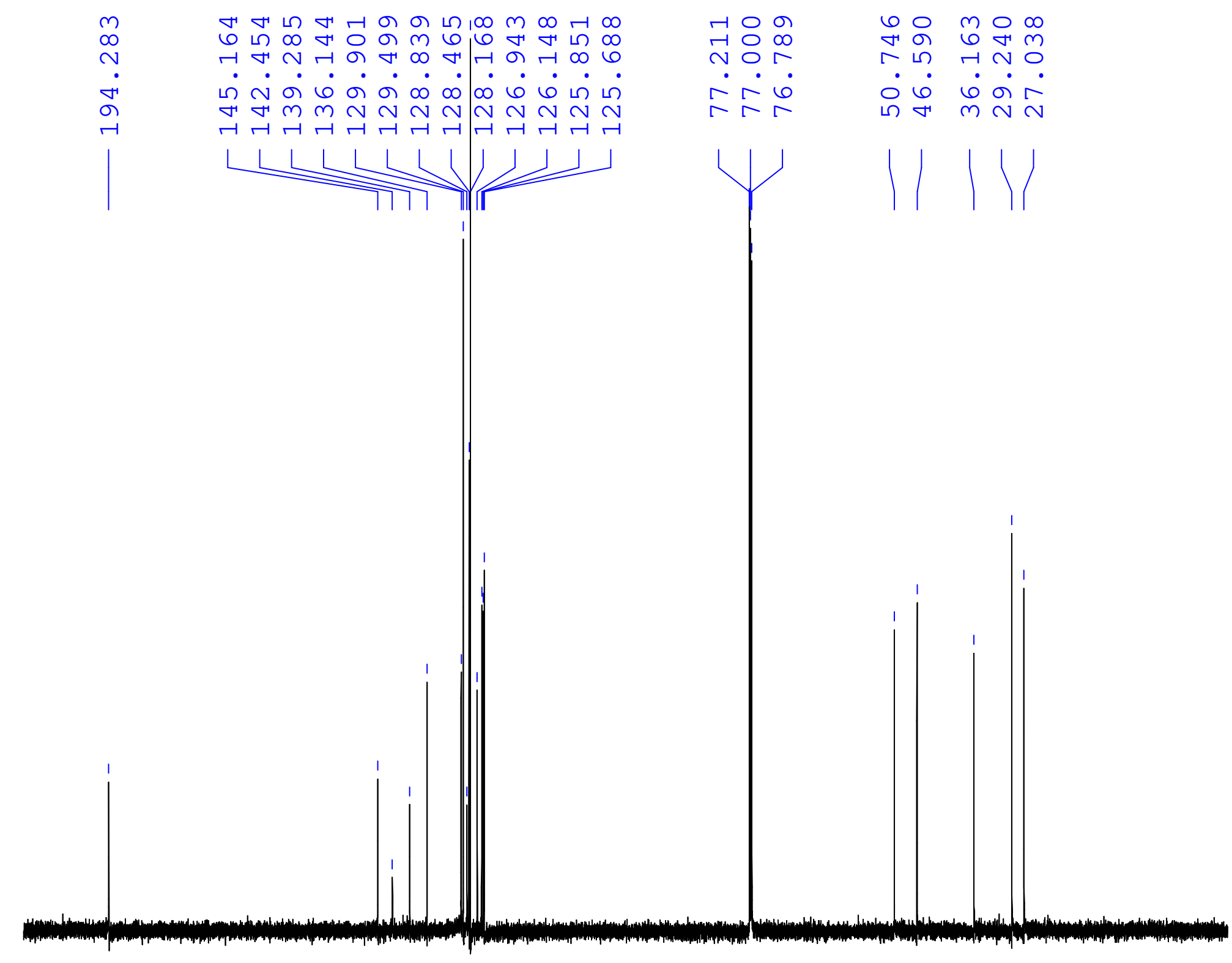

PPM

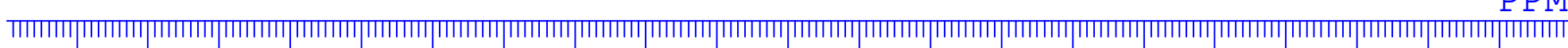

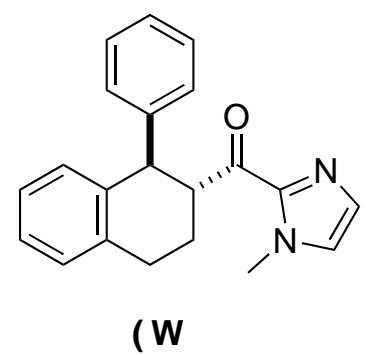

${ }^{13} \mathrm{C}\left\{{ }^{1} \mathrm{H}\right\} \mathrm{NMR}$ $\left(150 \mathrm{MHz}, \mathrm{CDCl}_{3}\right)$ 


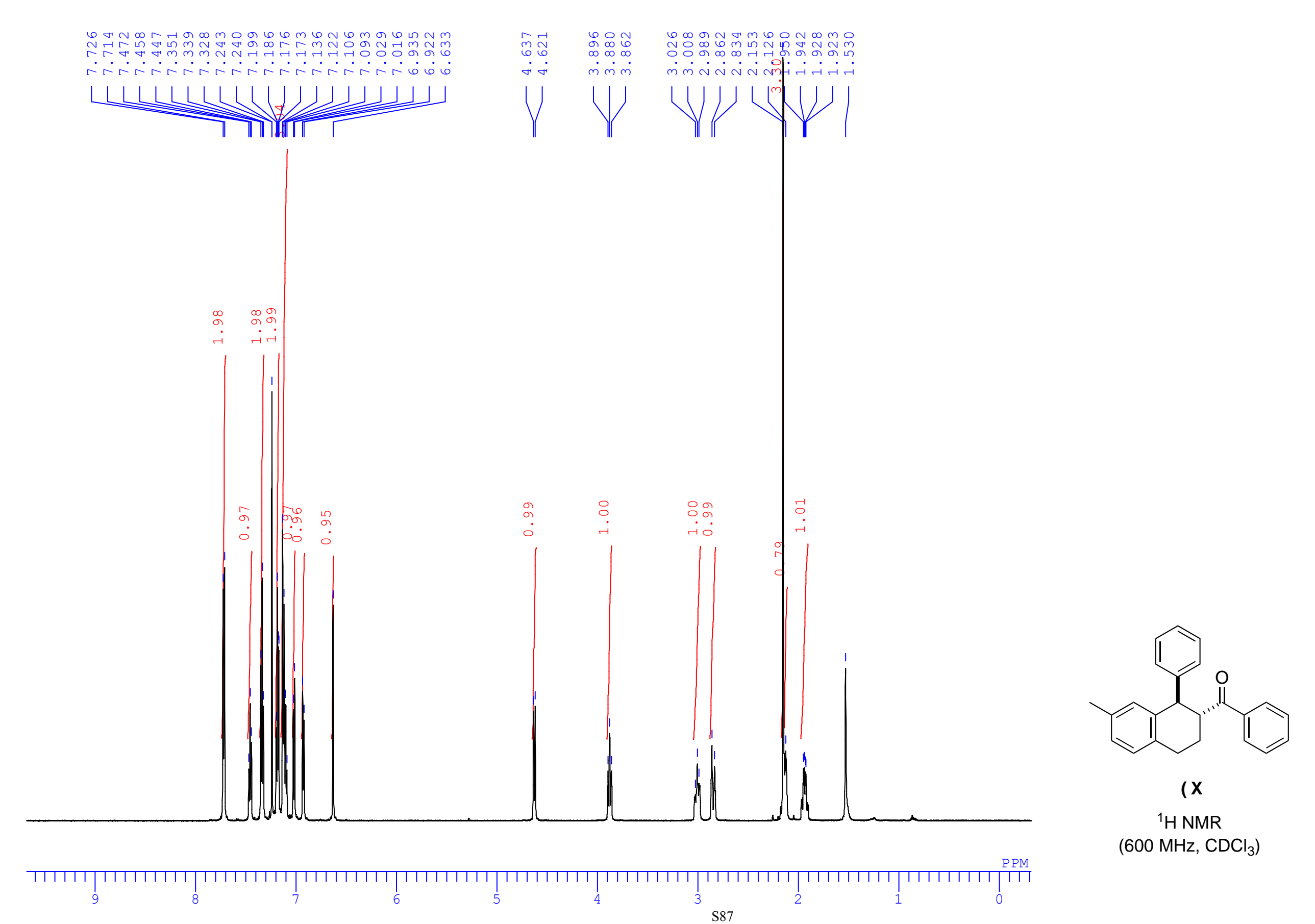



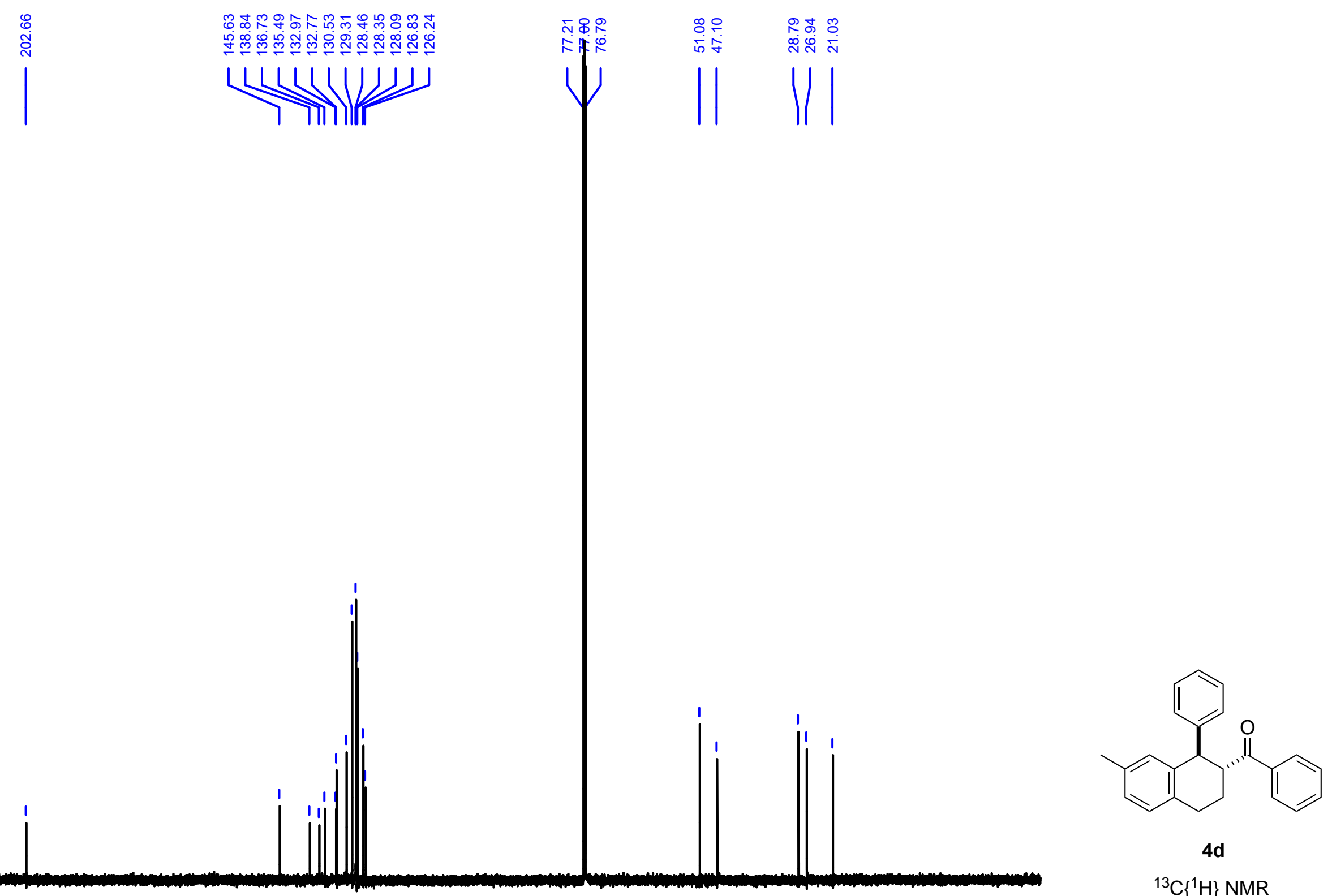

${ }^{13} \mathrm{C}\left\{{ }^{1} \mathrm{H}\right\}$ NMR $\left(150 \mathrm{MHz}, \mathrm{CDCl}_{3}\right)$

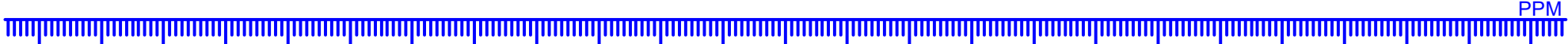

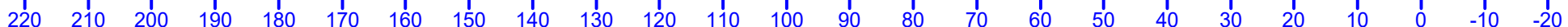




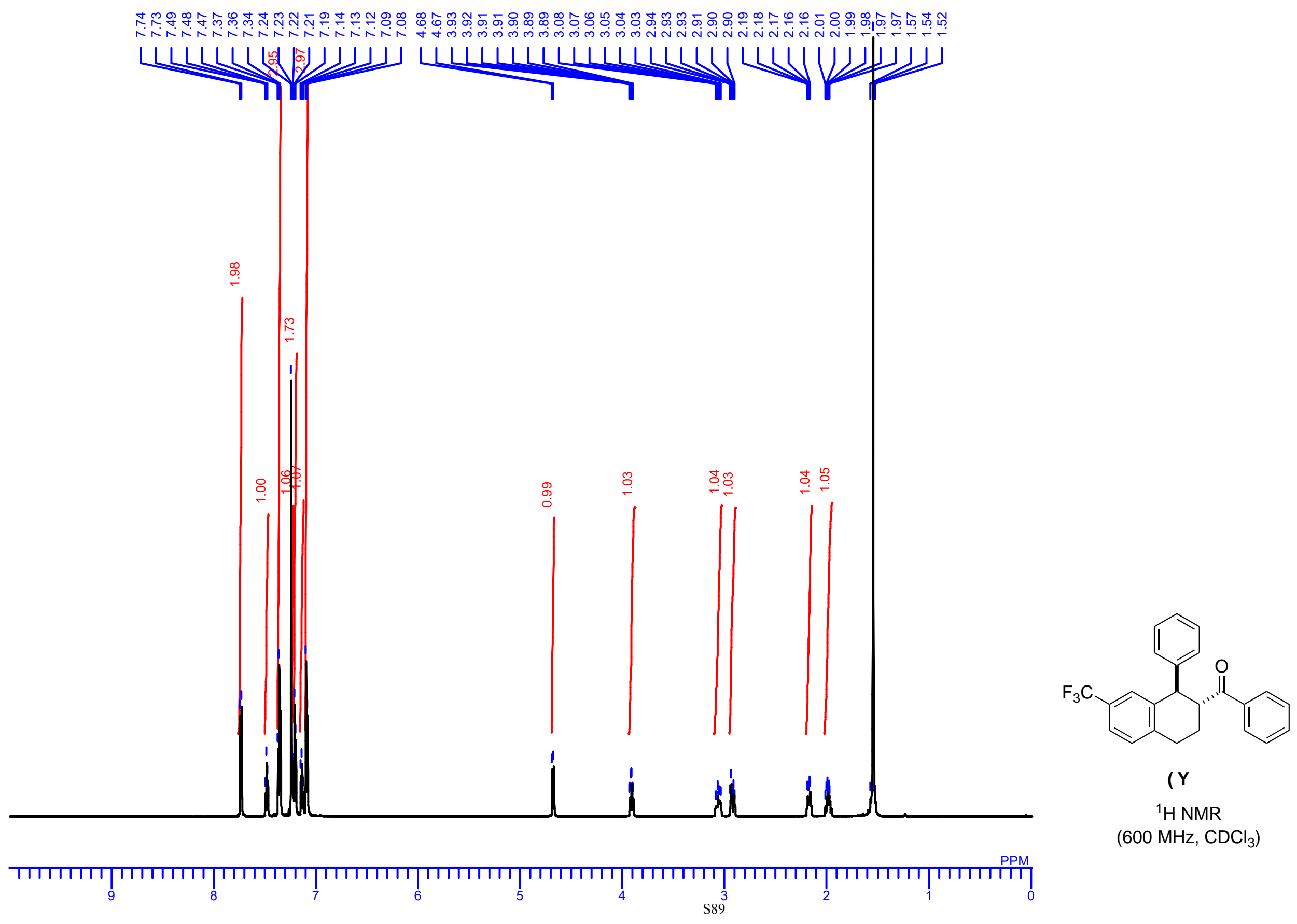




$$
11
$$




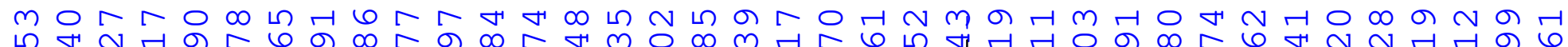
$N N N$ N $N$ -

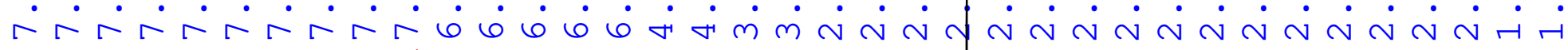

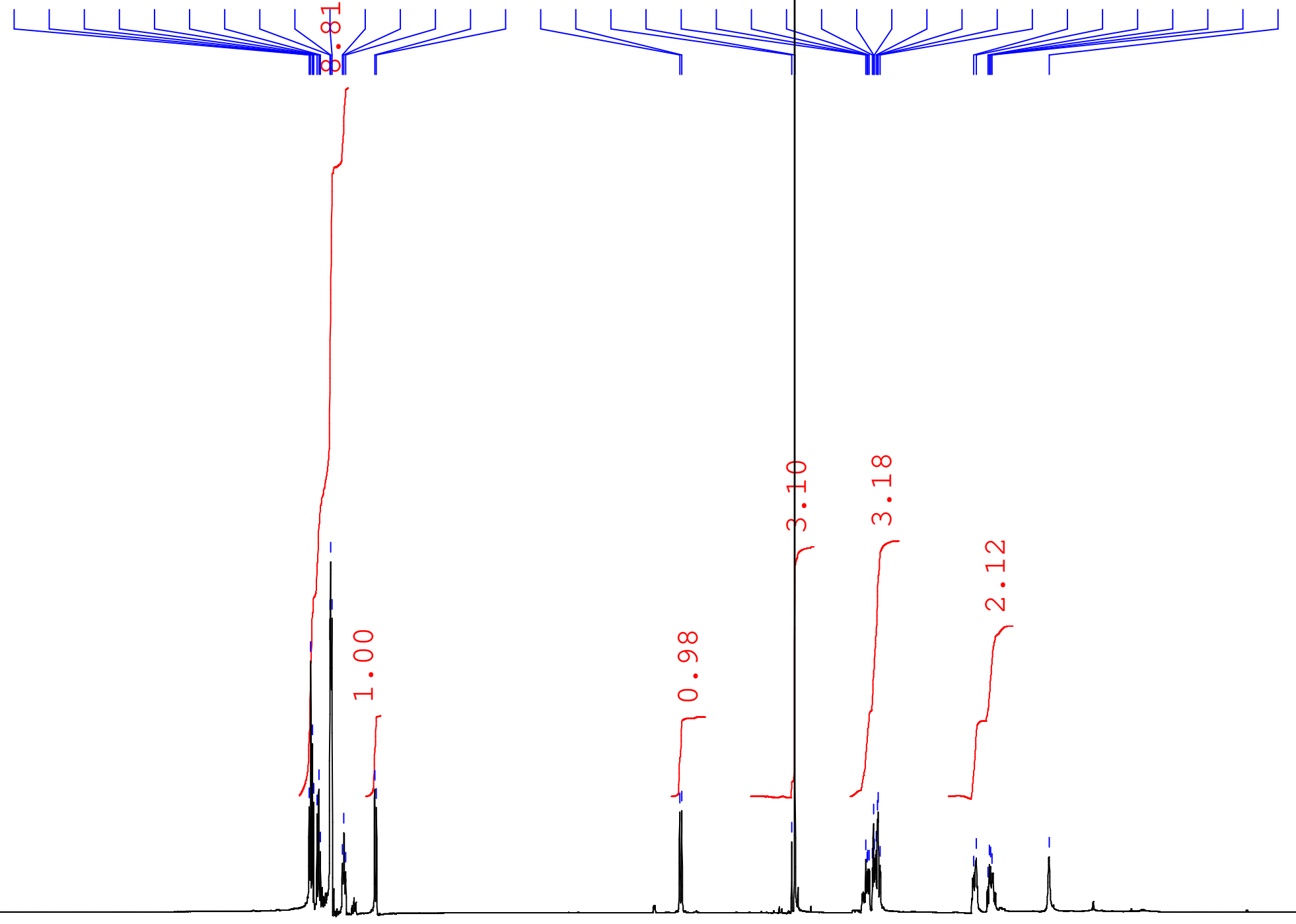



${ }^{1} \mathrm{H}$ NMR $\left(600 \mathrm{MHz}, \mathrm{CDCl}_{3}\right.$ ) 

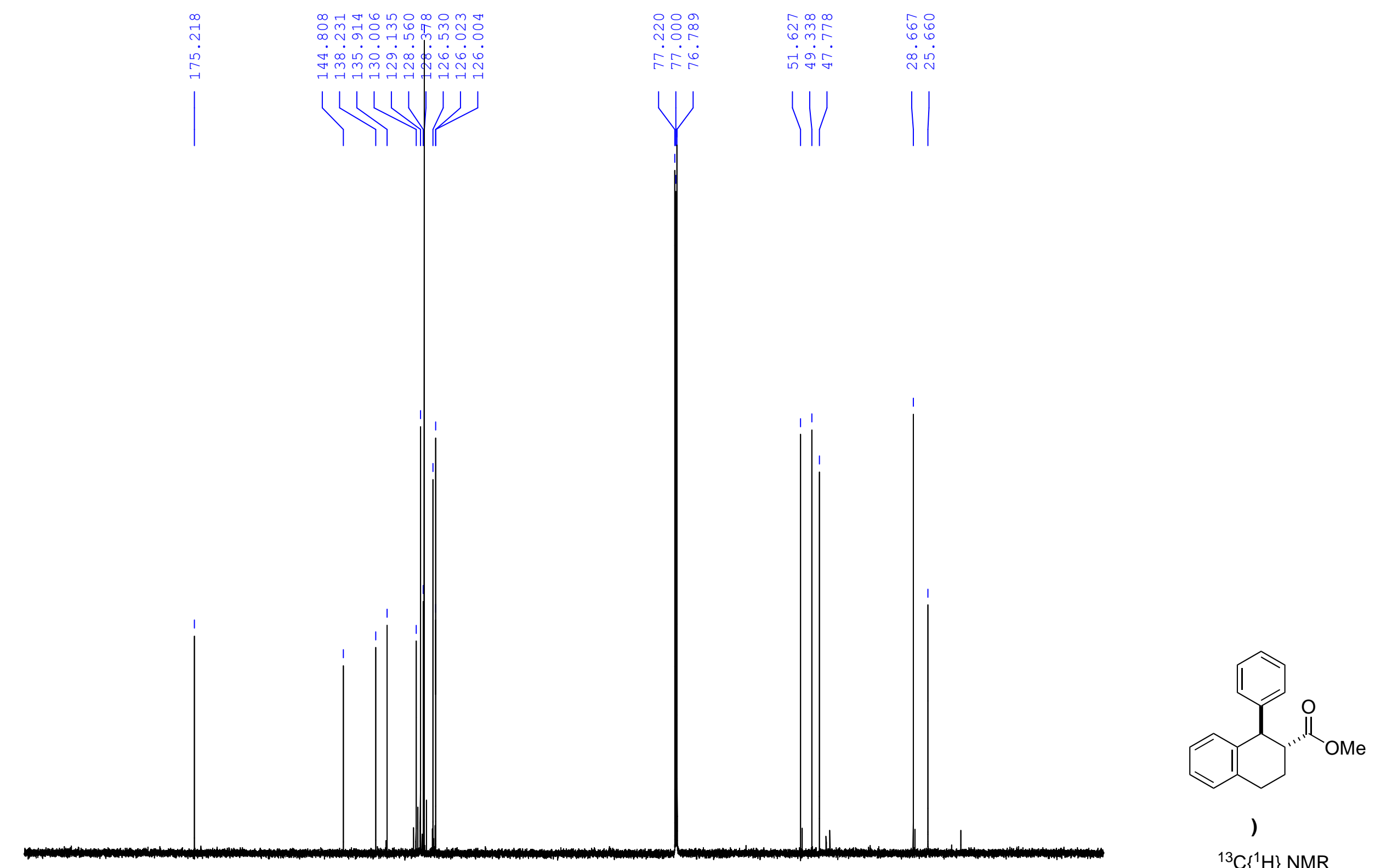

${ }^{13} \mathrm{C}\left\{{ }^{1} \mathrm{H}\right\} \mathrm{NMR}$ $\left(150 \mathrm{MHz}, \mathrm{CDCl}_{3}\right)$

Tा1ा

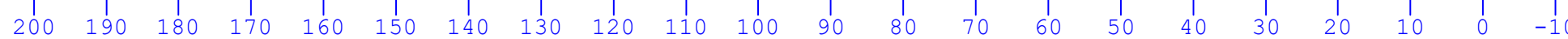





$$
\prod_{10}
$$




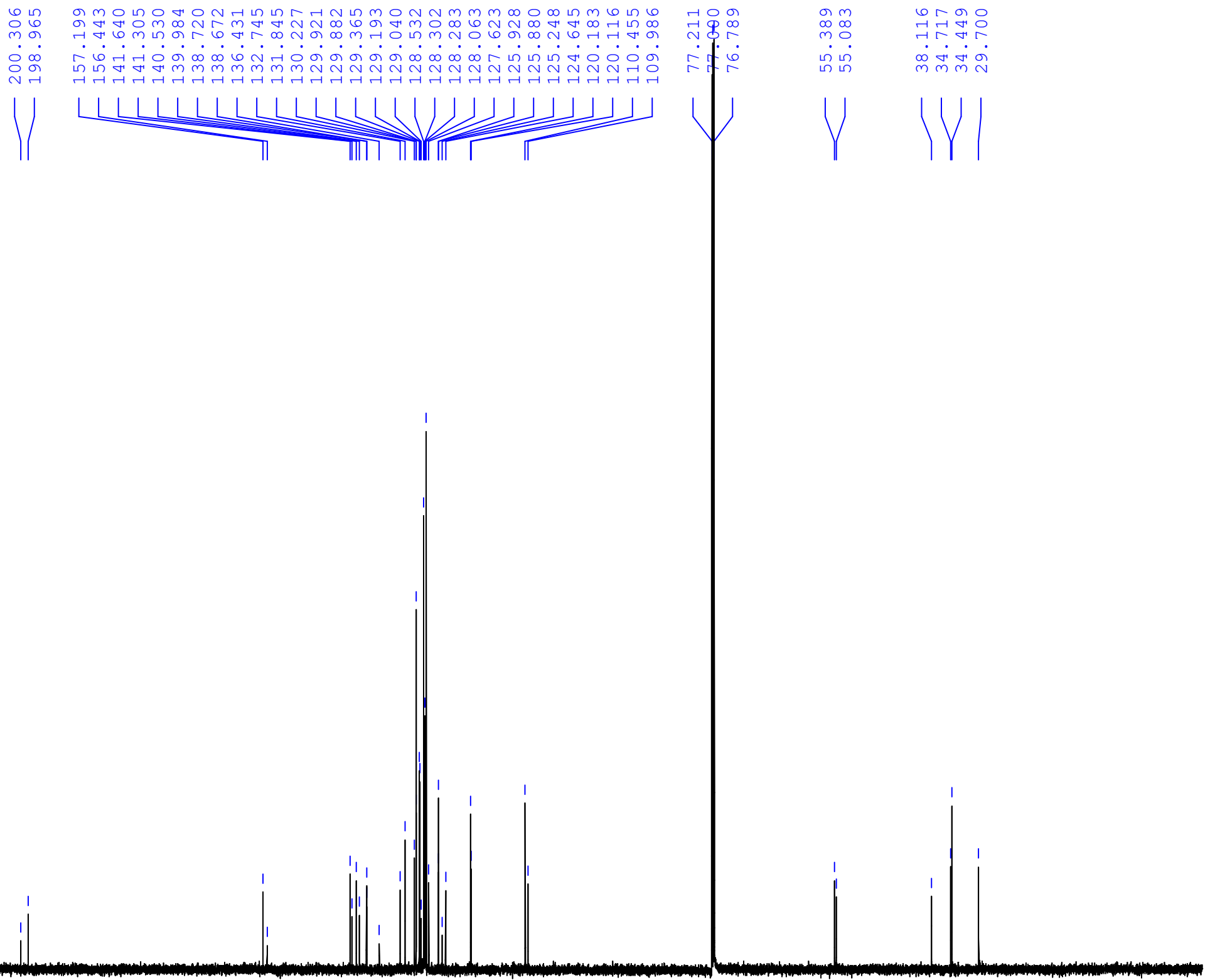



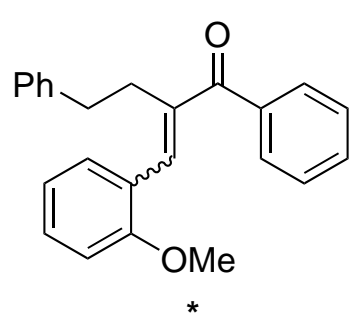

${ }^{13} \mathrm{C}\left\{{ }^{1} \mathrm{H}\right\}$ NMR $\left(150 \mathrm{MHz}, \mathrm{CDCl}_{3}\right.$ ) 


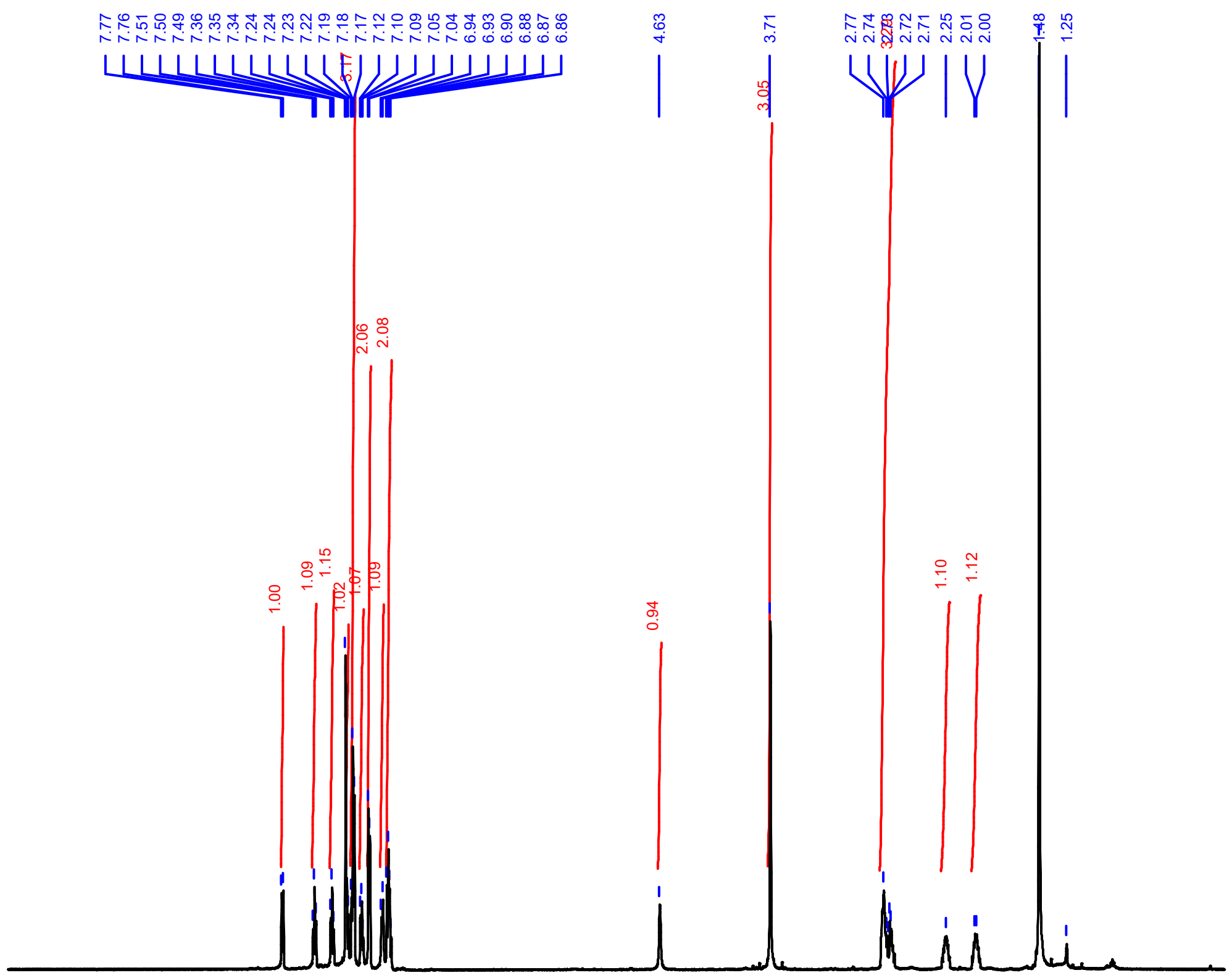

$\prod_{10}$

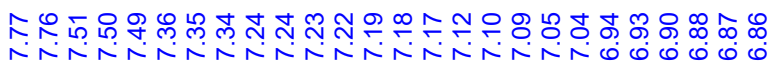

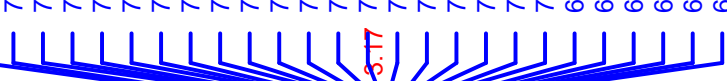
ᄀ 1 III गाााााा $\prod_{9}$
IITाTा
IIIIIII|

7

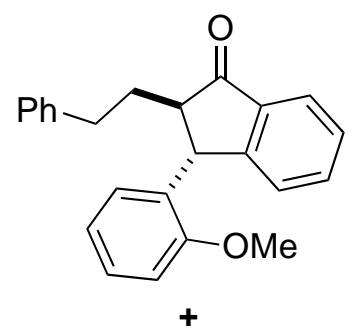

${ }^{1} \mathrm{H}$ NMR (600 MHz, $\mathrm{CDCl}_{3}$ ) 


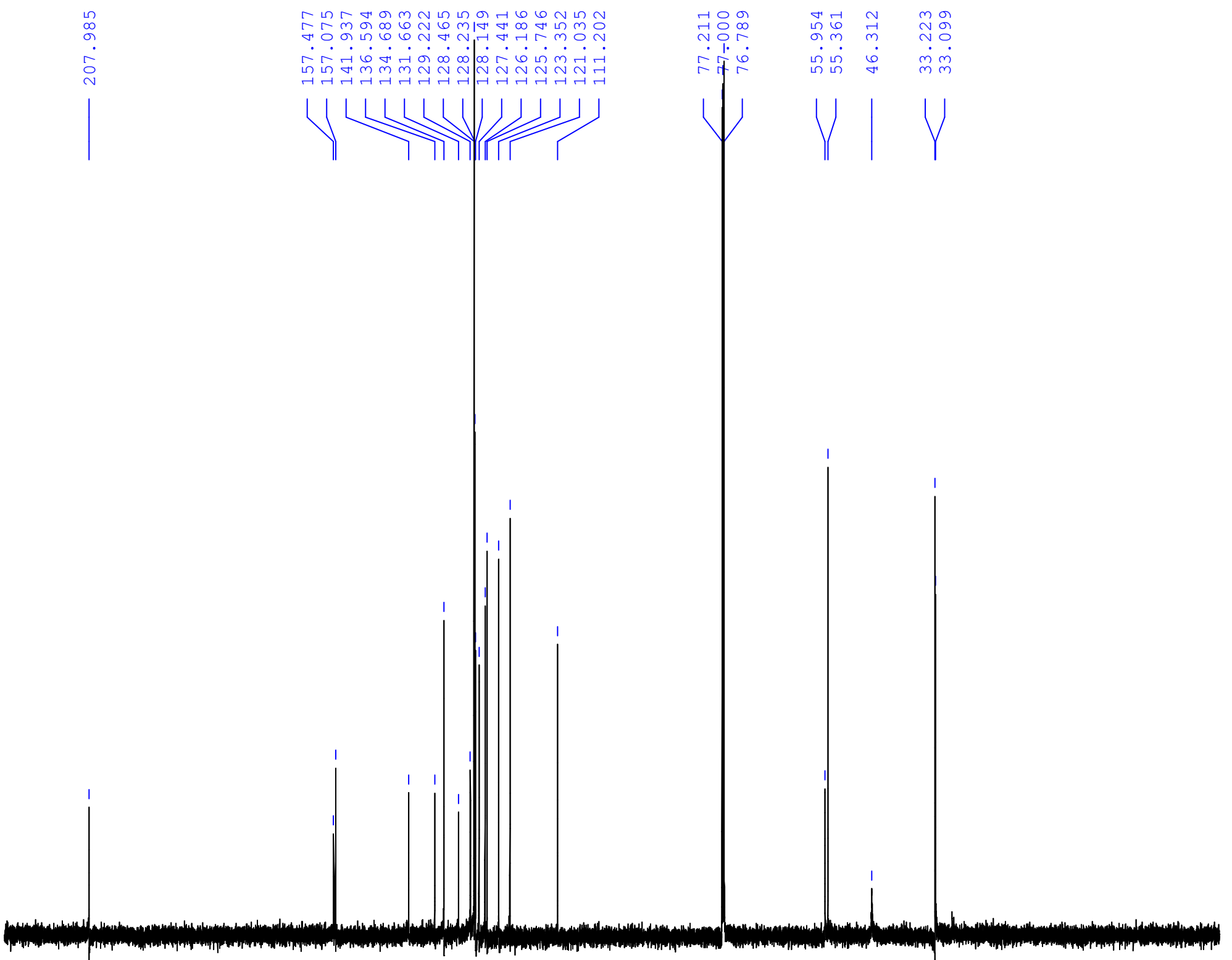

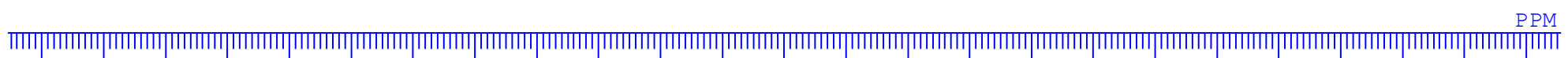

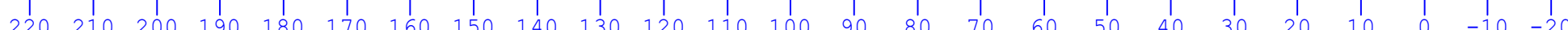

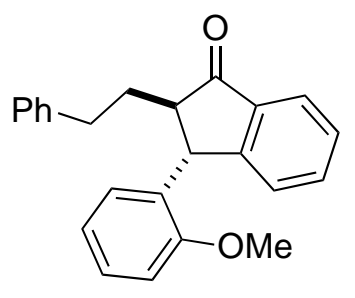

(

${ }^{13} \mathrm{C}\left\{{ }^{1} \mathrm{H}\right\}$ NMR $\left(150 \mathrm{MHz}, \mathrm{CDCl}_{3}\right)$ 